\section{AEC \\ RESEARCH and}

DEVELOPMENT REPORT

\title{
EVALUATION OF RADIOLOGICAL CONDITIONS IN THE VICINITY OF HANFORD FOR 1966 APPENDICES
}

MAY, 1967

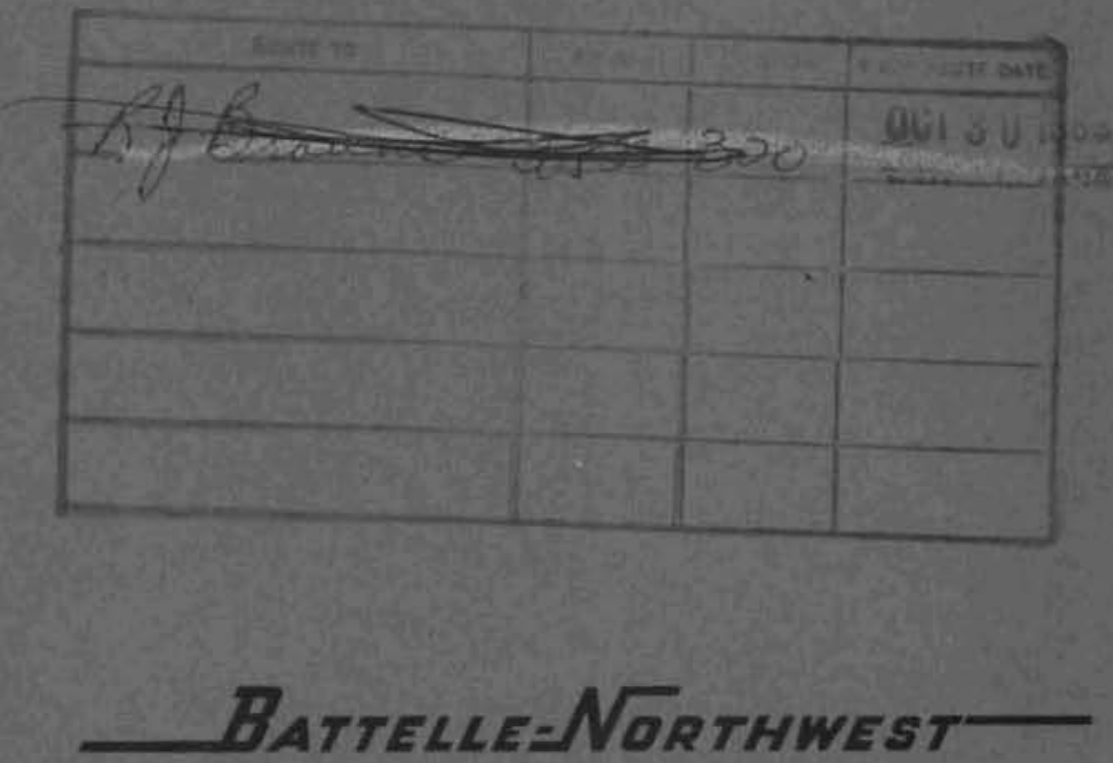

BATTELLE MEMORIAL INSTITUTE / PACIFIC NORTHWEST LABORATORY 


\section{LEGAL NOTICE}

This report was prepared as an account of Government sponsored work. Neither the United States, nor the Commission, nor any person acting on behalf of the Commission:

A. Makes any warranty or representation, expressed or implied, with respect to the accuracy, completeness, or usefulness of the information contained in this report, or that the use of any information, apparatus, method, or process disclosed in this report may not infringe privately owned righis; or

B. Assumes any liabilities with respect to the use of, or for damages resulting from the use of any information, apparatus, method, or process disclosed in this report.

As used in the above, "person acting on behalf of the Commission" includes any employee or contractor of the Commission, or employee of such confractor, to the extent that such employee or contractor of the Commission, or employee of such contractor prepares, disseminates, or provides access to, any information pursuant to his employment or contract with the Commission, or his employment with such contractor.

\section{PACIFIC NORTHWEST LABORATORY \\ RICHLAND, WASHINGTON \\ operated by \\ BATTELLE MEMORIAL INSTITUTE}

for the

UNITED STATES ATOMIC ENERGY COMMISSION UNDER CONTRACT AT(45-1)-1830 


\author{
BNWL-439 APP \\ UC-41 Health and Safety
}

\begin{abstract}
EVALUATION OF RADIOLOGICAL CONDITIONS
IN THE VICINITY OF HANFORD FOR 1966

APPENDICES
\end{abstract}

By

The Environmental Studies Section Staff

J. F. Honstead, Manager

Edited by T. H. Essig

Environmenta1 Hea1th Department

May, 1967

PACIFIC NORTHWEST LABORATORY

RICHLAND, WASHINGTON 
Printed in the United States of America Available from

clearinghouse for Federal Scientific and Technical Information National Bureau of Standards, U.S. Department of Commerce Springfield, Virginia 22151

Price: Printed Copy $\$ 3.00 ;$ Microfiche $\$ 0.65$ 


\section{Preface}

This supplemental report is a compilation of results obtained from both radiochemical analyses of environmental samples and from radiological measurements made in the Hanford environs during 1966. The radiological significance of these data is discussed in the parent report (BNWL-439).

May, 1967

T. H. Essig Environmental Studies Section 
TABLE OF CONTENTS

\section{APPENDIX A \\ RADIONUCLIDES IN COLUMBIA RIVER WATER}

$\underline{\text { Table }}$

1 Concentrations of Radionuclides in Columbia River Water at Vernita Bridge (Grab Samples) Priest Rapids Dam (Cumulative Samples) - 1966

2 Concentrations of Radionuclides in Ciolumbia River Water at Ringold Far Shore (Grab Samples) - 1966

3 Concentrations of Radionuclides in Columbia River Water at Richland (Grab Samples) - 1966

4 Concentrations of Radionuclides in Columbia River Water at Richland (Cumulative Samples) - 1966

5 Cioncentrations of Radionuclides in Columbia River Water at McNary Dam (Cumulative Samples) - 1966

6 Concentrations of Radionuclides in Columbia River Water at Bonneville Dam (Cumulative Samples) - 1966

7 Estimated Rate of Transport of Radionuclides in Columbia River Water at Ringold Far Shore (Grab Samples) - 1966

8 Estimated Rate of Transport of Radionuclides in Columbia River Water at Richland (Grab Samples) - 1966

9 Estimated Rate of Transport of Radionuclides in Columbia River Water at Richland (Cumulative Samples) - 1966

10 Estimated Rate of Transport of Radionuclides in Columbia River Water at McNary Dam (Cumulative Samples) - 1966

11 Estimated Rate of Transport of Radionuclides in Columbia River Water at Bonneville Dam (Cumulative Samples) - 1966 


\section{APPENDIX B \\ RADIONUCLIDES IN DRINKING WATER}

Table

1 Concentrations of Radionuclides in Richland Drinking Water (Grab Samples) - 1966

2 Concentrations of Radionuclides in Rich1and Drinking Water (Cumulative Samples) - 1966

3 Concentrations of Radionuclides in Pasco Drinking Water (Grab Samples) - 1966

4 Concentrations of Radionuclides in Pasco Drinking Water (Cumulative Samples) - 1966

5 Concentrations of Radionuclides in Kennewick Drinking Water (Grab Samples) - 1966

6 Beta Activity in Richland Drinking Water - 1966

7 Beta Activity in Pasco Drinking Water - 1966

8 Beta Activity in Kennewick Drinking Water - 1966

\section{APPENDIX C \\ RADIONUCLIDES IN FISH AND WATERFOWL}

1 Concentrations of Radionuclides in Muscle of Whitefish Taken From the Columbia River - 1966

2 Concentrations of Radionuclides in Muscle of Bass Taken From the Columbia River - 1966

3 Concentrations of Radionuclides in Muscle of Catfish Taken From the Columbia River - 1966

4 Concentrations of Radionuclides in Muscle of Crappie Taken From the Columbia River - 1.966

5 Concentrations of Radionuclides in Muscle of Perch Taken From the Columbia River - 1966 
$\underline{\text { Table }}$

6 Concentrations of Radionuclides in Muscle of Miscellaneous Fish Taken From the Columbia River - 1966

7 Concentrations of Radionuclides in Waterfowl Samples in the Hanford Environs - 1966

\section{APPENDIX D \\ RADIONUCLIDES IN MARINE ORGANISMS}

1 Concentrations of Radionuclides in Oysters From Willapa Bay, Washington - 1966

APPENDIX E

RADIONUCLIDES IN THE ATMOSPHERE

1 Concentrations of ${ }^{13} 1_{I}$ in Air of the Hanford Environs 1966

2 Concentrations of Radioactive Particulates in the Air of the Pacific Northwest - 1966

\section{APPENDIX F \\ RADIONUCLIDES IN FARM PRODUCE \\ AND COMMERCIAL FOODSTUFFS}

1 Concentrations of Radionuclides in Milk - 1966

2 Concentrations of Radionuclides in Hay and Pasture Grass - 1966

3 Concentrations of Radionuclides in Meats Purchased from Local Markets - 1966

4 Concentrations of Radionuclides in Loca1ly Grown Farm Products - 1966

5 Concentrations of Radionuclides in Food Purchased From Local Stores - 1966

\section{APPENDIX G \\ $131_{\text {I IN CATTLE THYROIDS }}$}

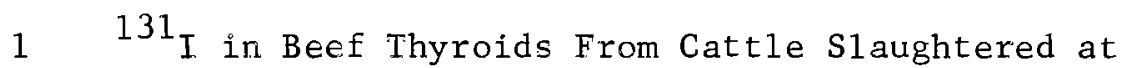
Several Locations - 1.966

\section{APPENDIX H \\ MEASUREMENTS OF EXTERNAL RADIATION EXPOSURE}

1 Ionization Chamber Measurements for the Hanford Reservation and Richland - 1966

2 Ionization Chamber Measurements in the Columbia River - 1966 
APPENDIX A

RADIONUCLIDES IN COLUMBIA RIVER WATER 
APPENDIX A

TABLE 1

CONCENTRATIONS OF RADIONUCLIDES IN COLUMBIA RIVER WATER AT VERNITA BRIDGE (GRAB SAMPLES) /PRIEST RAPIDS DAM (CUMULATIVE SAMPLES) 1966

Units of $\mathrm{pCi} / 1$ of water

\section{VERNITA}

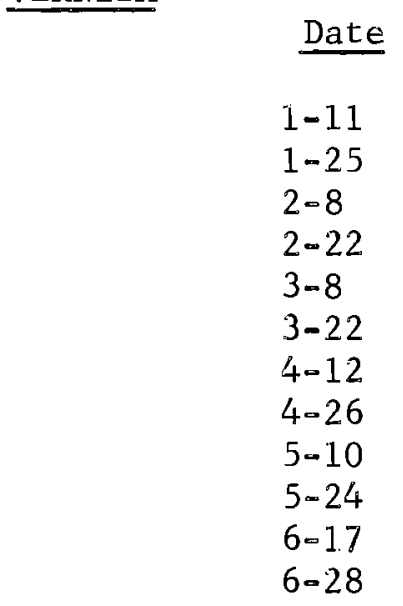

Total

Alpha

0.43

0.20

0.33

0.09

0.61

0.55

$<1.00$

$<1.00$

1.12

$<1.00$

$<1.00$

0.43

PRIESTS RAPIDS

\section{$6-28$}

$7-5$

$7-12$

$7-19$

$7-26$

$8=2$

$8-10$

$8-16$

$8=23$

8-30

$9-7$

$9-13$

9.20

$9-27$

$10-4 \quad 10-11$

$10-11 \quad 10-18$

$1.0-18 \quad 10-25$

10-25 11-1

11-1 11-8

$11-8 \quad 11-15$

$11-15 \quad 11-22$

$11-22$ 11-29

11-29 12-6

$12-6 \quad 12-13$

$12-13 \quad 12-20$

$12-20 \quad 12-28$
$<1.0$

$<1.0$

$<1.0$

$<1.0$

$<1.0$

$<1.0$

$<1.0$

$<1.0$

$<1.0$

$<1.0$

$<1.0$

1.1

$<1.0$

$<1.0$

$<1.0$

$<1.0$

$<1.0$

$<1.0$

$<1.0$

$<1.0$

$<1.0$

$<1.0$

$<1.0$

$<1.0$

$<1.0$

1.26
3

$90 \mathrm{Sr}$

0.98

1.05

1.11

0.89

0.82

LOST

0.83

0.81

0.76

0.67

0.78

0.86

0.65

0.86

0.55

0.54

$<0.50$

0.54

0.60

0.68

0.87

0.74

0.72

0.54

0.79

0.85

0.74

0.63

0.64

0.63

0.90

1.19

0.85

1.03

0.92

0.53

0.80

0.91

No entry indicates no analysis made. 


\section{APPENDIX A}

TABLE 2

CONCENTRATIONS OF RADIONLCLTDES IN

COLIMBIA RIVER WATER AT RINGOLD FAR SHORE (Grab Samples) - 1966

\begin{tabular}{|c|c|c|c|c|c|c|c|c|c|c|}
\hline & & & & & & $0 t$ & $1 / 1$ & & & \\
\hline Date & $\mathrm{RE}+\mathrm{Y}$ & ol & $3 e_{F}$ & 51. & $5 \varepsilon_{\mathrm{Mn}}$ & $64 \mathrm{Cu}$ & $69 \mathrm{~m}$ & 70 & $\begin{array}{l}76 \\
\mathrm{H}\end{array}$ & $\begin{array}{l}239 \\
\mathrm{~Np}\end{array}$ \\
\hline 1.25 & 350 & 4100 & 180 & 3900 & 940 & 1700 & $<70$ & $\overline{3} 20$ & 610 & 290 \\
\hline $2-22$ & 320 & 4900 & 86 & 3800 & 1600 & 2600 & 0 & 510 & 520 & 650 \\
\hline $3=22$ & 670 & 4100 & 190 & 3300 & 2700 & 2500 & 140 & 260 & 450 & 540 \\
\hline $4-26$ & 380 & 3200 & 140 & 2900 & 8900 & 2000 & $<70$ & 370 & 340 & 580 \\
\hline $5=24$ & 330 & 1700 & 91 & 1500 & 2000 & 1400 & $<70$ & 160 & 250 & 420 \\
\hline $6-28$ & 230 & 830 & 34 & 900 & 820 & 760 & $<70$ & $<75$ & 89 & 280 \\
\hline $7-27$ & $<5$ & $<35$ & $<6$ & $<70$ & $<50$ & $<20$ & $<70$ & $<70$ & $<5$ & $<10$ \\
\hline $8-23$ & $<5$ & $<35$ & $<6$ & $<70$ & $<50$ & $<70$ & $<70$ & 87 & $<5$ & $<10$ \\
\hline $9-27$ & 68 & 3000 & 280 & 2500 & 720 & 2.100 & 73 & 350 & 370 & 590 \\
\hline $10-26$ & 110 & 4000 & 200 & 2900 & 880 & 2500 & 210 & 620 & 420 & 690 \\
\hline $11-22$ & 180 & 2700 & 48 & 1600 & 540 & 1700 & 79 & 210 & 270 & 410 \\
\hline $12-27$ & 400 & 4200 & 220 & 4900 & 810 & 4000 & 290 & 540 & 540 & 1100 \\
\hline
\end{tabular}


APPENDIX A

TABLE 3

CONCENTRATIONS OF RADTONUCLIDES IN

COLIJMBIA RIVER WATER AT RICHLAND (Grab Samples) - 1966

\begin{tabular}{|c|c|c|c|c|c|c|c|c|c|c|c|}
\hline Date & $R E+Y$ & $3_{\mathrm{H}}$ & 24 & $32_{P}$ & ${ }^{51} \mathrm{Xr}$ & $64 \mathrm{Ju}$ & ${ }^{65} \mathrm{Zn}$ & $\begin{array}{l}76 \\
\mathrm{As}\end{array}$ & $\begin{array}{r}90 \\
\quad 5 x \\
\end{array}$ & $\begin{array}{r}131 \\
\quad \mathrm{~T} \\
\end{array}$ & $\begin{array}{r}239 \\
\quad \quad \mathrm{~Np} \\
\end{array}$ \\
\hline $1-10$ & 230 & 2900 & 2700 & 280 & 8300 & 820 & 69 & 440 & 1.6 & 11 & 480 \\
\hline $2-7$ & 380 & 4700 & 4500 & 370 & 8600 & 1900 & 120 & 730 & 1.2 & 20 & 830 \\
\hline $3-7$ & 730 & 4100 & 5500 & 340 & 7100 & 3000 & 280 & 870 & 1.2 & 12 & 1700 \\
\hline $4-11$ & 430 & 1800 & 3700 & 290 & 4400 & 2100 & 170 & 490 & 0.98 & 7.6 & 1000 \\
\hline $5-9$ & 450 & 1700 & 3300 & 170 & 2800 & 2700 & 180 & 411 & 1.1 & 3.6 & 680 \\
\hline $6-13$ & 260 & $<1000$ & 1100 & 49 & 1000 & 750 & 67 & 140 & 0.76 & 3.2 & 310 \\
\hline $7-11$ & 30 & 1500 & $<35$ & $<6$ & $<70$ & $<48$ & 35 & $<5$ & 0.93 & LOST & $<10$ \\
\hline $8-8$ & $<5$ & $<1000$ & $<35$ & $<6$ & $<70$ & $<20$ & $<20$ & $<5$ & 0.72 & & $<10$ \\
\hline $9-12$ & 120 & 2500 & 1200 & 210 & 5200 & 1000 & 59 & 240 & 0.88 & 3.8 & 730 \\
\hline $10-11$ & 310 & 1400 & 1900 & 160 & 4100 & 1400 & 110 & 900 & 1.0 & 5.6 & 780 \\
\hline $11-7$ & 240 & 2300 & 2800 & 240 & 8300 & 1500 & 340 & 420 & 1.3 & 9.7 & 1400 \\
\hline $12-13$ & 110 & $<1000$ & 4000 & 230 & 8000 & 1800 & 170 & 410 & 0.80 & 5.9 & 1300 \\
\hline
\end{tabular}


APPENDIX A

TABLE 4

CONCENTRATIONS OF RADIONUCLIDES IN

COLUMBIA RIVER WATER AT RICHLAND (Cumulative Samples) - 1966

Date

$12-27-65--1-3$

1-3 $1-10$

$1-10 \quad 1-17$

$1=17 \quad 1-24$

$1-24 \quad 1-31$.

$1-31 \quad 2 \Leftrightarrow 7$

2-7 2-14

$2-14 \quad 2-21$

$2-21 \quad 2-28$

$2=28 \quad 3-7$

$3=7 \quad 3-.14$

$3-14 \quad 3-21$.

$3=21 \quad 3-28$

$3-28 \quad 4-4$

$4-4 \quad 4-11$

$4-11 \quad 4-18$

$4-1.8 \quad 4-25$

$4-25 \quad 5-2$

$5-2 \quad 5-9$

$5=9 \quad 5 \circ 16$

$5-16 \quad 5-23$

$5-23 \quad 5-31$

$5-31 \quad 6-6$

$6-6 \quad 6-13$

$6-13 \quad 6-20$

$6-20 \quad 6-27$

$6-27 \quad 7-5$

$7=5 \quad 7-11$.

$7-11 \quad 7-18$

$7-1.8 \quad 7-25$

$7-25 \quad 8-1$.

8-1 8-8

8-8 8-15

$8=15 \quad 8-22$

$8-22 \quad 8=29$

$8.29 \quad 9-6$

$906 \quad 9-12$

$9-12 \quad 9-19$

$9-19 \quad 9-26$

$9-26 \quad 10-3$
32

180

86
190

200

270

250

100

130

170

180

220

220

250

260

260

190

180

160

180

130

150

58

28

68

35

64

40

$<6.0$

$<6.0$

$<6.0$

$<6.0$

$<6.0$

$<6.0$

26

99

85

1.50

150

160 Units of $\mathrm{pCi} / 1$ of water

$5 i$

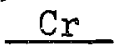

4700

6200

4500

5200

6500

5800

3400

3800

4600

3700

4900

5200

5400

5800

5000

4800

5000

3800

2500

2600

2000

1300

760

1400

1800

1700

850

$<70$

$<70$

$<70$

$<70$

$<70$

$<70$

1400

4400

4300

5000

4400

3400
65

$\mathrm{Zn}$

160

170

150

420

180

200

200

140

180

190

290

260

230

450

340

530

330

420

680

310

360

220

350

180

120

97

140

34

36

$<20$

21

32

$<20$

50

120

110

110

110

160
131

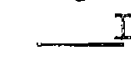

7.2

6.6

6.3

5.6

8.7

11

59 ?

34

12

5.9

6.9

6.5

6.9

6.4

5.1

5.3

4.3

3.4

2.8

3.3

4.0

2.5

2.1

2.0

2.1

3.2

$<2.0$

$<2.0$

$<2.0$

$<2.0$

$<2.0$

$<2.0$

$<2.0$

4.5

6.3

6.4

7.6

6.3

4.9

No entry indicates no analys is made. 
APPENDIX A

TABLE 4 (Continued)

CONCENTRATIONS OF RADIONUCLIDES IN

COLUMBIA RIVER WATER AT RICHLAND (Cumulative Samples) - 1966

\begin{tabular}{|c|c|c|c|c|c|}
\hline & \multicolumn{5}{|c|}{ Units of $\mathrm{pCi} / 1$ of water } \\
\hline & & 32 & 57 & 65 & 131 \\
\hline Date & & $E$ & $\mathrm{Cr}$ & ـ & ... I \\
\hline $10-3$ & $10-10$ & 160 & 3900 & 140 & 8.2 \\
\hline $10-10$ & $10-17$ & 130 & 4300 & 160 & 6.4 \\
\hline $10-17$ & $10-24$ & 130 & 4500 & 120 & 5.8 \\
\hline $10-24$ & $10-31$ & 180 & 4800 & 170 & 5.4 \\
\hline $10-31$ & $11-7$ & 150 & 5400 & 160 & 3.4 \\
\hline $11-7$ & $11-14$ & 160 & 4400 & 120 & 7.3 \\
\hline $11-14$ & $11-21$ & 140 & 4600 & 130 & 5.3 \\
\hline $11-21$ & $11-28$ & 300 & 3200 & 180 & 4.2 \\
\hline $11-28$ & $12-5$ & 120 & 4100 & 170 & 2.7 \\
\hline $12-5$ & $12-12$ & 160 & 4600 & 190 & 5.3 \\
\hline $12-12$ & $12-19$ & 200 & 5500 & 190 & 6.9 \\
\hline $12-19$ & $12-27$ & 200 & 5500 & 170 & 13.5 \\
\hline
\end{tabular}

No entry indicates no analysis made. 


\section{APPENDIX A}

TABLE 5

CONCENTRATIONS OF RADIONUCLIDES IN COLUMBIA RIVER WATER AT MCNARY DAM (Cumulative Samples ) - 1966

\begin{tabular}{|c|c|c|c|c|c|}
\hline \multicolumn{2}{|l|}{ Date } & 32 & 51 & 65 & 131 \\
\hline $12-30$ & $65--1-7$ & 240 & 2500 & 84 & 3.3 \\
\hline $1-7$ & $1-14$ & 100 & 3300 & 99 & 5.7 \\
\hline $1-14$ & $1-21$ & 96 & 3000 & 91 & \\
\hline $1-21$ & $1-28$ & 91 & 2800 & 92 & 3.6 \\
\hline 1.28 & $2-4$ & 710 & 3200 & 93 & 3.5 \\
\hline $2-4$ & $2-10$ & 240 & 3400 & 120 & 3.8 \\
\hline $2-10$ & $2-18$ & 100 & 2200 & 120 & 170 \\
\hline $2-18$ & $2-25$ & 55 & 1800 & 79 & 43 \\
\hline $2-25$ & $3-4$ & 66 & 2700 & 120 & 7.2 \\
\hline $3-4$ & $3-11$ & 97 & 2100 & 110 & 3.6 \\
\hline $3-11$ & $3-25$ & 93 & 2400 & 93 & 2.3 \\
\hline $3-25$ & $4-1$ & 110 & 2900 & 100 & 13.0 \\
\hline $4-1$ & $4-8$ & 100 & 2200 & 83 & $<2.2$ \\
\hline $4-8$ & $4-15$ & 1.00 & 2100 & 74 & 2.1 \\
\hline $4-15$ & $4-22$ & 67 & 1800 & 53 & \\
\hline $4-22$ & $4-29$ & 62 & 1800 & 83 & $<2.0$ \\
\hline $4-29$ & $5-6$ & 130 & 1900 & 67 & 2.2 \\
\hline $5-6$ & $5-13$ & 65 & 1400 & 61 & $<2.0$ \\
\hline $5-13$ & $5-20$ & 51 & 1200 & 73 & $<2.0$ \\
\hline $5-20$ & $5-27$ & 45 & 1300 & 67 & $<2.0$ \\
\hline $5-27$ & $6-3$ & 52 & 1000 & 74 & $<2.0$ \\
\hline 6.3 & $6 \cdot 10$ & 34 & 730 & 73 & $<2.0$ \\
\hline $6-10$ & $6-17$ & 33 & 540 & 92 & $<2.0$ \\
\hline $6-17$ & $6-24$ & 32 & 1200 & 72 & $<2.0$ \\
\hline $6-24$ & $7-1$ & 41. & 1100 & 47 & $<2.0$ \\
\hline $7-1$ & $7-8$ & 41 & 1100 & 46 & $<2.0$ \\
\hline $7-8$ & $7=15$ & 23 & 300 & 34 & $<2.0$ \\
\hline $7-15$ & $7-22$ & $<6.0$ & $<70$ & 28 & $<2.0$ \\
\hline $7-22$ & $7-29$ & $<6.0$ & $<70$ & $<20$ & $<2.0$ \\
\hline $7-29$ & $8-5$ & $\angle 6.0$ & $<70$ & $<20$ & $<2.0$ \\
\hline $8-5$ & $8-12$ & $\angle 6.0$ & $<70$ & $<20$ & $<2.0$ \\
\hline $8-12$ & $8-19$ & $<6,0$ & $\angle 70$ & $<20$ & $<2.0$ \\
\hline $8-19$ & $8=26$ & $<6,0$ & 82 & 21 & $<2.0$ \\
\hline $8-26$ & $9-2$ & $<6.0$ & $<110$ & 21 & 2.5 \\
\hline $9-2$ & 9.9 & 31 & 2600 & $<20$ & 2.4 \\
\hline $9-9$ & $9-16$ & 33 & 2500 & 23 & $<2,0$ \\
\hline $9-16$ & 9.23 & 50 & 2700 & 20 & 3.1 \\
\hline $9-23$ & $9-30$ & 48 & 2500 & 20 & 2.2 \\
\hline $9-30$ & $10-7$ & 63 & 1900 & $<20$ & 2.1 \\
\hline $10-7$ & $10-14$ & 47 & 1900 & 32 & 2.6 \\
\hline
\end{tabular}

No entry indicates no analys is made. 
APPENDIX A

TABLE 5 (Continued)

CONCENTRATIONS OF RADIONUCLIDES IN COLUMBIA RIVER WATER AT MCNARY DAM (Cumulative Samples) - 1966 Units of $\mathrm{pCi} / 1$ of water

$\underline{\text { Date }}$

!.

\section{$10-14 \quad 10-21$}

$10-21 \quad 10-28$

$10-28 \quad 11-4$

$11-4 \quad 11-11$

$11-11 \quad 11-18$

$11-18 \quad 11-23$

$11-23 \quad 12-2$

$12-2 \quad 12-9$

$12-9 \quad 12-16$

$12-16 \quad 12-22$

$12-22 \quad 12-30$

\begin{abstract}
32
\end{abstract}
P

47

98

62

61

55

52

67

41

61

80

72
51

$\mathrm{Cr}$

2800

2600

2300

2800

2400

3000

1800

1800

2200

3000

3000

\section{5}

$\mathrm{Zn}$

36

44

40

54

47

49

60

44

69

89

92
131.

I

2.7

3.2

$<2.0$

2.1

4.4

2.4

2.9

$<2.0$

$\angle 2.0$

2.7

6.4

No entry indicates no analys is made. 


\section{APPENDIX A}

TABLE 6

CONCENIRATIONS OF RADIONUCLIDES IN COLUMBIA

RIVER WATER AT BONNEVILLE DAM (Cumulative Samples) - 1966

\begin{tabular}{|c|c|c|c|c|c|}
\hline \multicolumn{2}{|l|}{ Date } & $\begin{array}{r}32 \\
\end{array}$ & $\begin{array}{c}51 \\
\mathrm{Cx} \\
\end{array}$ & $\begin{array}{l}65 \\
\mathrm{Zn} \\
\end{array}$ & $\begin{array}{r}131 \\
I\end{array}$ \\
\hline \multicolumn{2}{|c|}{$12-28-65-1-4$} & 36 & 2000 & 30 & 2.3 \\
\hline $1-4$ & $1-11$ & 33 & 2000 & 57 & 1.7 \\
\hline $1-11$ & $1-25$ & 33 & 1600 & 29 & 1.5 \\
\hline $1-25$ & $2-8$ & 39 & 2000 & 34 & 3.8 \\
\hline $2-8$ & $2-22$ & 23 & 1900 & 61 & 8.2 \\
\hline $2-22$ & $3-8$ & 40 & 1500 & 26 & 24 \\
\hline $3=8$ & $3-22$ & 34 & 1600 & 66 & 2.3 \\
\hline $3-22$ & $4-5$ & 45 & 1700 & 70 & $<2.0$ \\
\hline $4-5$ & $4-19$ & 44 & 1600 & 64 & 2.0 \\
\hline $4 \infty 19$ & $5-3$ & 36 & 1400 & 54 & $<2.0$ \\
\hline $5-3$ & $5 \times 17$ & 39 & 1300 & 120 & $<2.0$ \\
\hline $5 m 17$ & $5-31$ & 37 & 1.100 & 100 & $<2.0$ \\
\hline $5-31$ & $6 \cdot 14$ & 28 & 800 & 160 & $<2.0$ \\
\hline $6-14$ & $6-28$ & 25 & 1200 & 83 & $<2.0$ \\
\hline $6 \cdot 28$ & $7-12$ & 27 & 92.0 & 73 & $<2.0$ \\
\hline $7-12$ & $7-19$ & & 280 & 69 & $<2.0$ \\
\hline $7-19$ & $7-26$ & $<6.0$ & $<70$ & 34 & $<2.0$ \\
\hline $7-26$ & $8-2$ & $<6.0$ & $<70$ & $<20$ & $<2.0$ \\
\hline $8-2$ & 8.16 & $<6.0$ & $<70$ & 33 & $<2.0$ \\
\hline $8 \infty 16$ & $8-23$ & $<6.0$ & $<70$ & 24 & $<2.0$ \\
\hline $8-23$ & $8-30$ & $<6.0$ & $<70$ & 30 & $<2.0$ \\
\hline $8-30$ & $9-6$ & $<6.0$ & $<70$ & $<20$ & $<2.0$ \\
\hline $9=6$ & $9-13$ & $<6.3$ & 890 & $<20$ & 2.7 \\
\hline $9=0.13$ & $9-20$ & 11 & 1700 & $<20$ & 5.0 \\
\hline $9-20$ & $9-27$ & 14 & 1700 & $<20$ & $<2.0$ \\
\hline $9-27$ & $10-4$ & 15 & 1800 & $<20$ & 2.0 \\
\hline $10-4$ & $10-18$ & 20 & 1300 & $<20$ & 2.0 \\
\hline $10-18$ & $10-25$ & 18 & 1500 & $<20$ & $<2.0$ \\
\hline $10-25$ & $11=1$ & 26 & 2000 & $<20$ & $<2.0$ \\
\hline $11-1$ & $1.1-8$ & 18 & 1800 & $<20$ & $<2.0$ \\
\hline $11=8$ & $11-15$ & 21 & 1700 & $<20$ & $<2.0$ \\
\hline $11-15$ & $11-22$ & 38 & 1700 & $<20$ & $<2.0$ \\
\hline $11-22$ & $11-29$ & 25 & 1900 & $<20$ & 6.4 \\
\hline $11-29$ & $1.2-6$ & 23 & 1300 & $<20$ & $<2.0$ \\
\hline $12-6$ & $12-13$ & 11 & 1200 & $<20$ & $<2.0$ \\
\hline $12-1.3$ & $12-20$ & 22 & 1200 & $<20$ & 3.2 \\
\hline $12-20$ & $12-27$ & 23 & 1800 & 28 & $<2.0$ \\
\hline
\end{tabular}

No entry indicates no analysis made. 


\begin{tabular}{|c|c|c|c|c|c|c|c|c|c|c|}
\hline & & & & & 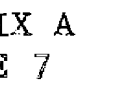 & & & & & \\
\hline & & & $\begin{array}{l}\text { TMATI } \\
\text { IA R }\end{array}$ & $\begin{array}{l}\text { CE OF } \\
\text { VATER }\end{array}$ & $\begin{array}{l}\text { PORT O } \\
\text { NGOLD }\end{array}$ & $\begin{array}{l}\text { LONUGLI I } \\
\text { HORE (G }\end{array}$ & Samples & 1966 & & \\
\hline Date & $\underline{\mathrm{RE}+\mathrm{Y}}$ & $\begin{array}{r}24 \\
\quad \mathrm{Ng} \\
\end{array}$ & $\begin{array}{r}32 \\
E \\
\end{array}$ & $\begin{array}{l}51 . \mathrm{U} \\
\mathrm{Cr} \\
\end{array}$ & $\begin{array}{c}f \text { cur } \\
56 \\
\mathrm{Mnn}\end{array}$ & $\begin{array}{l}64 \\
\mathrm{Cu} \\
\end{array}$ & $\begin{array}{r}60 \mathrm{~m} \\
-\quad \mathrm{mn}\end{array}$ & $\begin{array}{l}72 \\
\quad \mathrm{Ga} \\
\end{array}$ & $\begin{array}{l}76 \\
\mathrm{As} \\
\end{array}$ & $\begin{array}{r}39 \\
\quad \quad N p \\
\end{array}$ \\
\hline $1-25$ & 69 & 810 & 36 & 700 & 190 & 330 & $<14$ & 65 & 120 & 57 \\
\hline $2-22$ & 63 & 960 & 17 & 760 & 320 & 520 & 0 & 100 & 100 & 130 \\
\hline $3-22$ & 130 & 790 & 36 & 640 & 520 & 470 & 27 & 50 & 86 & 100 \\
\hline $4-26$ & 100 & 830 & 38 & 750 & 2300 & 530 & $<18$ & 97 & 89 & 150 \\
\hline $5-24$ & 170 & 870 & 46 & 870 & 950 & 730 & $<35$ & 79 & 120 & 21.0 \\
\hline $6-28$ & 140 & 510 & 21 & 550 & 500 & 460 & $<42$ & 46 & 54 & 170 \\
\hline $7-27$ & $<3$ & $<18$ & $\angle 3$ & $<36$ & $\angle 25$ & -10 & $<36$ & $<36$ & $<3$ & $<5$ \\
\hline $8-23$ & $<1$ & $<8$ & $<1$ & $<15$ & $<11$ & $\therefore 15$ & $<15$ & $<19$ & $<1$ & $<2$ \\
\hline $9-27$ & 14 & 610 & 57 & 520 & 150 & 430 & 15 & 73 & 77 & 120 \\
\hline $10-26$ & 20 & 710 & 36 & 510 & 160 & 440 & 37 & 110 & 75 & 120 \\
\hline $11-22$ & 40 & 620 & 11 & 360 & 120 & 380 & 18 & 48 & 61 & 93 \\
\hline $12-27$ & 100 & 1100 & 56 & 1300 & 210 & 1000 & 74 & 140 & 140 & 290 \\
\hline
\end{tabular}

No entry indicates no analysis made. 


\section{APPENDIX A}

TABLE 8

ESTIMATED RATE OF TRANSPORT OF RADTONUCLIDES

IN COLUMBIA RIVER. WATER AT RICHLAND (Grab Samples) - 1966

\begin{tabular}{|c|c|c|c|c|c|c|c|c|c|c|}
\hline Date & $\underline{R E}+\underline{Y}$ & $\begin{array}{l}24 \\
\mathrm{Ng} \\
\end{array}$ & $\begin{array}{r}32 \\
E \\
\end{array}$ & $\begin{array}{l}51 \\
\mathrm{Cr} \\
\end{array}$ & $\begin{array}{l}\text { of Cur } \\
56 \\
\mathrm{Mn}\end{array}$ & $\begin{array}{l}\text { Day } \\
64 \\
61 \\
\end{array}$ & $\begin{array}{r}69 \mathrm{~m} \\
\mathrm{Zn} \\
\end{array}$ & $\begin{array}{r}72 \\
\quad \mathrm{Ga} \\
\end{array}$ & $\begin{array}{l}76 \\
\mathrm{As} \\
\end{array}$ & $\begin{array}{r}239 \\
\quad \mathrm{~Np} \\
\end{array}$ \\
\hline $1-10$ & 43 & 510 & 53 & 1600 & 150 & 13 & 83 & 0.31 & 2.0 & 90 \\
\hline $2-7$ & 82 & 990 & 81 & 1900 & 410 & 25 & 160 & 0.26 & 4.4 & 180 \\
\hline $3-7$ & 170 & 1300 & 80 & 1700 & 680 & 65 & 200 & 0.28 & 2.8 & 410 \\
\hline $4-11$ & 100 & 870 & 68 & 1000 & 500 & 41 & 120 & 0.23 & 1.8 & 240 \\
\hline $5-9$ & 220 & 1600 & 85 & 1400 & 1300 & 88 & 200 & 0.52 & 1.8 & 330 \\
\hline $6-13$ & 200 & 860 & 37 & 790 & 570 & 51 & 110 & 0.58 & 2.4 & 230 \\
\hline $7-11$ & $<18$ & $<21$ & $<4$ & $<42$ & $<29$ & 21 & $<3$ & 0.56 & LOST & $<6$ \\
\hline $8-8$ & $<2$ & $<12$ & $<2$ & $<23$ & $<7$ & $<7$ & $<2$ & 0.24 & & $<3$ \\
\hline $9-12$ & 22 & 230 & 38 & 970 & 180 & 11 & 44 & 0.16 & 0.70 & 140 \\
\hline $10-11$ & 62 & 390 & 33 & 830 & 21 & 21 & 180 & 0.21 & 1.1 & 160 \\
\hline $11-7$ & 45 & 530 & 44 & 1500 & 290 & 63 & 77 & 0.25 & 1.8 & 270 \\
\hline $12-13$ & 22 & 820 & 46 & 1600 & 360 & 35 & 82 & 0.16 & 1.2 & 260 \\
\hline
\end{tabular}

No entry indicates no analysis made. 


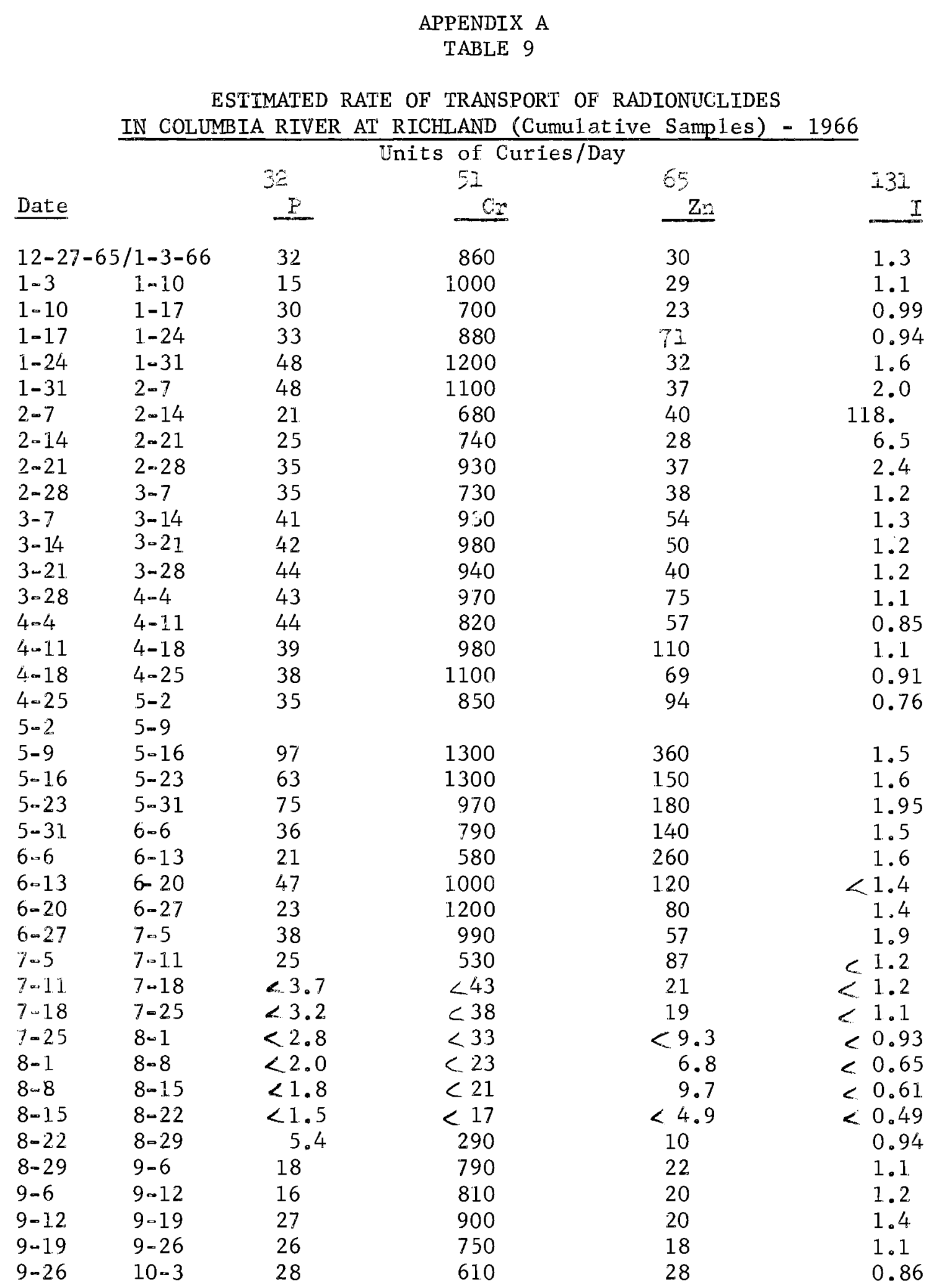

No entry indicates no analysis made. 


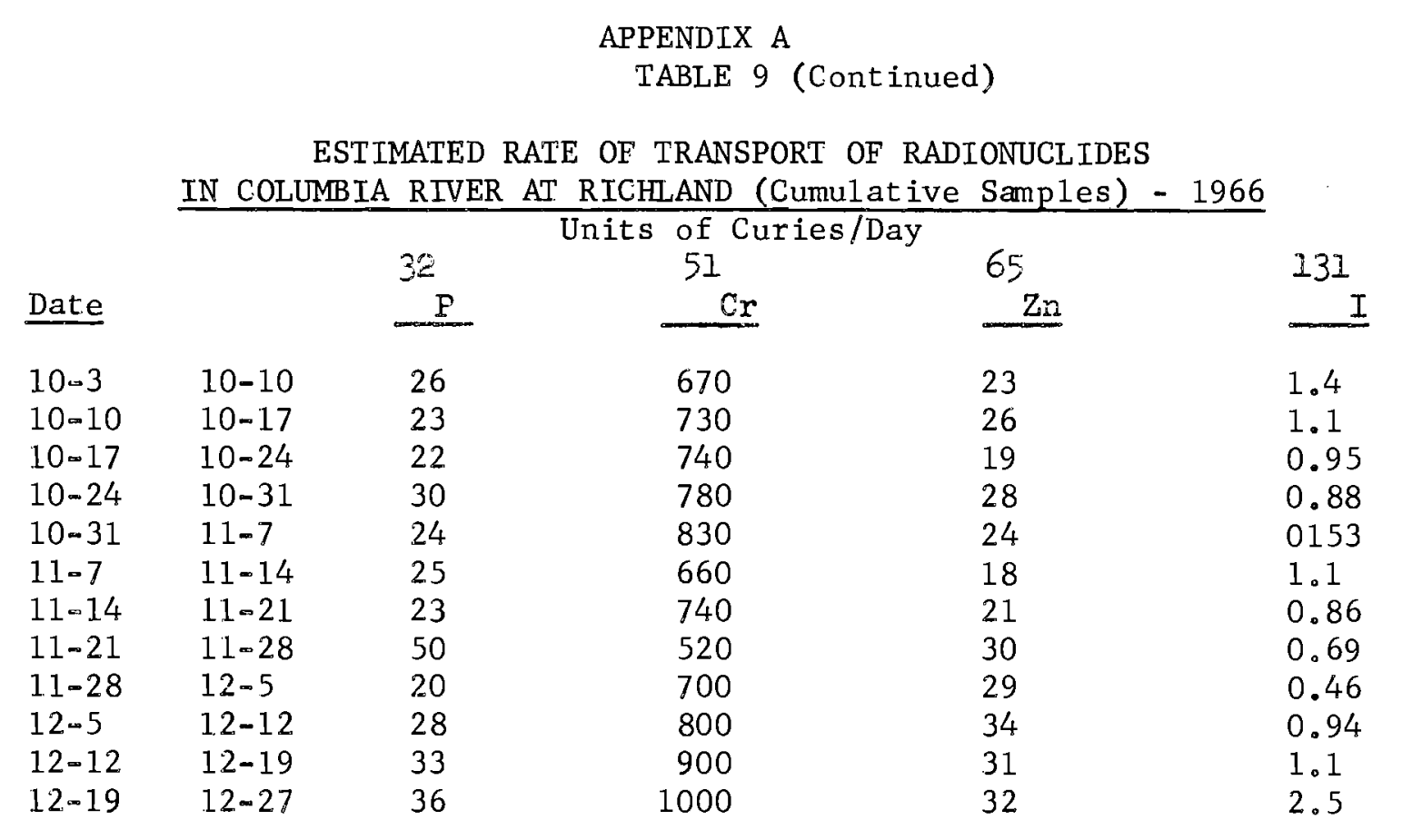

No entry indicates no analys is made. 


\section{APPENDIX A \\ TABLE 10}

ESTIMATED RATE OF TRANSPORT OF RADIONLCLIDES

IN COLUMBIA RIVER WATER AT MCNARY DAM (Cumulative Samples) - 1966

\begin{tabular}{|c|c|c|c|c|c|}
\hline Date & & $\begin{array}{r}32 \\
\quad \mathrm{P} \\
\end{array}$ & $\begin{array}{r}\text { of Cur } \\
51 \\
\mathrm{Cr} \\
\end{array}$ & $\begin{array}{l}65 \\
\mathrm{Zn} \\
\end{array}$ & 231 \\
\hline $12-30$ & $/ 1-7-66$ & 64 & 680 & 22 & 0.88 \\
\hline 1.7 & $1-14$ & 27 & 880 & 26 & 1.5 \\
\hline $1-14$ & $1-21$ & 25 & 790 & 24 & \\
\hline $1-21$ & $1-28$ & 25 & 750 & 25 & 0.98 \\
\hline $1=28$ & $2-4$ & 210 & 940 & 27 & 1.0 \\
\hline $2-4$ & $2-10$ & 68 & 940 & 34 & 1.1 \\
\hline $2-10$ & $2-18$ & 29 & 640 & 33 & 48 \\
\hline $2-18$ & $2-25$ & 15 & 470 & 21 & 11 \\
\hline $2-25$ & $3-4$ & 21 & 880 & 38 & 2.3 \\
\hline $3-4$ & $3-11$ & 27 & 570 & 31 & 0.99 \\
\hline $3-11$ & $3-25$ & 28 & 740 & 28 & 0.70 \\
\hline $3-25$ & $4-1$ & 34 & 930 & 32 & 4.1 \\
\hline $4-1$ & $4-8$ & 37 & 800 & 30 & 0.79 \\
\hline $4-8$ & $4-15$ & 38 & 760 & 27 & 0.77 \\
\hline $4-15$ & $4-22$ & 21 & 580 & 17 & \\
\hline $4-22$ & $4-29$ & 2.1 & 610 & 28 & 0.68 \\
\hline $4-29$ & $5-6$ & 47 & 590 & 21 & 0.78 \\
\hline $5-6$ & $5-13$ & 46 & 1000 & 44 & 1.4 \\
\hline $5-13$ & $5-20$ & 36 & 820 & 51 & 1.4 \\
\hline $5-20$ & $5-27$ & 29 & 820 & 43 & 1.3 \\
\hline $5-27$ & $6-3$ & 39 & 780 & 55 & 2.0 \\
\hline $6-3$ & $6-10$ & 29 & 630 & 63 & 1.7 \\
\hline 6.10 & $6-17$ & 30 & 500 & 84 & 1.8 \\
\hline $6-17$ & $6-24$ & 25 & 960 & 56 & 1.6 \\
\hline $6-24$ & $7-1$ & 29 & 790 & 34 & 1.4 \\
\hline $7=1$ & $7-8$ & 28 & 710 & 31 & 1.3 \\
\hline $7-8$ & $7 \cdot 15$ & 16 & 210 & 23 & 1.4 \\
\hline $7-15$ & $7-22$ & 3.8 & 44 & 18 & 1.3 \\
\hline $7-22$ & $7-29$ & 3.4 & 39 & 11 & 1.1 \\
\hline $7=29$ & $8-5$ & 2.8 & 32 & 9.3 & 0.93 \\
\hline $8-5$ & $8-12$ & 2.2 & 26 & 7.4 & 0.74 \\
\hline $8-12$ & $8-19$ & 2.0 & -23 & 6.6 & 0.66 \\
\hline $8=19$ & $8-26$ & 1.7 & 23 & 5.8 & 0.55 \\
\hline $8-26$ & 9.2 & 1.4 & $=27$ & 5.0 & 0.60 \\
\hline $9-2$ & $9-9$ & 7.7 & 590 & 4.5 & 0.65 \\
\hline $9-9$ & $9-16$ & 7.9 & 610 & 5.5 & 0.48 \\
\hline $9-16$ & $9-23$ & 11 & 590 & 4.5 & 0.69 \\
\hline $9-23$ & $9-30$ & 11 & 580 & 4.7 & 0.51 \\
\hline $9-30$ & $10-7$ & 14 & 432 & 4.5 & 0.47 \\
\hline $10-7$ & $10-14$ & 11 & 470 & 7.8 & 0.63 \\
\hline
\end{tabular}

No entry indicates no analysis made. 


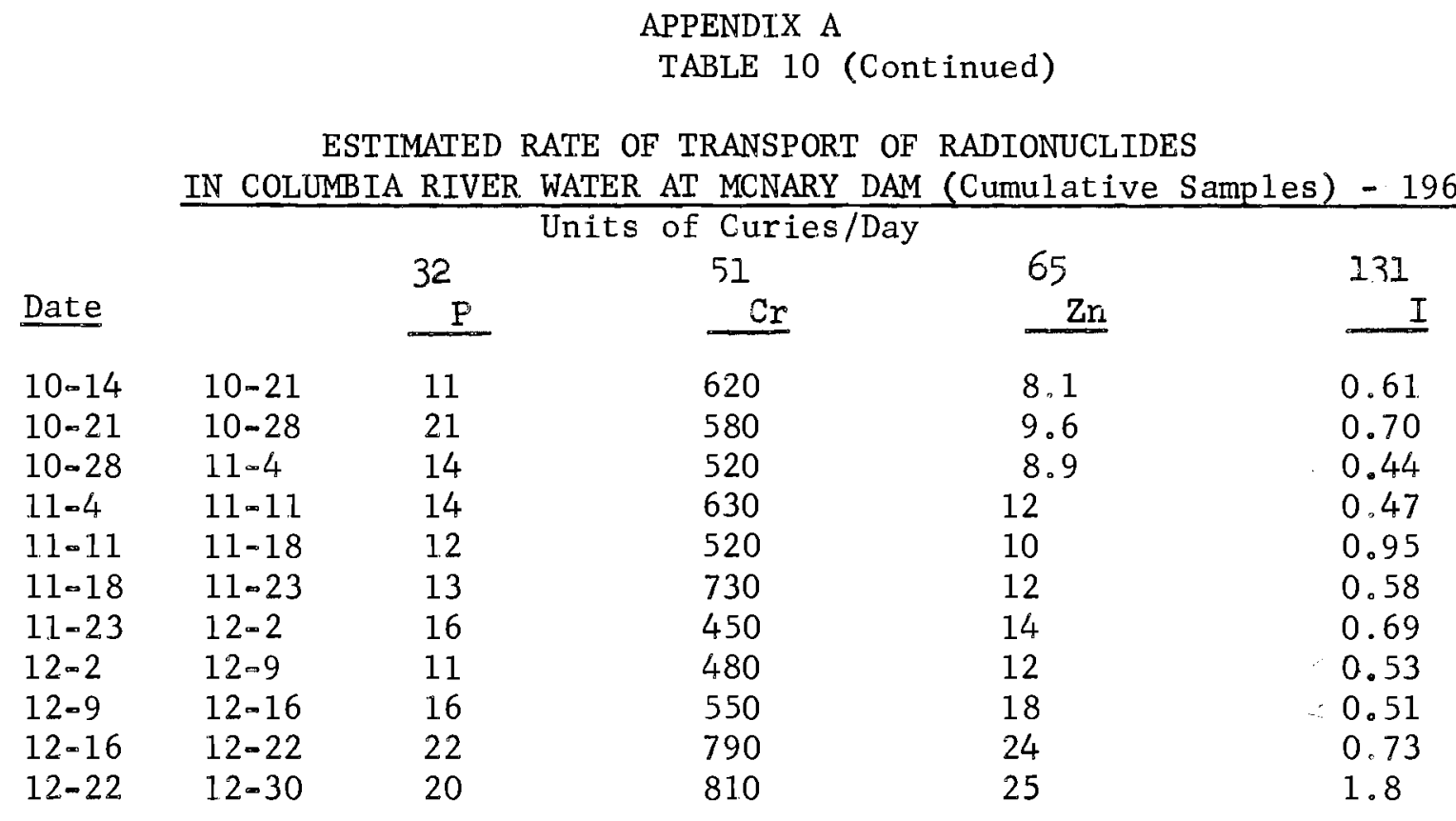

No entry indicates no analys is made. 
APPENDIX A

TABLE 11

\section{ESTIMATED RATE OF TRANSPORT OF RADIONUCLIDES IN COLIMBIA RIVER AT BONNEVILLE DAM (Cumulative Samp1es) - 1966}

\begin{tabular}{|c|c|c|c|c|c|}
\hline \multicolumn{2}{|l|}{ Date } & $\begin{array}{r}32 \\
P\end{array}$ & $51 \mathrm{Cr}$ & ${ }^{65} \mathrm{Zn}$ & ${ }_{I}^{131}$ \\
\hline \multicolumn{2}{|c|}{$12-28-65 / 1-4-66$} & 10 & 570 & 8.7 & 0.67 \\
\hline $1-4$ & $1-11$ & 10 & 600 & 17 & 0.51 \\
\hline $1=11$ & $1-25$ & 9.5 & 480 & 8.4 & 0.43 \\
\hline $1=25$ & $2-8$ & 12 & 600 & 10 & 1.2 \\
\hline $2-8$ & $2-22$ & 6.9 & 560 & 18 & 2.4 \\
\hline $2-22$ & $3-8$ & 13 & 480 & 8.4 & 7.8 \\
\hline $3=8$ & $3-22$ & 11 & 520 & 22 & 0.76 \\
\hline $3-22$ & $4-5$ & 16 & 620 & 25 & $<0.72$ \\
\hline $4-5$ & $4-19$ & 17 & 600 & 24 & 0.76 \\
\hline $4-19$ & $5-3$ & 13 & 500 & 20 & $<0.74$ \\
\hline $5-3$ & $5-17$ & 26 & 890 & 81 & $<1.3$ \\
\hline $5-17$ & $5-31$ & 25 & 750 & 70 & $<1.3$ \\
\hline $5-31$ & $6=14$ & 25 & 720 & 150 & $<1.8$ \\
\hline $6-14$ & $6-28$ & 20 & 1000 & 67 & $<1.6$ \\
\hline $6-28$ & $7-12$ & 19 & 640 & 51 & $<1.4$ \\
\hline $7=12$ & $7-19$ & & 200 & 47 & $<1.4$ \\
\hline 7.19 & $7-26$ & $<3.7$ & $<43$ & 21 & $<1.2$ \\
\hline $7-26$ & $8-2$ & $<3.2$ & $<38$ & $<11$ & $<1.1$ \\
\hline $8-2$ & $8-16$ & $<2.3$ & $<27$ & 13 & $<0.77$ \\
\hline $8=16$ & $8-23$ & $<1.9$ & $<22$ & 7.4 & $<0.62$ \\
\hline $8-23$ & $8-30$ & $<1.6$ & $<19$ & 8.0 & \\
\hline $8-30$ & $9-6$ & $<1.5$ & $<17$ & $<5.0$ & $<0.50$ \\
\hline $9-6$ & 9.13 & 1.6 & 220 & $<4.9$ & 0.67 \\
\hline $9-13$ & $9-20$ & 2.6 & 410 & $<4.7$ & 1.2 \\
\hline $9-20$ & $9-27$ & 3.4 & 410 & $<4.9$ & $<0.49$ \\
\hline $9-27$ & $10-4$ & 3.7 & 460 & $<5.0$ & 0.50 \\
\hline $10-4$ & $10-18$ & 5.0 & 310 & $<5.0$ & 0.50 \\
\hline $10-18$ & $10-25$ & 4.2 & 350 & $<4.7$ & $<0.47$ \\
\hline $10-25$ & $11-1$ & 5.9 & 450 & $<4.5$ & $<0.45$ \\
\hline $11-1$ & $11=8$ & 4.4 & 450 & $<4.9$ & $<0.49$ \\
\hline $11-8$ & $11-15$ & 4.8 & 390 & $<4.6$ & \\
\hline $11-1.5$ & $11-22$ & 9.7 & 430 & $<5.1$ & $<0.51$ \\
\hline $11-22$ & $11-29$ & 6.1 & 470 & $<4.9$ & 1.57 \\
\hline $11-29$ & $12-6$ & 5.9 & 320 & $<5.2$ & $<0.52$ \\
\hline $12-6$ & $12-13$ & 3.3 & 340 & $<5.9$ & $<0.59$ \\
\hline $12-13$ & $12-20$ & 6.5 & 370 & $<5.9$ & 0.94 \\
\hline $12-20$ & $12-27$ & 7.1 & 560 & 8.6 & $<0.62$ \\
\hline
\end{tabular}

No entry indicates no analysis made. 
BNWL-439 APP

\author{
APPENDIX B \\ RADIONUCLIDES IN DRINKING WATER
}




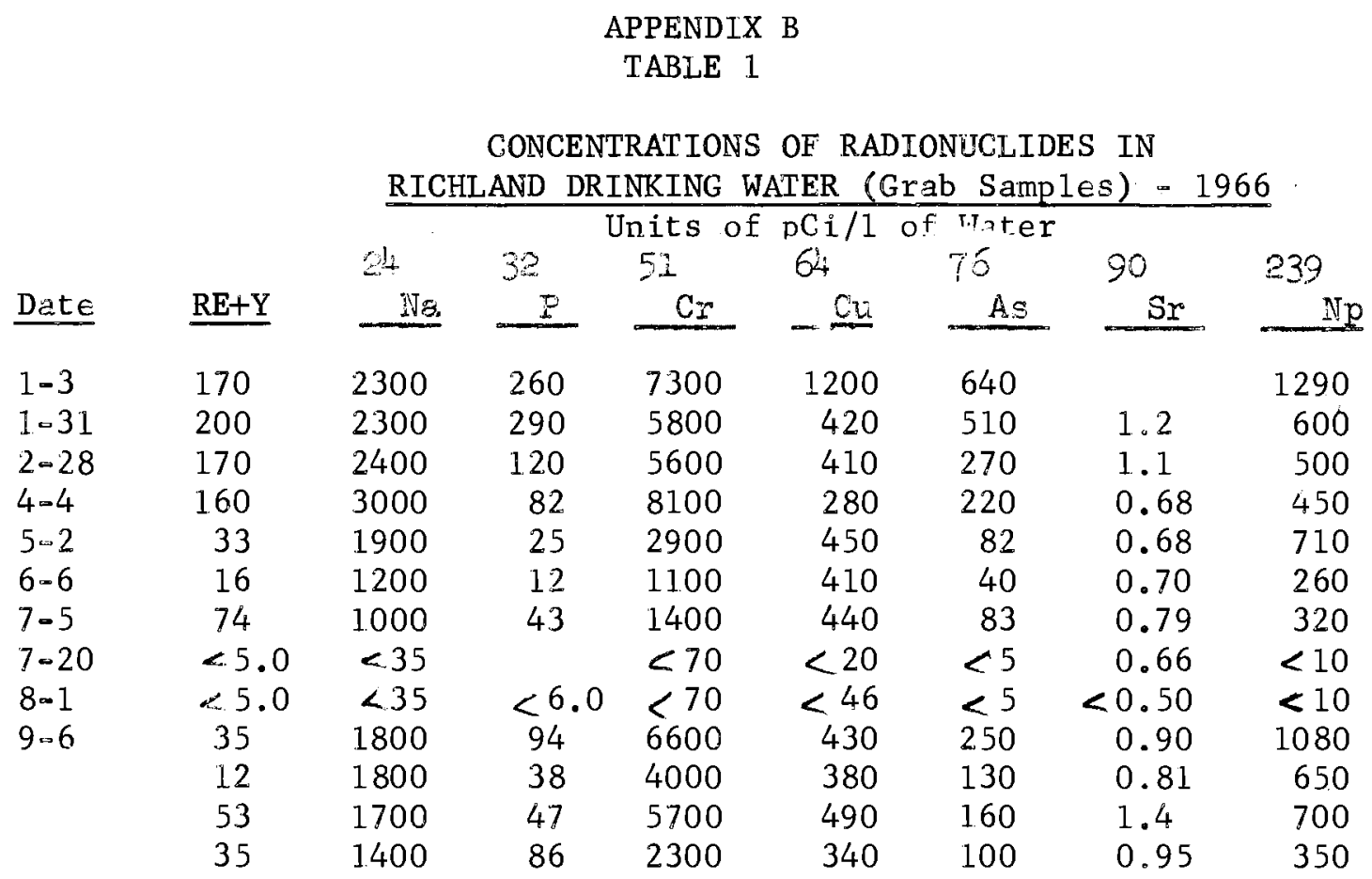

No entry indicates no analys is made. 


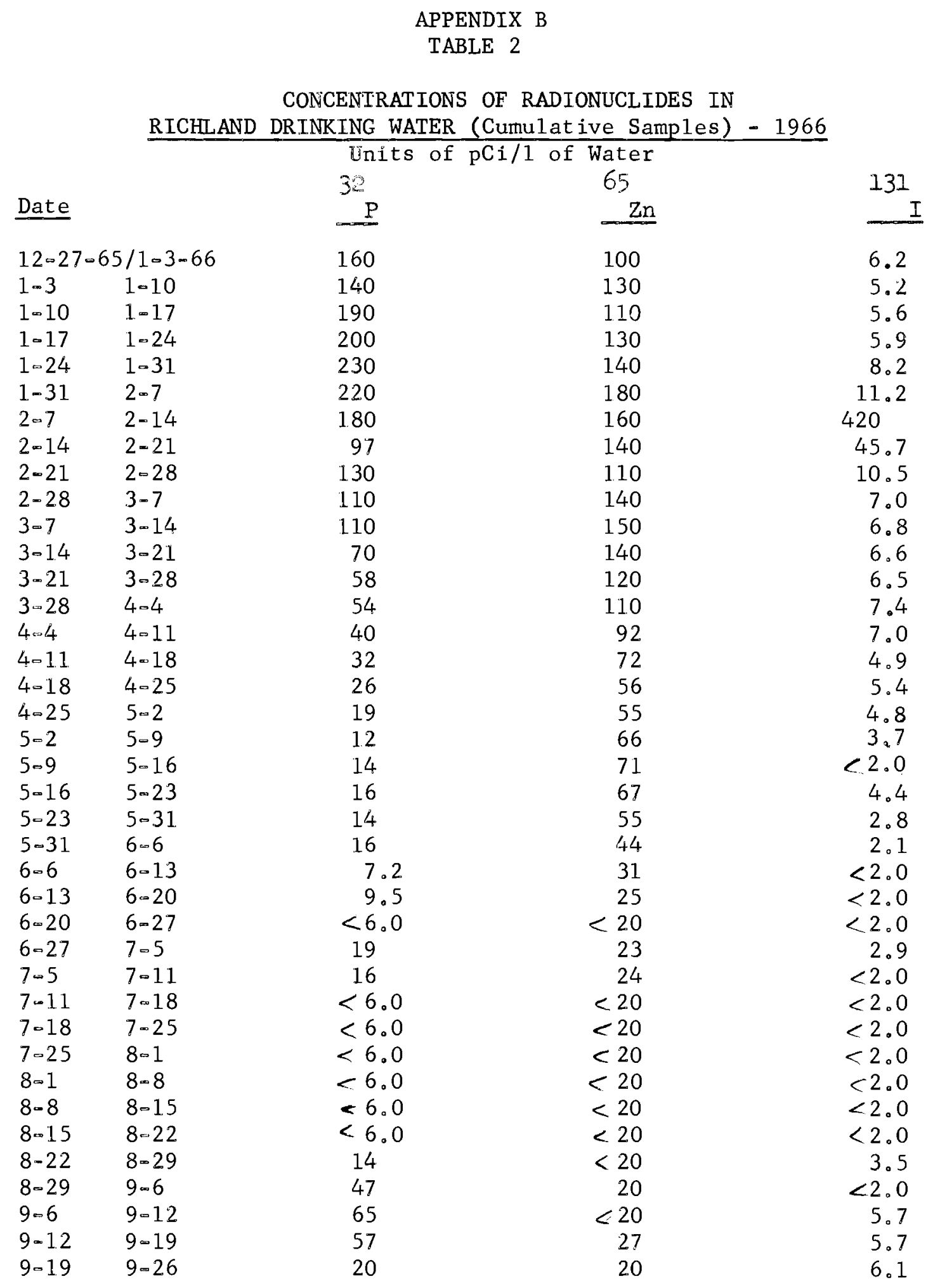

No entry indicates no analysis made. 
APPENDIX B

TABLE 2 (continued)

\section{CONCENTRATION OF RADIONUCLIDES IN RICHLAND}

DRINKING WATER (Cumulat ive Samples) - 1966

Date

9-26 10-3

$10-3 \quad 10-10$

$10=1.0 \quad 10-17$

$10-17 \quad 10-24$

$10-24 \quad 10-31$

$10-31 \quad 11-7$

$11-7 \quad 11-14$

$11-14 \quad 11=21$

$11-21 \quad 11-28$

$11-28 \quad 12-5$

$12=5 \quad 12-12$

$12-12 \quad 12-19$

$12-19 \quad 12-27$ Units of $\mathrm{pCi} / 1$ of Water

32

P

10

36

38

40

53

64

62

52

76

59

110

120

160

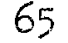

$\mathrm{Zn}$

20

21

32

31

27

49

50

51

56

64

95

95

130
131

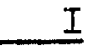

4.5

6.8

4.9

2.4

3.6

5.0

3.9

4.9

6.5

3.2

7.2

6.3

12.5

No entry indicates no analysis made. 


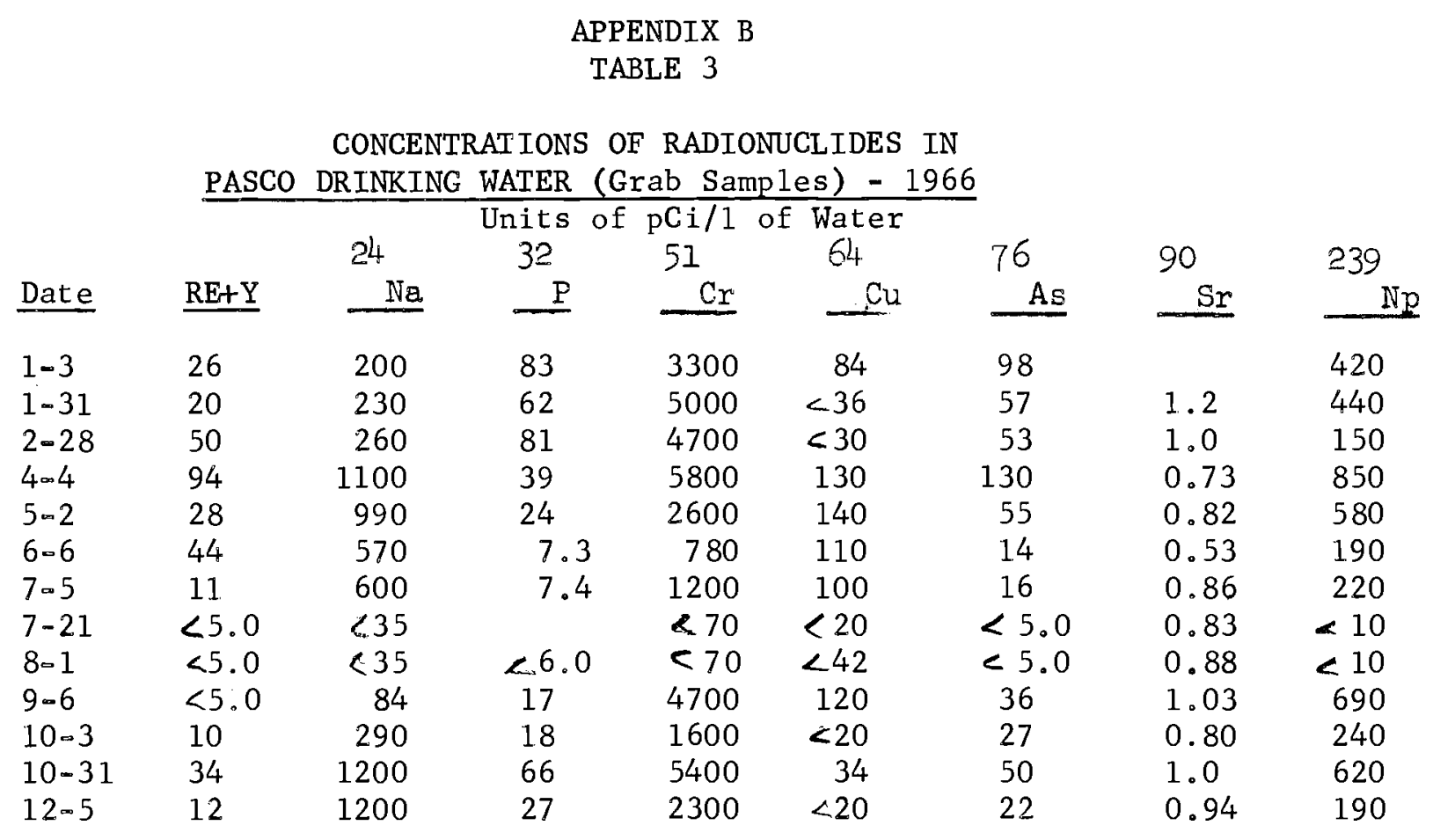

No entry indicates no analysis made. 
APPENDIX B

TABLE 4

CONCENTRATIONS OF RADIONUCLIDES IN

PASCO DRINKING WATER (Cumulative Samples) - 1966

\begin{tabular}{|c|c|c|c|c|}
\hline Date & & $\begin{array}{l}32 \\
? \\
\end{array}$ & $\begin{array}{l}65 \\
\mathrm{Zn} \\
\end{array}$ & $\begin{array}{r}132 \\
I \\
\end{array}$ \\
\hline $1-3$ & & 41 & 60 & 5.6 \\
\hline $1-10$ & & 180 & 69 & 5.8 \\
\hline $1-10-$ & $1-17$ & 45 & 75 & 4.1 \\
\hline $1-17$ & $1-24$ & 46 & 89 & 5.5 \\
\hline $1-24$ & $1-31$ & 38 & 78 & 8.9 \\
\hline $1-31$ & $2-7$ & 53 & 84 & 8.6 \\
\hline $2-7$ & $2-14$ & 45 & 110 & 50.8 \\
\hline $2-14$ & $2-21$ & 40 & 87 & 170 \\
\hline $2-21$ & $2-28$ & 18 & 160 & 12.1 \\
\hline $2-28$ & $3-7$ & 37 & 120 & 6.8 \\
\hline $3-7$ & $3-14$ & 34 & 110 & 5.3 \\
\hline $3=14$ & $3-21$ & 38 & 120 & 5.8 \\
\hline $3-21$ & $3-28$ & 39 & 94 & 4.6 \\
\hline $3=28$ & $4-4$ & 38 & 100 & 4.9 \\
\hline $4-4$ & $4-11$ & 47 & 98 & 6.1 \\
\hline $4-11$ & $4-18$ & 23 & 70 & 4.6 \\
\hline $4-18$ & $4=25$ & 21 & 37 & 3.7 \\
\hline $4-25$ & $5-2$ & 14 & 57 & 5.7 \\
\hline $5-2$ & 5.9 & 9.2 & 49 & 3.3 \\
\hline 5.9 & $5-16$ & 7.0 & 50 & 2.8 \\
\hline $5-16$ & $5-23$ & 6.1 & 52 & 3.9 \\
\hline $5-23$ & $5-31$ & $<6.0$ & 52 & 2.7 \\
\hline $5-31$ & 6.6 & 7.3 & 42 & $<2.0$ \\
\hline $6-6$ & $6-13$ & $<6.0$ & 30 & $<2.0$ \\
\hline $6-13$ & $6-20$ & 6.0 & 22 & 2.0 \\
\hline $6-20$ & $6-27$ & $<6.0$ & $<20$ & $<2.0$ \\
\hline $6-27$ & $7-5$ & 7.5 & $<20$ & 2.1 \\
\hline $7-5$ & $7-11$ & $<6.0$ & $<20$ & $<2.0$ \\
\hline $7-11$ & $7-18$ & $<6.0$ & $<20$ & $<2.0$ \\
\hline $7-18$ & $7-25$ & $<6.0$ & $<20$ & $<2.0$ \\
\hline $7-25$ & $8-1$ & $<6.0$ & $<20$ & $<2.0$ \\
\hline $8-1$ & $8-8$ & $<6.0$ & $<20$ & $<2.0$ \\
\hline $8-8$ & $8-15$ & $<6.0$ & $<20$ & $<2.0$ \\
\hline $8-15$ & $8 \cdot 22$ & $\angle 6.0$ & $<20$ & $<2.0$ \\
\hline $8-22$ & $8-29$ & 6.1 & $<20$ & 2.0 \\
\hline $8-29$ & 9.6 & 7.5 & $<20$ & 5.5 \\
\hline $9-6$ & $9-12$ & 6.1 & $<20$ & 6.0 \\
\hline $9-12$ & $9-19$ & 7.8 & 22 & 5.3 \\
\hline $9-19$ & $9-26$ & 13 & $<20$ & 5.9 \\
\hline
\end{tabular}

No entry indicates no analys is made. 


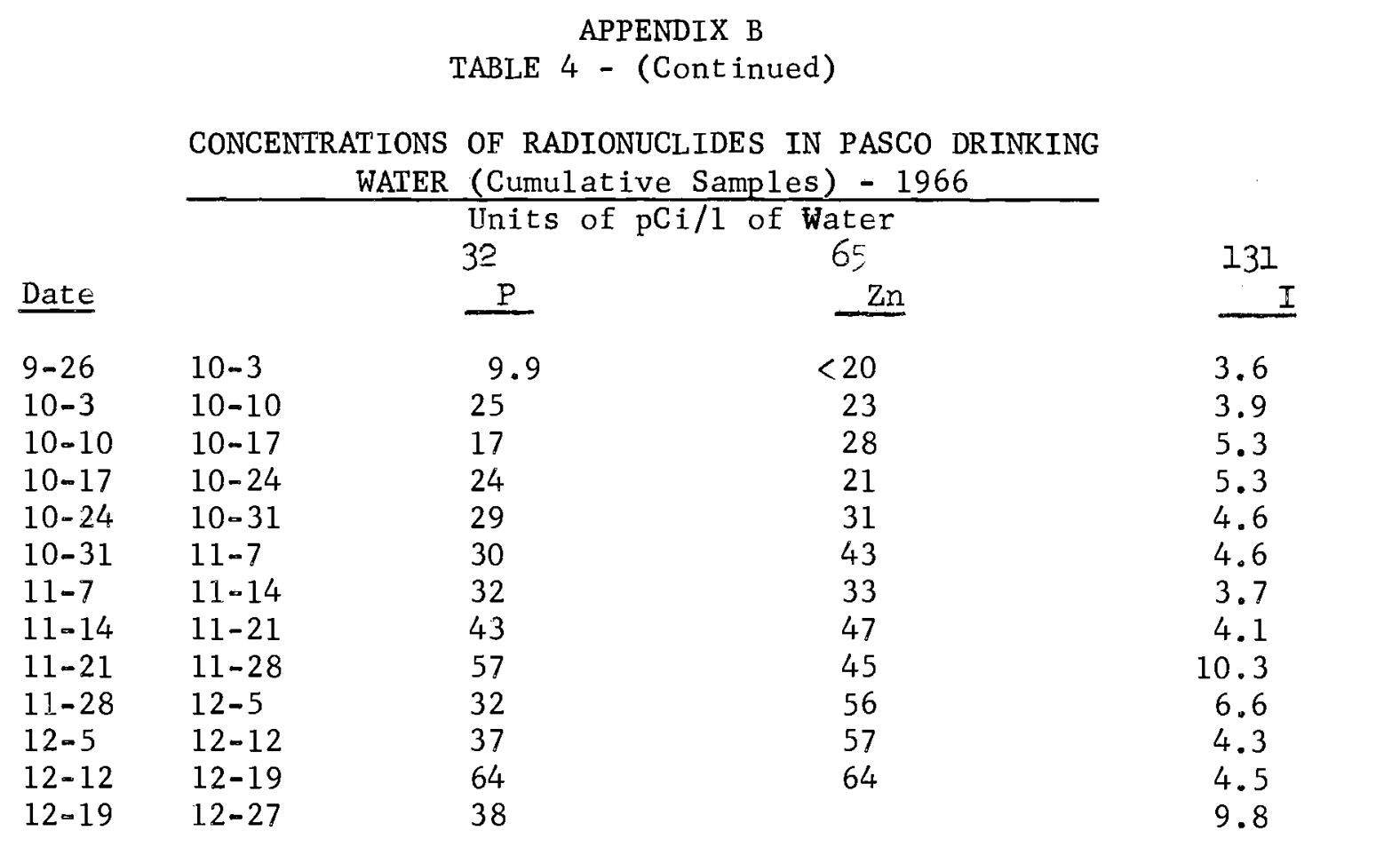

No entry indicates no analysis made. 
APPENDIX B

TABLE 5

CONCENTRATIONS OF RADIONUCLIDES IN KENNEWICK DRINKING WATER (Grab Samples) - 1966

\begin{tabular}{|c|c|c|c|c|c|c|}
\hline Date & $\begin{array}{l}2^{2} \\
\quad N \varepsilon \\
\end{array}$ & $\begin{array}{r}32 \\
P \\
\end{array}$ & $\begin{array}{l}5 . \\
u_{r}\end{array}$ & $\begin{array}{r}64 \\
\quad 0 \\
\end{array}$ & $\begin{array}{l}76 \\
\text { As } \\
\end{array}$ & $\begin{array}{l}239 \\
\quad \mathrm{~Np} \\
\end{array}$ \\
\hline $1-17$ & 58 & 15.9 & 2100 & $<38$ & 17 & 55 \\
\hline $2-14$ & 44 & 13.7 & 2200 & $\therefore 20$ & 12 & 250 \\
\hline $3-14$ & 50 & 7.4 & 1500 & $<20$ & 10 & 25 \\
\hline $4-18$ & 39 & 8.5 & 1400 & $<20$ & 7.3 & 23 \\
\hline $5=16$ & 94 & 12.4 & 780 & 55 & 14.8 & 52 \\
\hline $6-20$ & 66 & $<6.0$ & 620 & 46 & 9.0 & 29 \\
\hline $7-18$ & $<35$ & $<6.0$ & 75 & $<20$ & $<5.0$ & $<10$ \\
\hline $8-15$ & $<35$ & $<6.0$ & $<70$ & -38 & $<6.0$ & $<10$ \\
\hline $9-19$ & 78 & 8.1 & 1800 & 48 & 9.9 & 47 \\
\hline $10-17$ & 47 & 9.9 & 1600 & $<20$ & 10 & 40 \\
\hline $11-14$ & 120 & 19.2 & 2100 & 65 & 18 & 78 \\
\hline $12-19$ & 110 & 19.1 & 1900 & 81 & 24 & 66 \\
\hline
\end{tabular}

No entry indicates no analysis made. 


\section{APPENDIX B}

TABLE 6

BETA ACTIVITY IN RICHLAND DRINKING WATER - 1966

\section{Units of $\mathrm{c} / \mathrm{m} / \mathrm{m} 1$}

\begin{tabular}{|c|c|c|c|c|c|}
\hline Date & $\mathrm{c} / \mathrm{m} / \mathrm{m} 1$ & Date & $\mathrm{c} / \mathrm{m} / \mathrm{ml}$ & Date & $\mathrm{c} / \mathrm{m} / \mathrm{ml}$ \\
\hline $1-3$ & 8.6 & $6-20$ & 2.40 & $9-20$ & 3.7 \\
\hline $1-4$ & 8.8 & $6-21$ & 2.44 & $9-28$ & 4.6 \\
\hline $1-5$ & 7.0 & $6-22$ & 1.84 & $9-29$ & 6.5 \\
\hline $1-6$ & 6.4 & $6-23$ & 2.44 & $10-3$ & 4.6 \\
\hline $1-7$ & 5.7 & $6-24$ & 0.90 & $10-4$ & 4.6 \\
\hline $1=10$ & 4.9 & $6-27$ & 3.20 & $10-11$ & 4.76 \\
\hline $1-11$ & 7.0 & $6-28$ & 2.60 & $10-18$ & 8.82 \\
\hline $1-12$ & 4.0 & $6-29$ & 1.50 & $10-25$ & 4.04 \\
\hline $1-13$ & 2.0 & $6-30$ & 1.74 & $10-31$ & 1.70 \\
\hline $1-19$ & 9.60 & $7-1$ & 2.30 & $11-2$ & 4.40 \\
\hline $1-26$ & 8.20 & 7.5 & 2.50 & $11-14$ & 5.40 \\
\hline $1-31$ & 6.82 & $7-6$ & 1.00 & $11-22$ & 4.90 \\
\hline $2-9$ & 7.20 & $7-7$ & 1.82 & $11-28$ & 2.46 \\
\hline $2-16$ & 2.64 & $7-8$ & 1.20 & $12-5$ & 2.30 \\
\hline $2-23$ & 6.84 & $7-11$ & 0.20 & $12-6$ & 6.50 \\
\hline $2-28$ & 6.00 & $7-12$ & 0.20 & $12-7$ & 6.08 \\
\hline $3-9$ & 7.96 & $7-13$ & 0.20 & $12-8$ & 5.90 \\
\hline $3-16$ & 9.92 & $7-14$ & 0.20 & $12-9$ & 8.00 \\
\hline $3-23$ & 7.64 & $7-15$ & 0.20 & $12-13$ & 13.6 \\
\hline $3-30$ & 7.92 & $7-18$ & 0.02 & $12-19$ & 5.06 \\
\hline $4-4$ & 7.90 & $7-20$ & 0.02 & $12-20$ & 9.64 \\
\hline $4-13$ & 9.24 & $7-25$ & 0.02 & $12-21$ & 10.2 \\
\hline $4-2.0$ & 6.40 & $8-1$ & 0.02 & $12-22$ & 10.4 \\
\hline $4-27$ & 3.72 & $8-5$ & 0.02 & $12-27$ & 8.4 \\
\hline $5-2$ & 4.20 & $8-10$ & 0.02 & & \\
\hline $5-11$ & 5.96 & $8-15$ & 0.005 & & \\
\hline $5-18$ & 5.76 & $8-19$ & 0.005 & & \\
\hline $5-25$ & 3.14 & $8-22$ & 0.005 & & \\
\hline $5-31$ & 1.22 & $8-23$ & 0.02 & & \\
\hline $6-1$ & 3.16 & $8-24$ & 0.02 & & \\
\hline $6-2$ & 3.16 & $8-25$ & 0.53 & & \\
\hline $6-3$ & 3.24 & $8=26$ & 0.93 & & \\
\hline $6-6$ & 2.88 & $8-27$ & 4.2 & & \\
\hline $6-8$ & 1.40 & $8-28$ & 4.8 & & \\
\hline $6-9$ & 0.90 & $8-29$ & 3.2 & & \\
\hline $6-10$ & 1.30 & $8-30$ & 5.2 & & \\
\hline $6-13$ & 2.12 & $8-31$ & 3.2 & & \\
\hline $6-15$ & 2.08 & $9-6$ & 6.0 & & \\
\hline $6-16$ & 2.34 & $9-8$ & 4,6 & & \\
\hline $6-17$ & 0.72 & $9-13$ & 5.9 & & \\
\hline
\end{tabular}




\section{APPENDIX B}

TABLE 7

BETA ACTIVITY IN PASCO DRINKING WATER - 1966

$$
\text { Units of } \mathrm{c} / \mathrm{m} / \mathrm{ml}
$$

\begin{tabular}{|c|c|c|c|c|c|}
\hline Date & $\mathrm{c} / \mathrm{m} / \mathrm{m} 1$ & Date & $\mathrm{c} / \mathrm{m} / \mathrm{m} 1$ & Date & $\mathrm{c} / \mathrm{m} / \mathrm{m} 1$ \\
\hline $1-3$ & 1.34 & $3-2$ & 1.82 & $4-28$ & 2.46 \\
\hline $1-4$ & 2.30 & $3-3$ & 1.68 & $4-29$ & 2.40 \\
\hline $1-5$ & 1.64 & $3-7$ & 1.17 & $5-2$ & 2.94 \\
\hline $1-6$ & 1.86 & $3-8$ & 2.00 & $5-3$ & 2.76 \\
\hline $1-7$ & 1.42 & $3-9$ & 2.28 & $5-4$ & 2.06 \\
\hline $1-10$ & 1.64 & $3-10$ & 1.85 & $5-5$ & 2.54 \\
\hline $1-11$ & 1.02 & $3-11$ & 2.56 & $5-6$ & 2.38 \\
\hline $1=12$ & 1.04 & $3 \cdot-14$ & 1.52 & $5-9$ & 3.06 \\
\hline $1-13$ & 2.0 & $3-15$ & 1.70 & $5-10$ & 2.92 \\
\hline $1-1.7$ & 1.16 & $3-16$ & 1.80 & $5-11$ & 3.78 \\
\hline $1-18$ & 1.60 & $3-17$ & 2.12 & $5-12$ & 2.46 \\
\hline $1-19$ & 1.02 & $3-18$ & 3.12 & $5-13$ & 2.92 \\
\hline $1-20$ & 1.60 & $3-21$ & 1.32 & $5-16$ & 1.74 \\
\hline $1-21$ & 1.30 & $3-22$ & 1.02 & $5-17$ & 0.77 \\
\hline $1-24$ & 1.18 & $3-23$ & 2.36 & $5-18$ & 2.50 \\
\hline $1-2,5$ & 0.52 & $3-24$ & 1.72 & $5-19$ & 3.50 \\
\hline $1-26$ & 0.52 & $3-25$ & 2.22 & $5-20$ & 2.54 \\
\hline $1-27$ & 0.48 & $3-28$ & 3.54 & $5-23$ & 3.10 \\
\hline $1-28$ & 1.14 & $3-29$ & 2.96 & $5-24$ & 2.14 \\
\hline $1-31$ & 1.52 & $3-3-$ & 3.54 & $5-25$ & 2.44 \\
\hline $2-1$ & 1.07 & $3-31$ & 3.00 & $5-26$ & 1.70 \\
\hline $2-2$ & 1.26 & $4-1$ & 2.24 & $5-27$ & 1.48 \\
\hline $2-3$ & 1.84 & $4-4$ & 3.90 & $5-31$ & 2.20 \\
\hline 2.4 & 2.00 & $4-5$ & 5.14 & $6-1$ & 1.76 \\
\hline $2-7$ & 1.62 & $4-6$ & 5.80 & $6-2$ & 1.88 \\
\hline $2-8$ & 0.96 & $4-7$ & 4.72 & $6-3$ & 1.38 \\
\hline $2-9$ & 7.20 & $4-8$ & 4.64 & $6-6$ & 1.31 \\
\hline $2-10$ & 2.50 & $4-11$ & 4.30 & $6-7$ & 1.00 \\
\hline $2-14$ & 2.14 & $4-12$ & 3.16 & $6-8$ & 0.83 \\
\hline $2-15$ & 1.44 & $4-13$ & 2.74 & $6-9$ & 0.40 \\
\hline $2-16$ & 1.46 & $4-14$ & 2.70 & $6-10$ & 0.88 \\
\hline $2-17$ & 0.82 & $4-15$ & 2.15 & $6-13$ & 0.98 \\
\hline $2-18$ & 0.60 & $4-18$ & 1.70 & $6-14$ & 1.56 \\
\hline $2-21$ & 0.76 & $4-19$ & 2.48 & $6-1.5$ & 1.00 \\
\hline $2-22$ & 0.98 & 4.20 & 2.82 & $6-16$ & 1.47 \\
\hline $2-23$ & 2.80 & $4-21$ & 2.66 & $6-17$ & 1.33 \\
\hline $2-2.4$ & 1.77 & 4.22 & 1.98 & $6-20$ & 1.33 \\
\hline $2-25$ & 1. 32 & $4-25$ & 2.90 & $6-21$ & 1.51 \\
\hline $2-28$ & 1.37 & $4-26$ & 3.74 & $6-22$ & 0.90 \\
\hline $3-1$ & 1.59 & $4-27$ & 2.80 & $6-23$ & 1.04 \\
\hline
\end{tabular}


APPENDIX B

TABLE 7 (Cont inued)

BETA ACTIVITY IN PASCO DRINKING WATER - 1966

Units of $\mathrm{c} / \mathrm{m} / \mathrm{ml}$

\begin{tabular}{|c|c|c|c|c|c|}
\hline Date & $\mathrm{c} / \mathrm{m} / \mathrm{m} 1$ & Date & $\mathrm{c} / \mathrm{m} / \mathrm{ml}$ & Date & $\mathrm{c} / \mathrm{m} / \mathrm{ml}$ \\
\hline $6-24$ & 0.46 & $9-15$ & 1.5 & $11-11$ & 1.3 \\
\hline $6-27$ & 1.66 & $9-16$ & 2.7 & $11-14$ & 1.5 \\
\hline $6-28$ & 1.48 & $9-19$ & 2.5 & $11-15$ & 1.4 \\
\hline $6-29$ & 1.02 & $9-20$ & 2.2 & $11-16$ & 2.0 \\
\hline $6-30$ & 0.78 & $9-21$ & 2.6 & $11-17$ & 1.9 \\
\hline $7-1$ & 1.18 & $9-22$ & 2.0 & $11-18$ & 1.7 \\
\hline $7-5$ & 1.12 & $9-23$ & 2.6 & $11-21$ & 1.3 \\
\hline $7-6$ & 1.32 & $9-26$ & 2.4 & $11-22$ & 1.0 \\
\hline $7-7$ & 1.26 & $9-28$ & 2.8 & $11-23$ & 1.1 \\
\hline $7-8$ & 1.12 & $9-29$ & 2.3 & $11-28$ & 1.82 \\
\hline $7-11$ & -0.2 & $9-20$ & 2.1 & $11-29$ & 1.10 \\
\hline $7-12$ & 0.2 & $10-3$ & 1.0 & $11-30$ & 1.33 \\
\hline $7-13$ & 0.2 & $10-4$ & 1.6 & $12-1$ & 1.50 \\
\hline $7-14$ & 0.2 & $10-5$ & 1.9 & $12-2$ & 0.97 \\
\hline $7-15$ & .0 .2 & $10-6$ & 2.1 & $12-5$ & 0.76 \\
\hline $7-18$ & 0.02 & 10.7 & 2.0 & $12-6$ & 0.45 \\
\hline $7-21$ & 0.02 & $10-10$ & 1.7 & $12-7$ & 1.82 \\
\hline $7-25$ & 0.02 & $10=11$ & 2.5 & $12-8$ & 2.60 \\
\hline $8-1$ & 0.02 & $10-12$ & 2.4 & $12-9$ & 3.10 \\
\hline $8-5$ & 0.02 & $10-13$ & 2.7 & $12-12$ & 1.26 \\
\hline $8-12$ & 0.004 & $10-14$ & 1.8 & $12-13$ & 0.32 \\
\hline $8-15$ & 0.004 & $10-17$ & 2.0 & $12-14$ & 1.95 \\
\hline $8-1.9$ & 0.004 & $10-18$ & 2.6 & $12-15$ & 1.86 \\
\hline $8-22$ & 0.005 & $10-19$ & 3.0 & $12-16$ & 0.38 \\
\hline $8 \cdot 23$ & $<0.001$ & $10-20$ & 1.6 & $12-19$ & 1.75 \\
\hline $8-24$ & 0.01 & $10-21$ & 1.9 & $12-2-$ & 1.14 \\
\hline $8-25$ & 1.14 & $10-24$ & 1.1 & $12-21$ & 1.21 \\
\hline $8-26$ & 0.62 & $10=25$ & 1.43 & $12-22$ & 1.60 \\
\hline $8-29$ & 2.5 & $10-26$ & 1.90 & $12-27$ & 1.60 \\
\hline $8-20$ & 3.22 & $10-27$ & 1.68 & $12-28$ & 1.30 \\
\hline $8-31$ & 1.14 & $10-28$ & 1.37 & $12-29$ & 2.16 \\
\hline $9-1$ & 2.14 & $10-31$ & 1.66 & $12-30$ & 1.94 \\
\hline $9-2$ & 2.7 & $11-1$ & 1.61 & & \\
\hline $9-6$ & 2.0 & $11-2$ & 1.14 & & \\
\hline $9-7$ & 3.3 & $11-3$ & 1.12 & & \\
\hline $9-8$ & 2.8 & $11-4$ & 0.94 & & \\
\hline 9.9 & 2.0 & $11-7$ & 1.31 & & \\
\hline $9-12$ & 1.3 & $11-8$ & 1.5 & & \\
\hline $9-13$ & 1.1 & $11-9$ & 0.97 & & \\
\hline $9-14$ & 1.8 & $11-10$ & 1.9 & & \\
\hline
\end{tabular}


APPENDIX B

TABLE 8

BETA ACTIVITY IN KENNEWICK DRINKING WATER - 1966

Units of $\mathrm{c} / \mathrm{m} / \mathrm{m} 1$

\begin{tabular}{|c|c|c|c|c|c|}
\hline Date & $\mathrm{c} / \mathrm{m} / \mathrm{ml}$ & Date & $\mathrm{c} / \mathrm{m} / \mathrm{m} 1$ & Date & $\mathrm{c} / \mathrm{m} / \mathrm{m} 1$ \\
\hline $1-3$ & 0.29 & $3-3$ & 0.28 & $4-28$ & 0.63 \\
\hline $1-4$ & 0.33 & $3-4$ & $<0.10$ & $4-29$ & 0.55 \\
\hline 1.5 & 0.31 & $3-7$ & 0.35 & $5-2$ & 0.48 \\
\hline $1-6$ & 0.27 & $3-8$ & 0.37 & $5-3$ & 0.59 \\
\hline $1-7$ & 0.28 & $3-9$ & 0.35 & $5-4$ & 0.47 \\
\hline 1.10 & 0.29 & $3-10$ & 0.35 & $5-5$ & 0.71 \\
\hline $1-11$ & 0.25 & $3-11$ & 0.23 & $5=6$ & 0.51 \\
\hline $1-12$ & 0.29 & $3-14$ & 0.24 & $5=9$ & 0.60 \\
\hline $1-13$ & 0.35 & $3-15$ & 0.29 & $5-10$ & 0.58 \\
\hline $1-17$ & 0.34 & $3=16$ & 0.24 & $5-11$ & 0.29 \\
\hline $1-19$ & 0.73 & $3=17$ & 0.26 & $5-12$ & 0.75 \\
\hline $1-20$ & 0.61 & $3-18$ & 0.33 & $5-13$ & 0.43 \\
\hline $1-21$ & 1.25 & $3=21$ & 0.28 & $5-16$ & 0.36 \\
\hline $1-24$ & 0.36 & $3-22$ & 0.32 & $5-17$ & 0.37 \\
\hline $1=25$ & 0.72 & $3-23$ & 0.29 & $5-18$ & 0.44 \\
\hline $1-26$ & 1.15 & $3=24$ & 0.26 & $5-19$ & 0.38 \\
\hline $1=27$ & 0.37 & $3-25$ & 0.22 & $5-20$ & 0.38 \\
\hline $1-28$ & 0.62 & $3-28$ & 0.20 & $5-23$ & 0.46 \\
\hline $1-31$ & 0.38 & $3-29$ & 0.21 & $5 \cdot 24$ & 0.42 \\
\hline $2-1$ & 0.46 & 3.30 & 0.26 & $5-25$ & 0.32 \\
\hline $2-2$ & 0.40 & $3-31$ & 0.25 & $5-26$ & 0.36 \\
\hline $2-3$ & 0.38 & $4-1$ & 0.29 & $5=27$ & 0.38 \\
\hline $2-4$ & 0.45 & $4-4$ & 0.22 & $5-31$ & 0.27 \\
\hline $2-7$ & 0.50 & $4-5$ & 0.31 & $6-1$ & 0.34 \\
\hline 2008 & 0.56 & $4-6$ & 0.26 & $6-2$ & 0.18 \\
\hline $2-9$ & 0.50 & $4-7$ & 0.24 & $6-3$ & 0.15 \\
\hline $2-10$ & 0.39 & $4-8$ & 0.23 & $\begin{array}{l}0.3 \\
6-6\end{array}$ & $\begin{array}{lll}0 & 10 \\
0 & 24\end{array}$ \\
\hline $2=14$ & $\begin{array}{r}.02 \\
-.24\end{array}$ & $4-11$ & 0.27 & $6-7$ & $\begin{array}{l}0.24 \\
0.24\end{array}$ \\
\hline $2-15$ & 0.26 & $4=12$ & 0.49 & $6-8$ & 0.16 \\
\hline $2 \Leftrightarrow 16$ & 0.28 & $4-13$ & 0.62 & $6-9$ & 0.13 \\
\hline $2=17$ & 0.24 & $4-14$ & 0.70 & $6-10$ & 0.11 \\
\hline $2-18$ & 0.18 & $4-15$ & 0.28 & $6-13$ & 0.28 \\
\hline $2-21$ & 0.30 & $4-18$ & 0.23 & $6-14$ & 0.18 \\
\hline $2-22$ & 0.47 & $4=19$ & 0.29 & $6-15$ & 0.14 \\
\hline $2-23$ & 0.39 & $4=20$ & 0.29 & $6-16$ & 0.19 \\
\hline $2-24$ & 0.41 & $4-21$ & 0.23 & $6-17$ & 0.10 \\
\hline $2-25$ & 0.30 & $4-22$ & 0.62 & $6-20$ & 0.35 \\
\hline $2-28$ & 0.24 & $4-25$ & 0.43 & $6-21$ & 0.25 \\
\hline $3-1$ & 0.49 & $4-26$ & 0.39 & $6-22$ & 0.20 \\
\hline $3-2$ & 0.30 & $4-27$ & 0.56 & $6-23$ & 0.13 \\
\hline
\end{tabular}


APPENDIX B

TABLE 8 (Continued)

BETA ACTIVITY IN KENNEWICK DRINKING WATER - 1966 Units of $\mathrm{c} / \mathrm{m} / \mathrm{ml}$

\begin{tabular}{|c|c|c|c|c|c|}
\hline Date & $\mathrm{c} / \mathrm{m} / \mathrm{ml}$ & Date & $\mathrm{c} / \mathrm{m} / \mathrm{ml}$ & Date & $\mathrm{c} / \mathrm{m} / \mathrm{m} 1$ \\
\hline $6-24$ & 0.12 & $9-15$ & 0.21 & $11-11$ & 0.21 \\
\hline $6-27$ & 0.23 & $9-16$ & 0.18 & $11-14$ & 0.37 \\
\hline $6-28$ & 0.24 & $9-19$ & 0.30 & $11-15$ & 0.55 \\
\hline $6-29$ & 0.21 & $9-20$ & 0.16 & $11-16$ & 0.60 \\
\hline $6-30$ & 0.17 & $9-21$ & 0.23 & $11-17$ & 0.40 \\
\hline $7-1$ & 0.20 & $9-22$ & 0.36 & $11-18$ & 0.49 \\
\hline $7-5$ & 0.18 & $9-23$ & 0.37 & $11-21$ & 0.49 \\
\hline $7-6$ & 0.22 & $9-26$ & 0.36 & $11-22$ & 0.39 \\
\hline $7-7$ & 0.13 & $9-28$ & 0.34 & $11-23$ & 0.45 \\
\hline $7-8$ & $\therefore 0.05$ & $9-29$ & 0.49 & $11-28$ & 0.33 \\
\hline $7-11$ & 0.05 & $9-30$ & 0.41 & $11-29$ & 0.55 \\
\hline $7-12$ & 0.05 & $10-3$ & 0.31 & $11-30$ & 0.41 \\
\hline $7-13$ & 0.05 & $10-4$ & 0.40 & $12-1$ & 0.29 \\
\hline $7-14$ & 0.15 & $10-5$ & 0.19 & $12-2$ & 0.27 \\
\hline $7-15$ & 0.11 & $10-6$ & 0.14 & $12-5$ & 0.27 \\
\hline $7-18$ & $=0.05$ & $10-7$ & 0.30 & $12-6$ & 0.56 \\
\hline $7-21$ & 0.02 & $10-10$ & 0.42 & $12-7$ & 0.50 \\
\hline $7-25$ & 0.02 & $10-11$ & 0.37 & $12-8$ & 0.55 \\
\hline $8-1$ & 0.02 & $10-12$ & 0.41 & $12-9$ & 0.55 \\
\hline $8-5$ & 0.02 & $10-13$ & 0.39 & $12-12$ & 0.41 \\
\hline $8-12$ & 0.004 & $10-14$ & 0.39 & $12-13$ & 0.69 \\
\hline $8-15$ & 0.004 & $10-17$ & 0.26 & $12-14$ & 0.54 \\
\hline $8-19$ & 0.003 & $10-18$ & 0.60 & $12-15$ & 0.35 \\
\hline $8-22$ & 0.003 & $10-19$ & 0.36 & $12-16$ & 0.32 \\
\hline $8-23$ & 0.01 & $10-20$ & 0.21 & $12-19$ & 0.41 \\
\hline $8-24$ & 0.01 & $10-21$ & 0.26 & $12-20$ & 0.76 \\
\hline $8=25$ & 0.003 & $10-24$ & 0.30 & $12-21$ & 0.66 \\
\hline $8-26$ & 0.004 & $10-25$ & 0.46 & $12-22$ & 0.62 \\
\hline $8-29$ & 0.33 & $10-26$ & 0.40 & $12-27$ & 0.76 \\
\hline $8-30$ & 0.41 & $10-27$ & 0.36 & $1.2-28$ & 0.97 \\
\hline $8-31$ & 0.43 & $10-28$ & 0.37 & $12-29$ & 0.60 \\
\hline $9-1$ & 0.26 & $10-31$ & 0.37 & $12-30$ & 0.35 \\
\hline $9-2$ & 0.29 & $11-1$ & 0.33 & & \\
\hline $9-6$ & 0.42 & $11-2$ & 0.36 & & \\
\hline $9-7$ & 0.47 & $11-3$ & 0.29 & & \\
\hline $9-8$ & 0.41 & $11-4$ & 0.29 & & \\
\hline $9-9$ & 0.44 & $11-7$ & 0.39 & & \\
\hline $9-12$ & 0.16 & $11-8$ & 0.28 & & \\
\hline $9-13$ & 0.20 & $11-9$ & 0.31 & & \\
\hline $9-14$ & 0.21 & $11-10$ & 0.26 & & \\
\hline
\end{tabular}


BNWL-439 APP

APPENDIX C

RADIONUCLIDES IN FISH AND WATERFOWL 
APPENDIX C

TABLE 1

CONCENTRATIONS OF RADIONUCLIDES IN MUSCLE

OF WHITEF ISH TAKEN FROM THE COLUMBIA RIVER - 1966

Units of picocuries per gram

\begin{tabular}{|c|c|c|c|c|c|c|}
\hline Date & $32_{\mathrm{P}}$ & مC 60 & $40_{K}$ & $65 \mathrm{~min}$ & ${ }^{58} \mathrm{C}$ & $137 \mathrm{Cs}$ \\
\hline Limit & 1 & 0.2 & 1 & 0.2 & 0.2 & 0.1 \\
\hline
\end{tabular}

Priest Rapids

$\begin{array}{lrllcll}1-8 & 4 & 0.2 & 6 & 6.0 & - & - \\ 10-5 & 5 & & 4 & 0.3 & & 0.2 \\ 11-22 & 2 & & 5 & 1.3 & & 0.2 \\ 11-22 & 29 & & 4 & 0.4 & & 0.2 \\ 11-22 & 43 & 0.9 & 4 & 14 & 0.4 & 0.4 \\ 11-22 & 3 & & 4 & 0.5 & & 0.1 \\ 11-22 & 19 & 0.3 & 4 & 12 & 0.2 & 0.3 \\ 11-22 & 5 & 0.4 & 5 & 5.2 & - & 0.2 \\ 11-22 & 73 & 1.1 & 3 & 17 & 0.5 & 0.5 \\ 11-22 & 4 & 0.6 & 5 & 5.5 & 0.2 & 0.5 \\ 11-22 & 1.20 & 1.1 & 5 & 24 & 0.3 & 0.7 \\ 11-22 & 66 & 0.5 & 4 & 12 & 0.2 & 0.4 \\ 11-22 & 63 & 0.5 & 4 & 17 & 0.3 & 0.3 \\ 11-22 & 220 & 0.7 & 5 & 17 & 0.4 & 0.3 \\ 11-22 & 160 & 0.7 & 6 & 26 & 0.3 & 0.4 \\ 11-22 & - & & 4 & 0.7 & & 0.3\end{array}$

Hanford

$\begin{array}{lrlllll}1-7 & 44 & 4.2 & 5 & 32 & 1.3 & 0.8 \\ 1-7 & 1 & 0 & 2 & - & - & 0.4 \\ 1-7 & 100 & 2.7 & 5 & 41 & 2.0 & 0.6 \\ 1-7 & 53 & 2.9 & 5 & 48 & 1.8 & 0.6 \\ 1-7 & 51 & 2.3 & 4 & 31 & 1.1 & 0.7 \\ 1-7 & 69 & 1.8 & 4 & 36 & 0.9 & 0.7 \\ 1-7 & 86 & 1.7 & 5 & 37 & 0.8 & 0.7 \\ 1-7 & 110 & 2.8 & 5 & 36 & 1.6 & 0.9 \\ 1-7 & 9 & 0.3 & 5 & 4.2 & 0.2 & 0.1 \\ 1-7 & 59 & 2.3 & 4 & 29 & 1.1 & 0.6 \\ 1-7 & 70 & 2.4 & 5 & 33 & 1.1 & 0.5 \\ 1-7 & 70 & 0.9 & 3 & 28 & 0.7 & 0.5 \\ 1-7 & 70 & 1.9 & 4 & 27 & 1.0 & 0.9 \\ 1-7 & 120 & 1.7 & 5 & 33 & 1.3 & 0.8 \\ 1-7 & 39 & 1.7 & 4 & 36 & 0.8 & 0.7 \\ 1-7 & 62 & 1.6 & 4 & 38 & 0.6 & 0.8 \\ 1-7 & 370 & 0.5 & 6 & 63 & 0.5 & 0.4\end{array}$

Results less than analytical limit are indicated by a $(-)$. No entry indicates no analysis made. 
APPENDIX C

TABLE 1 (Continued)

CONCENTRATIONS OF RADIONUCLIDES IN MUSCLE OF

WHITEFISH TAKEN FROM THE COLUMBIA RIVER - 1966

$$
\text { Units of picocuries per gram }
$$

\begin{tabular}{|c|c|c|c|c|c|c|}
\hline Date & $32_{\mathrm{P}}$ & ${ }^{60} \mathrm{Co}$ & $4 \mathrm{O}_{\mathrm{K}}$ & ${ }^{65} \mathrm{Zn}$ & ${ }^{58} \mathrm{Co}$ & $137 \mathrm{cs}$ \\
\hline $\begin{array}{l}\text { Analytical } \\
\text { Limit }\end{array}$ & 1 & 0.2 & 1 & 0.2 & 0.2 & 0.1 \\
\hline
\end{tabular}

Hanford (Continued)

\begin{tabular}{|c|c|c|c|c|c|c|}
\hline $2-9$ & 92 & 9.0 & 10 & 85 & 4.3 & 1.7 \\
\hline $2-9$ & 25 & 2.1 & 3 & 28 & 1.2 & 0.6 \\
\hline $2-9$ & - & 3.1 & 3 & 19 & 0.9 & 0.6 \\
\hline $2-9$ & 13 & 0.5 & 3 & 18 & 0.2 & 0.2 \\
\hline $2-9$ & 34 & 1.7 & 3 & 31 & 1.0 & 0.7 \\
\hline $2-9$ & 18 & 1.2 & 3 & 22 & 0.7 & 0.3 \\
\hline $2-9$ & 26 & 1.1 & 3 & 25 & 0.6 & 0.5 \\
\hline $2-9$ & 10 & 1.0 & 4 & 5.1 & 0.6 & 0.9 \\
\hline $2-9$ & 31 & 2.3 & 3 & 32 & 1.3 & 0.6 \\
\hline $2-9$ & 40 & 1.6 & 5 & 36 & 1.0 & 0.9 \\
\hline $2-9$ & 43 & 2.1 & 3 & 39 & 0.9 & 0.5 \\
\hline $2-9$ & 32 & 1.8 & 4 & 32 & 0.6 & 0.4 \\
\hline $2-9$ & 30 & 1.4 & 4 & 31 & 0.3 & 0.5 \\
\hline $2-9$ & 27 & 1.6 & 4 & 36 & 0.4 & 0.4 \\
\hline $2-9$ & 38 & 1.1 & 5 & 28 & 0.2 & 0.3 \\
\hline $3-9$ & 20 & 2.8 & 5 & 30 & 1. & 0.9 \\
\hline $3-9$ & 26 & 1.9 & 4 & 22 & 0.9 & 0.4 \\
\hline $3-9$ & 22 & 2.2 & 5 & 28 & 1.2 & 0.8 \\
\hline $3-9$ & 7 & 2.3 & 3 & 28 & 1.1 & 0.9 \\
\hline $3-9$ & 28 & 2.1 & 4 & 27 & 1.1 & 0.8 \\
\hline $3-9$ & 34 & 2.4 & 4 & 38 & 1.2 & 1.0 \\
\hline $3-9$ & 61 & 1.8 & 4 & 40 & 0.5 & 0.5 \\
\hline $3-9$ & 14 & 1.1 & 4 & 20 & 0.4 & 0.5 \\
\hline $3-9$ & 53 & 0.4 & 6 & 19 & 0.6 & 0.2 \\
\hline $3-9$ & 7 & 0.5 & 4 & 20 & 0.3 & 0.6 \\
\hline $3-9$ & 1.7 & 2.2 & 5 & 40 & 1.1 & 0.9 \\
\hline $3-9$ & 57 & 2.6 & 4 & 38 & 0.5 & 1.0 \\
\hline $3-9$ & 68 & 0.5 & 4 & 26 & - & 0.3 \\
\hline $3-9$ & 25 & 2.4 & 5 & 21 & 1.2 & 0.9 \\
\hline $3-9$ & 46 & 1.3 & 5 & 26 & 0.3 & 0.5 \\
\hline $4-13$ & 67 & 2.9 & 5 & 25 & 1.5 & 0.9 \\
\hline $4-13$ & 130 & 0.6 & 5 & 20 & 0.4 & 0.7 \\
\hline $4-13$ & 81 & 1.7 & 5 & 30 & 0.5 & 0.6 \\
\hline $4-.13$ & 220 & 1.6 & 5 & 24 & 0.5 & 0.7 \\
\hline $4-13$ & 310 & 0.8 & 5 & 26 & 0.2 & 0.7 \\
\hline $4-13$ & 130 & 1.4 & 5 & 26 & 0.4 & 0.7 \\
\hline
\end{tabular}

Results less than analytical 1 imit are indicated by a (-). No entry indicates no analysis made. 
APPENDIX C

TABLE 1 (Cont inued)

CONCENTRATIONS OF RADIONUCLIDES IN MUSCLE OF

WHITEFISH TAKEN FROM THE COLUMBIA RIVER - 1966

Units of picocuries per gram

\begin{tabular}{|c|c|c|c|c|c|c|}
\hline Date & $32_{P}$ & ${ }^{60} \mathrm{Co}$ & ${ }_{4}^{4} \mathrm{~K}$ & $65 \mathrm{Zn}$ & ${ }^{58} \mathrm{Co}$ & ${ }^{137} \mathrm{C}$ \\
\hline $\begin{array}{l}\text { Analytical } \\
\text { Limit }\end{array}$ & 1 & 0.2 & 1 & 0.2 & 0.2 & 0.1 \\
\hline \multicolumn{7}{|c|}{ Hanford (Continued) } \\
\hline $5-13$ & 42 & 4.7 & 2 & 31 & 1.7 & 1.3 \\
\hline $5-13$ & 15 & 0.2 & 2 & .3 .6 & - & 0.1 \\
\hline $5-13$ & 110 & 3.4 & 3 & 26 & 1.4 & 1.1 \\
\hline 6.3 & 22 & 2.2 & 6 & 20 & 0.5 & 0.5 \\
\hline $6-3$ & 55 & 2.8 & 3 & 26 & 1.1 & 0.9 \\
\hline $6-3$ & 42 & 2.4 & 3 & 28 & 0.9 & 1.1 \\
\hline $6-3$ & 10 & 3.8 & 3 & 25 & 0.9 & 1.1 \\
\hline $6-29$ & 10 & 1.5 & 3 & 16 & 0.7 & 0.5 \\
\hline $6-29$ & 13 & 0.2 & 4 & 3.7 & 0.2 & 0.1 \\
\hline $6-29$ & 17 & 1.1 & 3 & 14 & 0.4 & 0.6 \\
\hline $6-29$ & 8 & 1.8 & 3 & 18 & 0.6 & 0.5 \\
\hline $6-29$ & 34 & 1.3 & 5 & 24 & 0.6 & 1.0 \\
\hline $6-29$ & 31 & 1.7 & 3 & 24 & 0.7 & 0.7 \\
\hline $6=29$ & 26 & 4.0 & 1 & 22 & 1.2 & 1.3 \\
\hline $6-29$ & 15 & 1.6 & 5 & 12 & 0.8 & 0.4 \\
\hline $6-29$ & 24 & 1.6 & 4 & 16 & 0.8 & 0.5 \\
\hline $6-29$ & 27 & 2.1 & 3 & 23 & 1.0 & 0.5 \\
\hline $6-29$ & 25 & 1.5 & 5 & 15 & 0.6 & 0.6 \\
\hline $6-29$ & 26 & 1.3 & 3 & 16 & 0.5 & 0.7 \\
\hline $6-29$ & 49 & 1.4 & 5 & 20 & 0.6 & 0.7 \\
\hline $6-29$ & 76 & 1.2 & 5 & 10 & 0.7 & 0.3 \\
\hline $6-29$ & 44 & 1.7 & 5 & 16 & 0.7 & 0.5 \\
\hline $7=21$ & 22 & 1.9 & 3 & 11 & 0.6 & 0.9 \\
\hline $7-21$ & 21 & 0.3 & 4 & 7.0 & 0.3 & 0.1 \\
\hline $7-21$ & 34 & 1.7 & 3 & 16 & 0.6 & 0.8 \\
\hline $8-31$ & 9 & 1.5 & 5 & 14 & 0.4 & 0.8 \\
\hline $8-31$ & 12 & 1.7 & 4 & 15 & 0.4 & 0.9 \\
\hline $8-31$ & 26 & 2.4 & 2 & 13 & 0.6 & 1.2 \\
\hline 8.31 & 18 & 1.6 & 5 & 15 & 0.4 & 0.9 \\
\hline $8-31$ & 8 & 1.7 & 5 & 13 & 0.3 & 0.9 \\
\hline $8+31$ & 5 & 1.8 & 3 & 7.8 & 0.6 & 0.9 \\
\hline $9 \cdot 28$ & 150 & 1.0 & 7 & 23 & 0.3 & 0.5 \\
\hline $9-28$ & 260 & 0.8 & 10 & 24 & 0.4 & 0.7 \\
\hline $9-28$ & 110 & 1.4 & 9 & 21 & 0.4 & 0.5 \\
\hline $9-28$ & 130 & 0.9 & 6 & 15 & 0.3 & 0.5 \\
\hline $9-28$ & 56 & 0.3 & 4 & 14 & - & 0.3 \\
\hline
\end{tabular}

Results less than analytical limit are indicated by a ( $)$. No entry indicates no analysis made. 
APPENDIX C

TABLE 1 (Continued)

CONCENTRATIONS OF RADIONUCLIDES IN MUSCLE OF

WHITEFISH TAKEN FROM THE COLUMBIA RIVER - 1966

Units of picocuries per gram

\begin{tabular}{|c|c|c|c|c|c|}
\hline Date & $32_{P}$ & ${ }^{60} \mathrm{Co}_{\mathrm{O}}$ & $40_{K}$ & $65 \mathrm{Zn}$ & ${ }_{\mathrm{Co}}^{58}$ \\
\hline Analytical & 1 & 0.2 & 1 & 0.2 & 0.2 \\
\hline
\end{tabular}

Hanford (Continued)

$\begin{array}{lrlllll}10-13 & 120 & 0.4 & 4 & 19 & 0.7 & 1.4 \\ 10-13 & 180 & 2.0 & 4 & 18 & 0.6 & 0.9 \\ 10-13 & 150 & 0.6 & 5 & 25 & 0.4 & 0.7 \\ 10-13 & 180 & 0.8 & 4 & 21 & 0.6 & 0.7 \\ 10-13 & 210 & 0.5 & 4 & 16 & 0.7 & 0.4 \\ 11-15 & 150 & 1.9 & 4 & 28 & 0.9 & 1.2 \\ 11-15 & 83 & 1.6 & 4 & 22 & 0.7 & 0.7 \\ 11-15 & 110 & 1.0 & 4 & 24 & 0.7 & 0.5 \\ 11-15 & 340 & 1.6 & 4 & 31 & 1.1 & 0.6 \\ 11-15 & 44 & 0.8 & 5 & 13 & 0.2 & 0.4 \\ 12-6 & 170 & 1.3 & 5 & 27 & 1.0 & 1.0 \\ 12-6 & 240 & 2.3 & 4 & 22 & 0.8 & 0.9 \\ 12-6 & 57 & 1.4 & 4 & 15 & 1.0 & 0.7 \\ 12-6 & 80 & 1.2 & 4 & 17 & 1.1 & 1.0 \\ 12-6 & 160 & 2.3 & 7 & 25 & 1.3 & 0.4 \\ 12-6 & 110 & 1.5 & 4 & 19 & 0.8 & 0.8 \\ 12-6 & 70 & 0.8 & 3 & 13 & 0.4 & 0.8 \\ 12-6 & 90 & 0.2 & 5 & 11 & 0.4 & 0.3 \\ 12-6 & 160 & 1.9 & 9 & 26 & 0.8 & 0.5\end{array}$

Ringold

\begin{tabular}{lllllll}
\hline $1-27$ & 63 & 2.6 & 3 & 34 & 1.1 & 0.5 \\
$1-27$ & 51 & 2.2 & 5 & 32 & 1.9 & 0.7 \\
$1-27$ & 59 & 2.4 & 5 & 28 & 1.6 & 0.6 \\
$1-27$ & 42 & 3.2 & 4 & 36 & 1.4 & 0.7 \\
$1-27$ & 57 & 2.0 & 4 & 34 & 0.8 & 0.4 \\
$1-27$ & 75 & 2.0 & 4 & 39 & 0.8 & 0.6 \\
$1-27$ & 69 & 1.6 & 4 & 38 & 0.7 & 0.7 \\
$1-27$ & 53 & 2.0 & 5 & 46 & 0.8 & 0.7 \\
$2-16$ & 32 & 2.2 & 3 & 30 & 1.2 & 0.9 \\
$2-16$ & 71 & 1.0 & 4 & 27 & 0.3 & 0.7 \\
$2-16$ & 40 & 2.6 & 4 & 31 & 0.7 & 1.0 \\
$2-16$ & 31 & 1.7 & 4 & 33 & 0.6 & 0.5 \\
$2-16$ & 61 & 1.8 & 4 & 28 & 0.6 & 0.9 \\
$2-16$ & 77 & 2.0 & 3 & 46 & 0.8 & 0.6 \\
$2-16$ & 300 & 1.2 & 4 & 49 & 0.2 & 0.7
\end{tabular}

Results less than analytical 1 imit are indicated by a (-). No entry indicates no analys is made. 


\section{APPENDIX C}

TABLE 1 (Continued)

CONCENTRATIONS OF RADIONUCLIDES IN MUSCLE OF WHITEFTSH IAKEN FROM THE COLUMBIA RIVER - 1966 Units of picocuries per gram

\begin{tabular}{|c|c|c|c|c|c|c|}
\hline Date & $32_{p}$ & ${ }^{60} \mathrm{Co}$ & $40_{K}$ & $65_{\mathrm{Zn}}$ & ${ }^{50} \mathrm{Ce}$ & ${ }^{137} \mathrm{Cs}$ \\
\hline $\begin{array}{l}\text { Analyt } \\
\text { Limit }\end{array}$ & 1 & 0.2 & 1 & 0.2 & 0.2 & 0.1 \\
\hline \multicolumn{7}{|c|}{ Ringold (Continued) } \\
\hline $2-28$ & 35 & 1.9 & 7 & 27 & 0.8 & 1.1 \\
\hline $2-28$ & 280 & - & 10 & 29 & - & 0.2 \\
\hline $2-28$ & 320 & 0.4 & 7 & 40 & 0.2 & 0.6 \\
\hline $2-28$ & 340 & & 11 & 52 & & 0.6 \\
\hline $3-18$ & 420 & 1.5 & 7 & 49 & 0.2 & 0.9 \\
\hline $3-18$ & 580 & 0.9 & 6 & 70 & - & 0.3 \\
\hline $3-22$ & 64 & 1.8 & 6 & 22 & 0.3 & 1.1 \\
\hline $3-22$ & 270 & 1.3 & 8 & 41 & 0.4 & 0.6 \\
\hline $3-22$ & 250 & 1.3 & 8 & 37 & 0.4 & 1.1 \\
\hline $4-7$ & 100 & 2.2 & 7 & 46 & 1.0 & 0.5 \\
\hline $4-7$ & 320 & 1.5 & 13 & 47 & - & 0.4 \\
\hline 407 & 53 & $\infty$ & - & 8.4 & - & - \\
\hline 407 & 17 & 1.0 & 5 & 37 & - & - \\
\hline 4.7 & 200 & 1.3 & 6 & 18 & - & 0.4 \\
\hline $4-7$ & 33 & 0.9 & 5 & 29 & - & 0.3 \\
\hline $4-7$ & 110 & 1.8 & 4 & 26 & 0.7 & 0.8 \\
\hline $4-7$ & 71 & 2.2 & 5 & 45 & 1.1 & 0.6 \\
\hline $4-7$ & 29 & 7.2 & 5 & 23 & 1.6 & 1.0 \\
\hline $4-7$ & 47 & 2.9 & 3 & 32 & 1.1 & 0.6 \\
\hline $4-27$ & 230 & 0.7 & 4 & 14 & 0.3 & 0.1 \\
\hline $4-27$ & 240 & 2.7 & 5 & 34 & 0.9 & 1.0 \\
\hline 4.27 & 210 & 2.1 & 5 & 26 & 1.5 & 2.2 \\
\hline $4 .-27$ & 570 & 0.8 & 5 & 32 & 0.2 & 0.6 \\
\hline- & 1.90 & 0.8 & 7 & 10 & 0.2 & 0.3 \\
\hline - & 300 & 0.6 & 4 & 13 & 0.4 & 0.3 \\
\hline - & 210 & 2.1 & 4 & 29 & 0.7 & 1.1 \\
\hline$=$ & 500 & 0.7 & 3 & 41 & $=$ & 0.5 \\
\hline- & 290 & 1.4 & 5 & 30 & 0.5 & 0.8 \\
\hline$=$ & 720 & - & 5 & 35 & 0.2 & 0.4 \\
\hline- & 170 & 2.2 & 5 & 24 & 0.9 & 1.0 \\
\hline- & 280 & 0.5 & 4 & 11 & 0.2 & 0.2 \\
\hline- & 350 & 1.0 & 5 & 32 & 0.5 & 0.8 \\
\hline- & 490 & - & 6 & 17 & - & - \\
\hline $5-23$ & 86 & 4.0 & 3 & 21 & 1.5 & 1.1 \\
\hline $5-23$ & 67 & 4.2 & 2 & 22 & 1.7 & 0.8 \\
\hline
\end{tabular}

Results less than analytical limit are indicated by $a(-)$. No entry indicates no analysis made. 
APPENDIX C

TABLE 1 (Continued)

CONCENTRATIONS OF RADIONUCLIDES IN MUSCLE OF WHITEFISH TAKEN FROM THE COLUMBIA RIVER - 1966

\begin{tabular}{|c|c|c|c|c|c|c|}
\hline Date & $32_{P}$ & ${ }^{60} \mathrm{Co}$ & $40_{K}$ & ${ }^{65} \mathrm{Zn}$ & ${ }^{58} \mathrm{Co}$ & ${ }^{137} \mathrm{Cs}$ \\
\hline $\begin{array}{l}\text { Analytical } \\
\text { Limit }\end{array}$ & 1 & 0.2 & 1 & 0.2 & 0.2 & 0.1 \\
\hline \multicolumn{7}{|c|}{ Ringo1d (Continued) } \\
\hline $5-23$ & 51 & 1.1 & 4 & 16 & 0.4 & 0.4 \\
\hline $5-23$ & 46 & 0.7 & 5 & 8.9 & 0.4 & 0.2 \\
\hline $5-23$ & 10 & 0.2 & 3 & 6.8 & - & 0.1 \\
\hline $5-23$ & 150 & 1.3 & 3 & 24 & 0.2 & 0.7 \\
\hline $5-23$ & 130 & 1.4 & 5 & 23 & 0.2 & 0.9 \\
\hline $5-23$ & 28 & 1.3 & 3 & 7.4 & 0.3 & 0.2 \\
\hline $5-23$ & 140 & 3.4 & 2 & 29 & 1.0 & 1.1 \\
\hline $5-23$ & 58 & 1.6 & 5 & 22 & 0.5 & 1.3 \\
\hline $5-23$ & 9 & 0.2 & 3 & 3.3 & - & - \\
\hline $5-23$ & 74 & 2.1 & 4 & 11 & 0.8 & 0.4 \\
\hline $5-23$ & 96 & 1.3 & 5 & 20 & 0.4 & 1.2 \\
\hline $5-23$ & 190 & 1.1 & 3 & 18 & 0.4 & 0.3 \\
\hline $5-23$ & 130 & 2.8 & 4 & 26 & 1.0 & 1.0 \\
\hline $5-23$ & 74 & 1.5 & 3 & 15 & 0.4 & 1.0 \\
\hline $6-15$ & 8 & 0.7 & 4 & 4.2 & 0.2 & 0.3 \\
\hline $6-15$ & - & 0.2 & 4 & 1.7 & $\infty$ & - \\
\hline $6-1.5$ & 60 & 1.9 & 4 & 27 & 0.7 & 0.9 \\
\hline $6-15$ & 120 & 1.4 & 3 & 29 & 0.3 & 0.9 \\
\hline $6-15$ & 24 & 1.6 & 4 & 20 & 0.6 & 1.0 \\
\hline $6-15$ & 81 & 0.2 & 4 & 4.9 & - & 0.1 \\
\hline $6-27$ & 33 & 2.7 & 4 & 18 & 0.9 & 1.1 \\
\hline $6-27$ & 22 & 3.0 & 5 & 22 & 0.8 & 1.3 \\
\hline $6-27$ & 35 & 3.2 & 4 & 20 & 1.1 & 1.1 . \\
\hline $6-27$ & 16 & 0.5 & 4 & 8.0 & 0.2 & - \\
\hline $6-27$ & 23 & 2.6 & 4 & 23 & 0.9 & 1.2 \\
\hline $6-27$ & 38 & 2.6 & 5 & 23 & 1.3 & 1.0 \\
\hline $6-27$ & 24 & 2.2 & 4 & 27 & 0.7 & 0.9 \\
\hline $6-27$ & 25 & 0.4 & 6 & 8.6 & 0.5 & 0.3 \\
\hline $6-27$ & 120 & 0.8 & 9 & 26 & 0.3 & 0.8 \\
\hline $6-27$ & 150 & 2.0 & 7 & 23 & 1.0 & 0.8 \\
\hline $6-27$ & 67 & 1.6 & 6 & 24 & 0.6 & 1.0 \\
\hline $6-27$ & 42 & 1.3 & 5 & 25 & 0.2 & 1.2 \\
\hline $6-27$ & 41 & 1.7 & 7 & 22 & 0.6 & 1.2 \\
\hline $6-27$ & 150 & 1.5 & 6 & 19 & 0.7 & 0.1 \\
\hline $7-14$ & 130 & & 3 & 27 & 0.3 & 0.7 \\
\hline $7-14$ & 8 & 0.3 & 4 & 3.0 & 0.3 & 0.2 \\
\hline
\end{tabular}

Results less than analytical limit are indicated by a (-). No entry indicates no analysis made. 
APPENDIX C

TABLE 1 (Continued)

CONCENTRATIONS OF RADIONUCLIDES IN MUSCLE OF WHITEFISH TAKEN FROM THE COLUMBIA RIVER - 1966

Units of picocuries per gram

\begin{tabular}{|c|c|c|c|c|c|c|}
\hline Date & $32_{\mathrm{P}}$ & ${ }^{60} \mathrm{Co}$ & ${ }^{4 O_{K}}$ & ${ }^{65} \mathrm{Zn}$ & ${ }^{58} \mathrm{Co}$ & ${ }^{137} \mathrm{Cs}_{\mathrm{S}}$ \\
\hline Analytical & Limit 1 & 0.2 & 1 & 0.2 & 0.2 & 0.1 \\
\hline \multicolumn{7}{|c|}{ Ringold (Continued) } \\
\hline $7-14$ & 37 & 2.1 & 3 & 21 & 1.3 & 0.9 \\
\hline $7-14$ & 10 & 0.3 & 3 & 4.7 & 0.2 & 0.2 \\
\hline $7-14$ & 14 & 2.2 & 4 & 23 & 0.6 & 1.1 \\
\hline $7-14$ & 17 & 0.5 & 4 & 6.4 & 0.5 & - \\
\hline $7-14$ & 11 & 0.5 & 2 & 4.2 & 0.6 & 0.1 \\
\hline $7-14$ & 28 & 0.4 & 4 & 6.6 & 0.7 & - \\
\hline $7-14$ & 12 & 2.6 & 3 & 17 & 0.6 & 1.3 \\
\hline $7-14$ & 180 & 0.4 & 3 & 11 & 0.3 & 0.3 \\
\hline $7-14$ & 37 & 2.2 & 3 & 21 & 0.9 & 1.0 \\
\hline $7-14$ & 17 & 1.9 & 3 & 19 & 1.2 & 0.4 \\
\hline $7-14$ & 28 & 2.3 & 4 & 25 & 0.4 & 1.2 \\
\hline $7-14$ & 34 & 3.6 & 3 & 28 & 1.0 & 1.4 \\
\hline $7-15$ & 20 & 1.2 & 5 & 30 & 0.2 & 0.8 \\
\hline $7-15$ & 18 & 2.5 & 3 & 26 & 0.9 & 0.9 \\
\hline $7-15$ & 85 & 0.9 & 4 & 42 & 0.7 & 0.8 \\
\hline $7-15$ & 32 & 2.0 & 4 & 27 & 0.5 & 1.5 \\
\hline $7-15$ & 85 & 0.9 & 4 & 28 & - & 1.1 \\
\hline $7-15$ & 13 & 0.2 & 3 & 19 & 0.6 & 0.9 \\
\hline $7-15$ & 21 & 0.4 & 3 & 4.6 & 0.3 & 1.2 \\
\hline $7-15$ & 93 & 2.1 & 4 & 37 & 0.8 & 0.9 \\
\hline $7-15$ & 28 & 0.7 & 4 & 6.4 & 0.7 & 0.3 \\
\hline $7-15$ & 10 & 3.7 & 4 & 18 & 0.8 & 1.2 \\
\hline $7-15$ & 27 & 1.4 & 5 & 28 & 0.7 & 1.4 \\
\hline $7-15$ & 140 & 1.0 & 4 & 37 & 0.6 & 1.1 \\
\hline $7-15$ & 830 & 0.4 & 3 & 52 & $=$ & 0.3 \\
\hline $7-15$ & 250 & 0.5 & 3 & 19 & 0.3 & 0.4 \\
\hline $7-15$ & 80 & 0.7 & 3 & 16 & 0.4 & 0.3 \\
\hline $7-15$ & 31 & 1.7 & 6 & 20 & 0.7 & 0.6 \\
\hline $7-15$ & 33 & 1.2 & 5 & 21 & 0.5 & 0.9 \\
\hline $7-19$ & 4 & 4.2 & 3 & 22 & 1.1 & 1.1 \\
\hline $7-19$ & 13 & 1.8 & 4 & 22 & 0.6 & 1.1 \\
\hline $7-19$ & 9 & 4.2 & 3 & 25 & 1.6 & 1.3 \\
\hline $7-19$ & 17 & 1.8 & 2 & 20 & 0.8 & 1.4 \\
\hline $7-19$ & 62 & 0.5 & 5 & 16 & 0.6 & 0.5 \\
\hline $7-19$ & 15 & 0.9 & 4 & 12 & 0.5 & 0.4 \\
\hline $7-19$ & 20 & 0.4 & 3 & 7.0 & 0.5 & 0.1 \\
\hline
\end{tabular}

Results less than analytical limit are indicated by a $(-)$. No entry indicates no analys is made. 
APPENDIX C

TABLE 1 (Continued)

CONCENTRATIONS OF RADIONUCLIDES IN MUSCLE OF WHITEFISH TAKEN FROM THE COLUMBIA RIVER - 1966

\begin{tabular}{|c|c|c|c|c|c|c|}
\hline Date & $32 \mathrm{p}$ & ${ }^{60} \mathrm{Co}$ & $4 \mathrm{O}_{\mathrm{K}}$ & $65 \mathrm{Zn}$ & ${ }^{58} \mathrm{Co}$ & $137 \mathrm{Cs}$ \\
\hline $\begin{array}{l}\text { Analytical } \\
\text { Limit }\end{array}$ & 1 & 0.2 & 1 & 0.2 & 0.2 & 0.1 \\
\hline \multicolumn{7}{|c|}{ Ringold (Cont inued) } \\
\hline $7-19$ & 21 & 0.3 & 4 & 5.4 & 0.3 & - \\
\hline $7-19$ & 5 & 3.3 & 2 & 25 & 1.2 & 1.4 \\
\hline $7-19$ & 10 & 0.4 & 4 & 4.2 & 0.3 & - \\
\hline $7-19$ & 48 & 0.5 & 3 & 6.8 & 0.4 & 0.3 \\
\hline $7-19$ & 15 & 0.9 & 4 & 8.3 & 0.6 & 0.1 \\
\hline $7-19$ & 29 & 1.6 & 4 & 12 & 0.7 & 0.6 \\
\hline $7-19$ & 140 & 0.7 & 4 & 24 & 0.3 & 0.5 \\
\hline $7-19$ & 20 & 1.0 & 4 & 8.2 & 0.9 & 0.3 \\
\hline $7-19$ & 17 & 1.1 & 2 & 7.5 & 0.7 & 0.4 \\
\hline $7-19$ & 17 & 0.4 & 3 & 5.7 & 0.6 & - \\
\hline $7-19$ & 77 & 1.7 & 4 & 32 & 1.3 & 0.9 \\
\hline $7-19$ & - & 1.1 & 3 & 14 & 0.6 & 0.4 \\
\hline $7-19$ & 15 & 0.8 & 4 & 8.0 & 0.6 & 0.2 \\
\hline $7-19$ & 37 & 1.0 & 4 & 22 & 0.5 & 1.0 \\
\hline $7-19$ & 22 & 1.3 & 3 & 19 & 0.6 & 1.1 \\
\hline $7-19$ & 120 & 1.1 & 5 & 21 & 0.3 & 0.6 \\
\hline $7-19$ & 160 & 0.2 & 4 & 14 & 0.2 & 0.2 \\
\hline $7-19$ & 28 & 1.4 & 4 & 17 & 0.4 & 0.7 \\
\hline $7-19$ & 23 & 2.7 & 4 & 23 & 0.7 & 1.0 \\
\hline $7-19$ & 7 & 0.8 & 3 & 16 & 0.2 & 0.3 \\
\hline $7-19$ & 29 & 0.4 & 4 & 7.6 & 0.4 & 0.1 \\
\hline $7-19$ & 19 & 1.1 & 3 & 13 & 0.6 & 0.5 \\
\hline $7-19$ & 140 & 0.8 & 3 & 19 & 0.2 & 0.3 \\
\hline $7-19$ & 9 & 1.2 & 5 & 13 & 0.6 & 1.0 \\
\hline $7-19$ & 6 & 0.2 & 3 & 2.6 & 0.2 & 0.1 \\
\hline $7-29$ & 9 & 0.3 & 3 & 3.1 & 0.2 & - \\
\hline $7-29$ & 12 & 0.7 & 3 & 8.7 & 0.4 & 0.2 \\
\hline $7-29$ & 16 & 1.1 & 3 & 9.0 & 0.6 & 0.3 \\
\hline $7-29$ & 22 & 0.9 & 2 & 9.1 & 0.4 & 0.2 \\
\hline $7-29$ & 46 & 0.7 & 4 & 13 & 0.3 & 0.4 \\
\hline $7-29$ & 24 & 0.4 & 3 & 6.7 & 0.2 & 0.2 \\
\hline $7-29$ & 24 & 2.0 & 3 & 26 & 0.8 & 1.0 \\
\hline $7-29$ & 28 & 1.7 & 3 & 25 & 0.7 & 0.9 \\
\hline $7-29$ & 58 & 1.9 & 3 & 29 & 0.6 & 1.0 \\
\hline $7-29$ & 11 & 0.4 & 2 & 4.8 & 0.5 & 0.1 \\
\hline $7-29$ & 35 & 1.1 & 3 & 9.3 & 0.7 & 0.2 \\
\hline
\end{tabular}

Results less than analytical limit are indicated by $a(-)$. No entry indicates no analys is made. 


\section{APPENDIX C \\ TABLE 1 (Continued)}

CONCENTRATIONS OF RADIONUCLIDES IN MUSCLE OF WHITEFISH TAKEN FROM THE COLUMBIA RIVER - 1966

\begin{tabular}{|c|c|c|c|c|c|c|}
\hline Date & $32 \mathrm{P}$ & ${ }^{60} \mathrm{Co}$ & $4 \mathrm{O}_{\mathrm{K}}$ & ${ }^{65} \mathrm{Zn}$ & ${ }^{58} \mathrm{Co}$ & $137_{\mathrm{C}}$ \\
\hline $\begin{array}{l}\text { Analytical } \\
\text { Limit }\end{array}$ & 1 & 0.2 & 1 & 0.2 & 0.2 & 0.1 \\
\hline \multicolumn{7}{|c|}{ Ringold (Cont inued) } \\
\hline $7-29$ & 34 & 1.4 & 4 & 18 & 0.8 & 0.5 \\
\hline $7-29$ & 28 & 1.7 & 5 & 22 & 0.5 & 1.0 \\
\hline $7-29$ & 51 & 1.6 & 4 & 18 & 0.9 & 0.7 \\
\hline $7-29$ & 87 & 1.0 & 3 & 24 & 0.4 & 0.6 \\
\hline $7-29$ & 91 & 0.4 & 5 & 11 & - & 0.2 \\
\hline $8-4$ & 9 & 1.5 & 5 & 20 & 0.6 & 1.0 \\
\hline $8-4$ & 3 & & 3 & 3.2 & - & - \\
\hline $8-4$ & 8 & 0.2 & 3 & 4.8 & - & 0.1 \\
\hline $8-4$ & 53 & 0.5 & 4 & 14 & 0.3 & 0.4 \\
\hline $8-4$ & 89 & 0.8 & 4 & 40 & 0.4 & 0.7 \\
\hline $8-4$ & 10 & 0.8 & 4 & 9.8 & 0.4 & 0.3 \\
\hline $8-4$ & 12 & 2.7 & 4 & 21 & 0.7 & 1.3 \\
\hline $8-4$ & 10 & 1.5 & 4 & 19 & 0.3 & 1.3 \\
\hline $8-4$ & 88 & 0.6 & 3 & 38 & 0.2 & 0.3 \\
\hline 8.4 & 17 & 0.8 & 3 & 13 & 0.5 & 0.4 \\
\hline $8-12$ & 8 & 2.2 & 4 & 20 & 0.6 & 0.8 \\
\hline $8-12$ & 100 & 0.5 & 5 & 32 & 0.2 & 0.6 \\
\hline $8-12$ & 34 & 0.4 & 5 & 13 & - & 0.2 \\
\hline $8-12$ & 21 & 1.1 & 3 & 15 & 0.5 & 0.4 \\
\hline $8 \propto 12$ & 24 & 1.1 & 4 & 21 & 0.2 & 0.6 \\
\hline $8-12$ & 15 & 1.9 & 4 & 19 & 0.3 & 0.9 \\
\hline $8-12$ & 10 & 0.5 & 4 & 4.1 & 0.4 & 0.2 \\
\hline $8-12$ & 16 & 2.1 & 5 & 20 & 0.5 & 0.8 \\
\hline $8-12$ & 5 & 1.3 & 4 & 20 & 0.3 & 2.0 \\
\hline $8-i 2$ & 20 & 0.4 & 3 & 9.8 & 0.5 & 0.2 \\
\hline $8-19$ & 7 & 1.0 & 4 & 12 & 0.3 & 0.6 \\
\hline $8=19$ & 2. & 1.3 & 3 & 15 & 0.4 & 0.7 \\
\hline $8-19$ & 9 & 0.3 & 3 & 5.8 & 0.2 & 0.2 \\
\hline $8-19$ & 5 & 2.0 & 4 & 30 & 0.2 & 1.1 \\
\hline $8-19$ & 3 & 2.9 & 4 & 22 & 0.6 & 1.4 \\
\hline $8 \cdot 19$ & 13 & 3.1 & 4 & 19 & 0.9 & 1.1 \\
\hline $8-19$ & 17 & 1.5 & 3 & 18 & 0.3 & 0.8 \\
\hline $8-19$ & 10 & 0.9 & 3 & 18 & 0.2 & 0.2 \\
\hline $8-26$ & 6 & 0.4 & 4 & 5.8 & 0.2 & 0.3 \\
\hline $8-26$ & 3 & 1.8 & 5 & 14 & 0.6 & 1.1 \\
\hline
\end{tabular}

Results less than analytical 1 imit are indicated by a $(-)$. No entry indicates no analysis made. 
APPENDIX C

TABLE 1 (Continued)

CONCENTRATIONS OF RADIONUCLIDES IN MUSCLE OF

WHITEFISH TAKEN FROM THE COLUMBIA RIVER - 1966

Date

$32 \mathrm{p}$

Analytical 1

Limit

$\underline{\text { Ringold (Continued) }}$

\begin{tabular}{|c|c|c|}
\hline $8-26$ & 15 & 1.5 \\
\hline $8-26$ & 4 & 0.7 \\
\hline $8-26$ & 32 & 0.3 \\
\hline $8-26$ & 10 & 1.3 \\
\hline $8-26$ & 12 & 0.5 \\
\hline $8-26$ & 72 & 1.0 \\
\hline $8-26$ & 15 & 0.4 \\
\hline $8-26$ & 14 & 1.9 \\
\hline $9-1$ & 19 & 0.4 \\
\hline $9-1$ & 17 & 1.7 \\
\hline $9-1$ & 23 & 1.8 \\
\hline $9-1$ & 8 & 1.2 \\
\hline $9-1$ & 14 & 0.6 \\
\hline $9-1$ & 11 & 1.5 \\
\hline $9-1$ & 14 & 1.7 \\
\hline $9-1$ & 20 & 2.5 \\
\hline $9-1$ & 27 & 0.6 \\
\hline $9-1$ & 14 & 1.0 \\
\hline $9-1$ & 16 & 0.6 \\
\hline $9-6$ & 21 & 2.1 \\
\hline $9-6$ & 21 & 0.6 \\
\hline $9-6$ & 34 & 0.6 \\
\hline $9-6$ & 14 & 0.7 \\
\hline $9-6$ & 48 & 0.6 \\
\hline $9-6$ & 23 & 1.5 \\
\hline $9-6$ & 33 & 1.0 \\
\hline $9-6$ & 15 & 0.6 \\
\hline $9-14$ & 37 & 1.5 \\
\hline $9-14$ & 60 & 0.6 \\
\hline $9-14$ & 14 & 0.9 \\
\hline $9-14$ & 66 & 0.8 \\
\hline $9-14$ & 63 & 0.8 \\
\hline $9-14$ & 44 & 1.9 \\
\hline $9-14$ & 29 & 0.8 \\
\hline $9-14$ & 79 & 1.2 \\
\hline $9-14$ & 150 & 1.5 \\
\hline $9-14$ & 68 & 0.6 \\
\hline
\end{tabular}

0.2

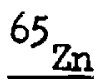

58

Co

0.2
$1 \quad 0.2$
137

0.1
$\mathrm{Cs}$

17

17

5.8

7.2

9.8

8.8

29

6.7

19

8.5

18

14

8.7

13

11

17

19

16

19

9.3

15

12

$$
8.2
$$

8.3

5.6

14

12

6.6

8.3

6.5

13

8.8

11

14

7.7

11

17

14
0.5

0.4

0.6

0.4

0.2

0.3

0.2

0.5

0.2

0.4

0.7

0.6

0.3

0.4

0.2

0.5

0.4

-

0.4

0.6

0.3

0.4

0.3

0.2

0.4

0.3

0.3

0.3

0.4

- 4

0.4

0.4

0.5

0.3

0.3

-
0.6

0.3

0.2

0.5

0.3

0.3

0.1

0.7

0.3

0.7

0.6

0.3

0.3

0.2

0.7

0.8

0.4

0.4

0.2

0.9

0.3

0.3

0.3

0.4

0.8

1.0

0.4

0.7

0.2

0.7

0.4

0.9

1.1

0.5

1.1

1.0

0.8

Results less than analytical limit are indicated by $a(-)$. No entry indicates no analysis made. 
APPENDIX C

TABLE 1 (Continued)

CONCENTRATIONS OF RADIONUCLIDES IN MUSCLE OF WHITEFISH TAKEN FROM THE COLUMBIA RIVER - 1966

Units of picocuries per gram

\begin{tabular}{|c|c|c|c|c|c|c|}
\hline Date & $32_{\mathrm{P}}$ & $60_{\mathrm{CO}}$ & $4 \mathrm{O}_{\mathrm{K}}$ & ${ }^{65} \mathrm{Zn}$ & ${ }^{58} \mathrm{Co}$ & $137 \mathrm{Cs}$ \\
\hline $\begin{array}{l}\text { Analytical } \\
\text { Limit }\end{array}$ & 1 & 0.2 & 1 & 0.2 & 0.2 & 0.1 \\
\hline
\end{tabular}

Ringold (Continued)

\begin{tabular}{|c|c|c|c|c|c|c|}
\hline 9.20 & 120 & 1.5 & 5 & 20 & 0.2 & 0.9 \\
\hline $9-20$ & 290 & 0.9 & 5 & 17 & 0.4 & 0.4 \\
\hline $9-20$ & 88 & 0.9 & 5 & 13 & 0.4 & 0.5 \\
\hline $9-20$ & 160 & 0.6 & 6 & 20 & $\infty$ & 1.1 \\
\hline $9-20$ & 1.70 & 1.0 & 4 & 12 & 0.4 & 0.6 \\
\hline $9-20$ & 77 & 0.7 & 8 & 17 & 0.2 & 0.7 \\
\hline $9-20$ & 75 & 1.3 & 4 & 17 & 0.4 & 0.9 \\
\hline $9-20$ & 66 & 0.4 & 4 & 11 & 0.2 & 0.4 \\
\hline $9-20$ & 48 & 0.4 & 4 & 5.6 & 0.2 & 0.2 \\
\hline $9-20$ & 170 & 2.1 & 5 & 17 & 0.7 & 0.7 \\
\hline $9-20$ & 52 & 0.7 & 5 & 13 & 0.3 & 0.7 \\
\hline $9-20$ & 100 & 0.9 & 5 & 19 & 0.2 & 0.7 \\
\hline $9-20$ & 99 & 2.4 & 5 & 18 & 0.6 & 1.1 \\
\hline $10-3$ & 280 & 2.4 & 5 & 18 & 0.7 & 1.1 \\
\hline $10-3$ & 520 & 1.0 & 5 & 24 & 0.5 & 0.8 \\
\hline $10-3$ & 170 & 9.8 & 5 & 22 & 0.8 & 0.5 \\
\hline $10-3$ & 340 & 1.3 & 3 & 39 & 0.7 & 0.8 \\
\hline $10-3$ & 410 & 1.7 & 5 & 25 & 0.8 & 0.6 \\
\hline $10-3$ & 390 & 1.6 & 4 & 18 & 0.6 & 0.6 \\
\hline $10-3$ & 420 & 0.7 & 4 & 17 & 0.3 & 0.6 \\
\hline $10-3$ & 270 & 1.1 & 2 & 22 & 0.9 & 0.5 \\
\hline $10-3$ & 180 & 1.3 & 4 & 23 & 0.6 & 0.7 \\
\hline $10-3$ & 380 & 1.1 & 5 & 21 & 0.8 & 0.6 \\
\hline $10-3$ & 500 & 1.01 & 5 & 29 & 0.9 & 0.6 \\
\hline $10-31$ & 140 & 1.7 & 5 & 18 & 0.4 & 0.9 \\
\hline $10-31$ & 230 & 1.2 & 5 & 20 & 0.4 & 1.1 \\
\hline $10-31$ & 150 & 0.8 & 5 & 22 & 0.7 & 0.6 \\
\hline $10 \cdot 31$ & 49 & 0.3 & 5 & 14 & $m$ & 0.2 \\
\hline $10 \cdot 31$ & 480 & 1.01 & 5 & 28 & 0.8 & 0.5 \\
\hline $10-31$ & 93 & 0.4 & 8 & 15 & - & 0.2 \\
\hline $10-31$ & 380 & 0.7 & 7 & 32 & 0.4 & 0.6 \\
\hline $10-31$ & 300 & 1.6 & 5 & 25 & 1.0 & 0.9 \\
\hline $12-8$ & 400 & 1.8 & 5 & 46 & 1.0 & 0.4 \\
\hline $12-8$ & 310 & 1.5 & 4 & 29 & 0.8 & 0.5 \\
\hline $12-8$ & 330 & 0.5 & 6 & 41 & $=$ & 0.1 \\
\hline $12-8$ & 170 & 4.2 & 6 & 22 & 1.2 & 1.2 \\
\hline $12=8$ & 360 & 1.5 & 9 & 34 & 0.4 & 0.4 \\
\hline $12-8$ & 25 & 1.0 & 4 & 20 & $=$ & 0.9 \\
\hline $12-8$ & 180 & 2.8 & 5 & 42 & 1.5 & 0.6 \\
\hline
\end{tabular}

Results less than analyticail limit are indicated by a ( $)$. No ertry indicates no analysis made. 
APPENDIX C

TABLE 1 (Continued)

CONCENTRATIONS OF RADIONUCLIDES IN MUSCLE OF WHITEFISH TAKEN FROM THE COLUMBIA RIVER - 1966

$$
\text { Units of picocuries per gram }
$$

\begin{tabular}{|c|c|c|c|c|c|c|}
\hline Date & $32_{\mathrm{P}}$ & ${ }^{60} \mathrm{Co}$ & $4 \mathrm{O}_{\mathrm{K}}$ & ${ }^{65} \mathrm{Zn}$ & ${ }^{58} \mathrm{Co}$ & $137_{\mathrm{Cs}}$ \\
\hline Analytical & 1 & 0.2 & 1 & 0.2 & 0.2 & 0.1 \\
\hline
\end{tabular}

$\underline{\text { Richland }}$

\begin{tabular}{|c|c|c|c|c|c|c|}
\hline $1-25$ & 25 & 2.2 & 4 & 34 & 1.3 & 0.7 \\
\hline $1-25$ & 55 & 2.5 & 4 & 48 & 1.3 & 0.6 \\
\hline $1-25$ & 110 & 2.9 & 3 & 63 & 1.6 & 0.1 \\
\hline $1-25$ & 48 & 2.4 & 5 & 48 & 1.4 & 0.5 \\
\hline $1-25$ & 55 & 3.3 & 5 & 31 & 1.7 & 0.4 \\
\hline $1-25$ & 81 & 3.1 & 5 & 51 & 1.5 & 0.3 \\
\hline $1-25$ & 90 & 2.2 & 6 & 55 & 1.4 & 0.3 \\
\hline $1-25$ & 44 & 2.7 & 3 & 39 & 1.3 & 0.6 \\
\hline $4-22$ & 100 & 2.7 & 4 & 35 & 1.7 & 0.8 \\
\hline $4-22$ & 5 & 0.2 & 3 & 16 & - & - \\
\hline $4-22$ & 300 & 2.0 & 4 & 36 & 0.8 & 0.8 \\
\hline $4-26$ & 56 & 3.8 & 2 & 30 & 1.8 & 1.0 \\
\hline $4-26$ & 460 & 1.0 & 4 & 33 & 0.5 & 0.4 \\
\hline $4-26$ & 180 & 4.3 & 3 & 30 & 2.1 & 1.1 \\
\hline $5-23$ & 17 & 0.5 & 3 & 6.3 & 0.2 & 0.1 \\
\hline $5-23$ & 1 & 0.2 & 3 & 0.8 & - & 0.1 \\
\hline $5-23$ & 33 & 0.6 & 3 & 17 & 0.2 & 1.3 \\
\hline $5-23$ & 21 & 0.2 & 4 & 5.5 & - & 0.1 \\
\hline $5-23$ & 110 & 3.1 & 4 & 31 & 1.4 & 0.8 \\
\hline $5-31$ & 52 & 2.7 & 3 & 30 & 1.0 & 0.8 \\
\hline $5-31$ & 52 & 1.9 & 4 & 25 & 0.8 & 0.8 \\
\hline $5-31$ & 42 & 1.5 & 4 & 19 & 0.5 & 0.5 \\
\hline 5031 & 59 & 2.1 & 4 & 31 & 0.6 & 1.0 \\
\hline $5-31$ & 120 & 2.2 & 3 & 32 & 1.1 & 0.5 \\
\hline $6=27$ & 14 & 1.4 & 5 & 22 & 0.6 & 1.1 \\
\hline $6-27$ & 18 & 0.4 & 2 & 4.5 & 0.3 & - \\
\hline $6-27$ & 32 & 0.5 & 4 & 7.3 & 0.2 & 0.1 \\
\hline $6-27$ & 28 & 3.7 & 4 & 19 & 1.3 & 0.8 \\
\hline $6-27$ & 15 & 0.2 & 5 & 4.4 & 0.2 & - \\
\hline $6-27$ & 34 & 3.0 & 3 & 22 & 1.6 & 0.6 \\
\hline $6-27$ & 29 & 0.3 & 5 & 7.6 & 0.2 & 0.1 \\
\hline $6-28$ & 14 & 3.3 & 4 & 22 & 1.0 & 1.0 \\
\hline $6-28$ & 13 & 2.1 & 4 & 18 & 0.7 & 0.8 \\
\hline $6-28$ & 83 & 1.2 & 3 & 20 & 0.9 & 0.4 \\
\hline $6-30$ & 17 & 0.2 & 3 & 3.6 & 0.2 & $=$ \\
\hline $6-30$ & 18 & 0.3 & 3 & 6.1 & 0.2 & - \\
\hline $6-30$ & 34 & 2.0 & 5 & 24 & 0.8 & 0.8 \\
\hline
\end{tabular}

Results less than analytical limit are indicated by a (-). No entry indicates no analysis made. 
APPENDIX C

TABLE 1 (Continued)

CONCENTRATIONS OF RADIONUCLIDES IN MUSCLE OF WHITEFISH TAKEN FROM THE COLUMBIA RIVER - 1966

$$
\text { Units of picocuries per gram }
$$

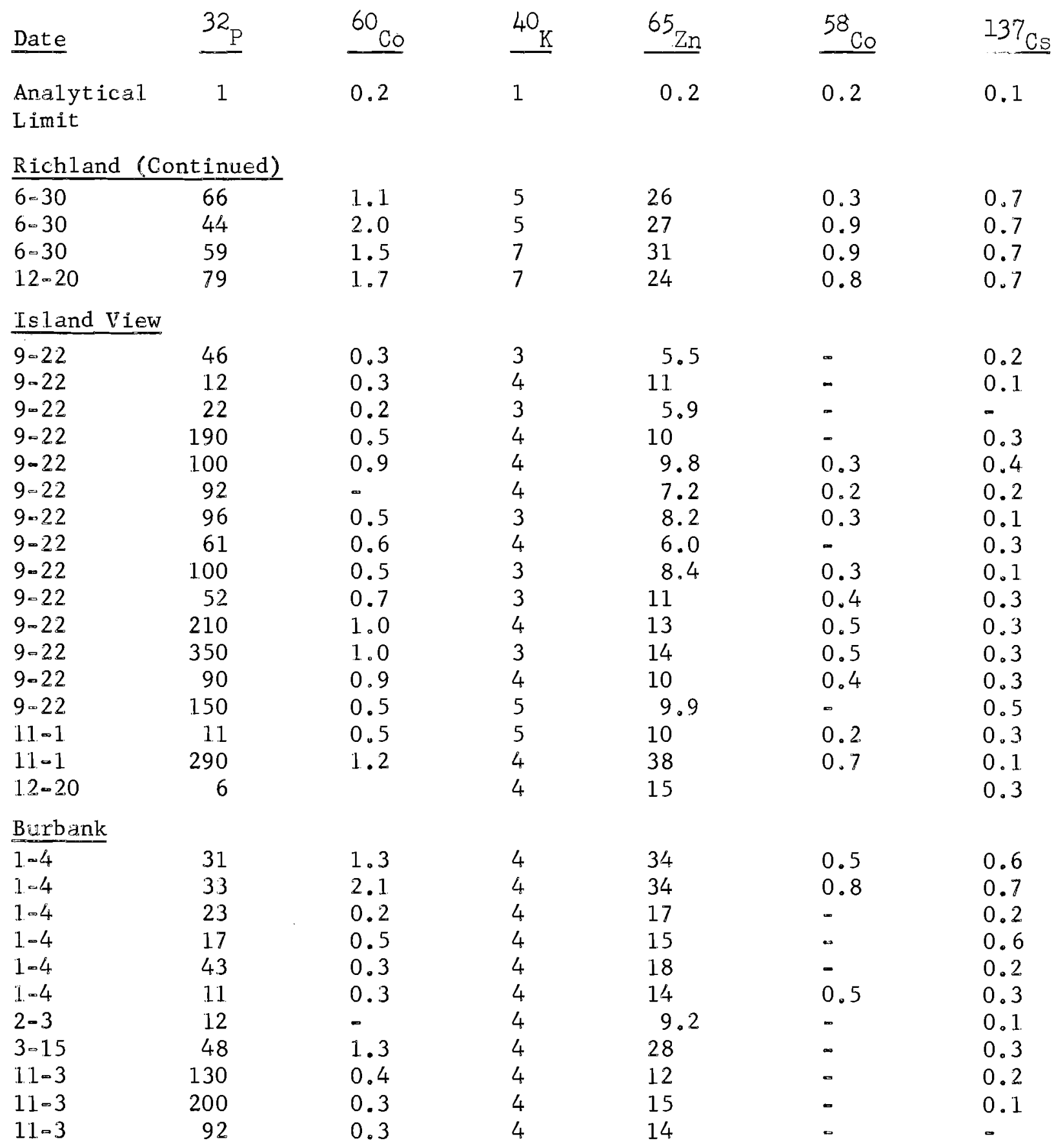

Results less than analytical limit are indicated by a $(-)$. No entry indicates no analysis made. 


\begin{tabular}{|c|c|c|c|c|c|c|}
\hline & & $\mathrm{TAB}$ & $\begin{array}{l}\text { DIX } \\
\text { onti }\end{array}$ & & & \\
\hline & & AT ION & DION & $S$ IN & & \\
\hline & & nits & curi & gram & & \\
\hline Date & $32_{\mathrm{P}}$ & ${ }^{60} \mathrm{Co}$ & $4 \mathrm{O}_{\mathrm{K}}$ & ${ }^{65} \mathrm{Zn}$ & ${ }^{58} \mathrm{Co}$ & ${ }^{137} \mathrm{C}$ \\
\hline $\begin{array}{l}\text { Analytical } \\
\text { Limit }\end{array}$ & 1 & 0.2 & 1 & 0.2 & 0.2 & 0.1 \\
\hline Burbank (Co & inue & & & & & \\
\hline $11-3$ & 6 & & 5 & 12 & - & 0.2 \\
\hline $11-3$ & 160 & & 7 & 21 & - & 0.2 \\
\hline $11-3$ & 88 & 1.0 & 6 & 18 & 0.7 & 0.3 \\
\hline $11-3$ & 40 & & 5 & 12 & - & 0.2 \\
\hline $12-13$ & 33 & & 3 & 8.2 & & - \\
\hline $12-13$ & 18 & & 3 & 8.3 & & 0.1 \\
\hline $12-13$ & 5 & & 4 & 10 & & 0.1 \\
\hline $12-13$ & 18 & & 3 & 14 & & - \\
\hline $12-13$ & 26 & 0.9 & 3 & 29 & - & 0.5 \\
\hline $12-13$ & 4 & 1.1 & 1 & 12 & - & 0.5 \\
\hline $12-13$ & 31 & & 3 & 20 & & - \\
\hline $12-13$ & 12 & & 3 & 11 & & - \\
\hline $12-13$ & 38 & & 3 & 20 & & - \\
\hline $12-13$ & 40 & & 5 & 22 & & - \\
\hline $12-13$ & 18 & & 4 & 9.8 & & 0.1 \\
\hline $12-13$ & 6 & & 3 & 11 & & 0.4 \\
\hline
\end{tabular}

McNary Dam

$10-18$

110

0.3

3

10

0.2

Results less than analytical 1 imit are indicated by a $(-)$. No entry indicates no analysis made. 


\section{APPENDIX $C$}

TABLE 2

CONCENTRATIONS OF RADIOCUCLIDES IN MUSCLE OF

BASS TAKEN FROM THE COLUMBIA RIVER - 1966

Units of picocuries per gram

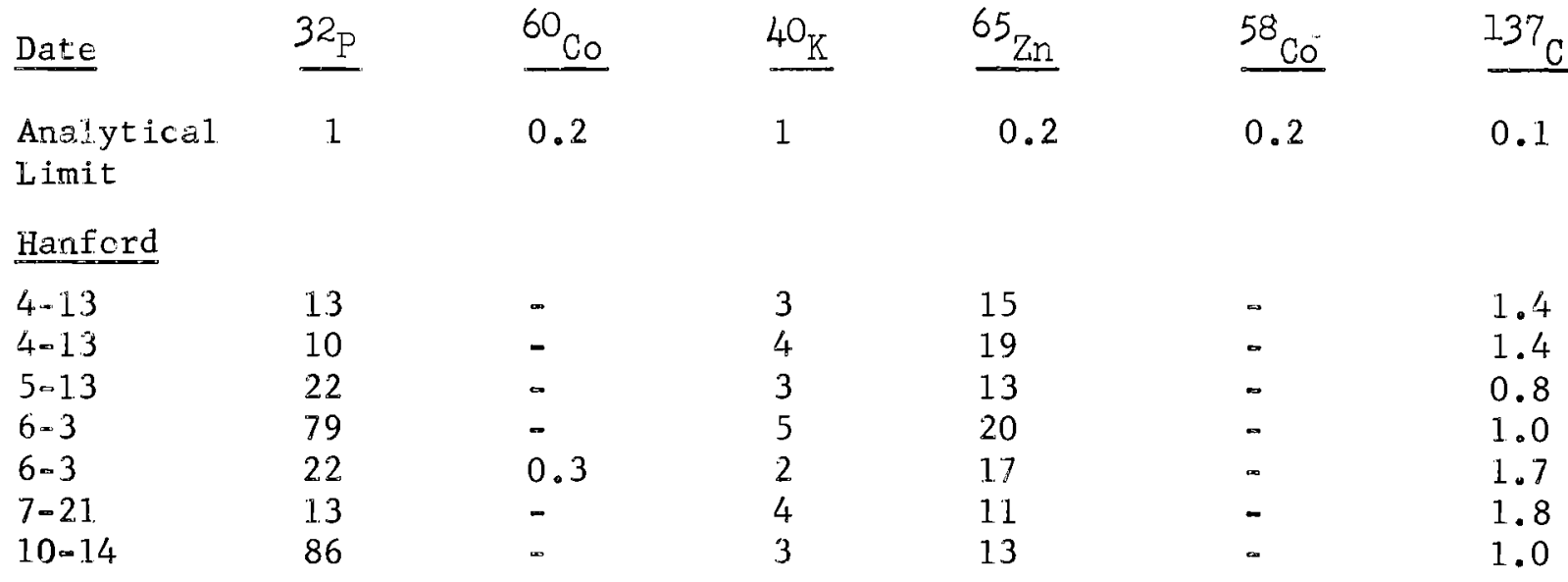

Ringold

$3-25$
$3-25$
$3-25$
$3-25$
$3-25$
$3-25$
$3-25$
$3-25$
$4-27$
$4-27$
$4-27$

$4-0.2$

-

$-$

Richland

$$
1 \mathrm{i}-2
$$

$1.1-2$

$\begin{array}{lll}93 & - & 5 \\ 26 & - & 4\end{array}$

$$
4
$$$$
2.4
$$

15

7.9
1.5

1.1

1.0

0.9

0.3

0.4

Island View

$11 \propto 1$.

17

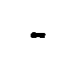

$\begin{array}{ll}- & 4 \\ - & 5 \\ - & 4 \\ - & 3\end{array}$

9.0

0.2

3- 15

$3-15$

$8=10$

$12-13$$$
1
$$

$\begin{array}{rr}4 & 9 \\ 5 & 13\end{array}$
9.7

9.0
0.3

0.7

Results less than analytical limit are indicated by a $(\infty)$. No entry indicates no analysis made. 
APPENDIX C

TABLE 3

\section{CONCENTRATIONS OF RADIONUCLIDES IN MUSCLE OF \\ CATFISH TAKEN FROM THE COLUMBIA RIVER - 1966}

$$
\text { Units of picocuries per gram }
$$

\begin{tabular}{|c|c|c|c|c|c|c|}
\hline Date & $32_{P}$ & $60 \mathrm{Co}$ & $4 \mathrm{O}_{\mathrm{K}}$ & ${ }^{65} \mathrm{Zn}$ & ${ }_{\mathrm{Co}}^{58}$ & $137 \mathrm{Cs}$ \\
\hline $\begin{array}{l}\text { Analytical } \\
\text { Limit }\end{array}$ & 1 & 0.2 & 1 & 0.2 & 0.2 & 0.1 \\
\hline \multicolumn{7}{|l|}{ Hanford } \\
\hline $\begin{array}{l}5-13 \\
5-13 \\
8-24\end{array}$ & $\begin{array}{r}310 \\
\text { LOST } \\
8\end{array}$ & 0.3 & $\begin{array}{l}3 \\
2 \\
2\end{array}$ & $\begin{array}{l}10 \\
- \\
5.4\end{array}$ & $\infty$ & $\begin{array}{l}0.3 \\
- \\
0.4\end{array}$ \\
\hline
\end{tabular}

Richland

$11-2$

25

$-5$

6.9

0.3

Island View

$\begin{array}{lr}7-6 & 3 \\ 7-6 & 7 \\ 7-6 & - \\ 7-6 & 5 \\ 7-6 & 4 \\ 9-22 & 5 \\ 9-22 & - \\ 9-22 & - \\ 9-22 & 4 \\ 9-22 & 2 \\ 11-1 & 66 \\ 11-1 & 1 \\ 11-1 & - \\ 12-20 & 1\end{array}$

$\begin{array}{ccc} & 6 & 2.8 \\ & 3 & 2.0 \\ 3 & 0.9 \\ & 3 & 1.9 \\ 3 & 2.1 \\ 4 & 4.1 \\ 4 & 1.5 \\ & 4 & 2.3 \\ & 4 & 4.3 \\ & 4 & 3.6 \\ & - & 1.6 \\ 3 & 1.5 \\ 3 & 1.9 \\ 4 & 1.4\end{array}$

Burbank

$1=4$

$1=4$

$2-3$

$2-3$

$3-15$

$3-15$

$3-15$

3-15

$3-15$

3-15

3-15

$3=15$

$\begin{array}{ll}- & 3 \\ - & 3 \\ - & 3 \\ - & 4 \\ - & 3 \\ - & 4 \\ 0.2 & 3 \\ - & 3 \\ - & 3 \\ - & 3 \\ - & 4\end{array}$

7.7
4.4
5.3
7.9
15
15
17
11
11
14
12
11

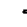

0.3

1.9

2.1

1.5

2.3

4.3

3.6

1.6

1.5

1.4

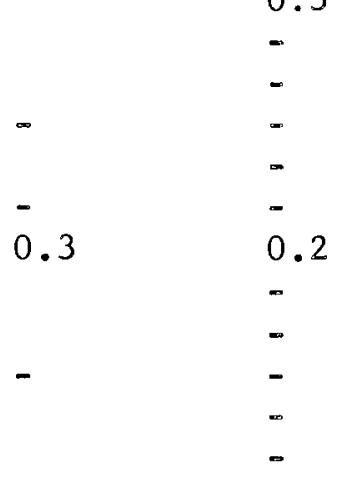

Results less than analytical limit are indicated by a $(\infty)$. No entry indicates no analys is made. 


\section{APPENDIX C \\ TABLE 3 (Continued)}

CONCENTRATIONS OF RADIONUCLIDES IN MUSCLE OF

CATFISH TAKEN FROM THE COLUMBIA RIVER - 1966

Date
Analytical

Units of picocuries per gram

\begin{tabular}{|c|c|c|c|c|c|c|}
\hline $3-51$ & 2 & - & 3 & 13 & - & - \\
\hline $3-15$ & 2 & - & 3 & 3.1 & - & - \\
\hline $4=20$ & 2 & & 3 & 7.3 & - & 0.1 \\
\hline $4-20$ & 2 & & 2 & 12 & - & 0.4 \\
\hline $4-20$ & 3 & & 3 & 8.7 & $\infty$ & - \\
\hline $4-20$ & 3 & & 2 & 16 & - & - \\
\hline $4-20$ & 3 & & 3 & 9.8 & - & - \\
\hline $4-20$ & 2 & & 3 & 14 & - & 0.1 \\
\hline $4-20$ & 3 & & 2 & 12 & - & 0.1 \\
\hline $4-20$ & - & & 3 & 9.0 & - & 0.1 \\
\hline $4 \cdot 20$ & - & & 3 & 7.6 & - & 0.1 \\
\hline $4-20$ & - & - & 3 & 6.7 & - & 0.2 \\
\hline $5-6$ & - & & 2 & 1.3 & $=$ & 0.1 \\
\hline $5-6$ & - & & 3 & 8.6 & - & 0.1 \\
\hline $5-20$ & 8 & & 4 & 13 & - & 0.2 \\
\hline $5-20$ & 12 & & 4 & 12 & - & 0.1 \\
\hline $5-20$ & $=$ & & 3 & 11 & - & - \\
\hline $5-20$ & 12 & & 2 & 9.2 & - & 0.1 \\
\hline $5-20$ & 2 & & 3 & 2.2 & - & 0.1 \\
\hline $5-20$ & 9 & & 3 & 8.4 & $=$ & 0.1 \\
\hline $5-20$ & 6 & & 3 & 12 & - & - \\
\hline $5-20$ & 6 & & 3 & 0.4 & $\infty$ & $\approx$ \\
\hline $6-9$ & 3 & - & 3 & 6.6 & - & 0.1 \\
\hline - & 3 & - & 3 & 9.1 & $\infty$ & 0.3 \\
\hline - & 3 & - & 3 & 11 & - & 0.2 \\
\hline - & 4 & $=$ & 2 & 8.7 & $=$ & 0.2 \\
\hline$\infty$ & 3 & - & 3 & 12 & - & 0.1 \\
\hline - & 4 & - & 3 & 14 & $=$ & 0.1 \\
\hline$=$ & 4 & $=$ & 3 & 13 & - & - \\
\hline - & 3 & $=$ & 3 & 8.9 & - & 0.1 \\
\hline$=$ & 4 & - & 3 & 13 & - & - \\
\hline$=$ & 5 & - & 3 & 15 & - & - \\
\hline $8-2$ & 8 & & 3 & 10 & - & 0.1 \\
\hline $8-2$ & 9 & & 3 & 8.6 & - & - \\
\hline $8-2$ & 7 & & 3 & 8.5 & $\infty$ & - \\
\hline $8-2$ & 2 & & 3 & 5.4 & - & - \\
\hline $8-2$ & 3 & & 3 & 10 & - & 0.2 \\
\hline
\end{tabular}

Results less than analytical limit are indicated by a $(-)$. No entry indicates no analysis made. 
APPENDIX C

CONCENTRATIONS OF RADIONUCLIDES IN MUSCLE OF CATFISH TAKEN FROM THE COLUMBIA RIVER - 1966 Units of picocuries per gram

\begin{tabular}{|c|c|c|c|c|c|c|}
\hline Date & $32_{P}$ & ${ }^{60} \mathrm{Co}$ & $4 \mathrm{O}_{\mathrm{K}}$ & ${ }^{65} \mathrm{Zn}$ & ${ }^{58} \mathrm{Co}$ & ${ }^{137} \mathrm{Cs}$ \\
\hline $\begin{array}{l}\text { Analytical } \\
\text { Limit }\end{array}$ & 1 & 0.2 & 1 & 0.2 & 0.2 & 0.1 \\
\hline \multicolumn{7}{|c|}{ Burbank (Cont inued) } \\
\hline $8-10$ & 4 & & 3 & 4.2 & $\infty$ & - \\
\hline $8-10$ & 1 & 0.2 & 3 & 8.6 & - & 0.2 \\
\hline $8-10$ & 4 & & 3 & 6.5 & - & 0.2 \\
\hline $8-10$ & 4 & & 4 & 10 & $-\infty$ & 0.2 \\
\hline $8 \cdot 16$ & 3 & - & 3 & 8.3 & - & 0.1 \\
\hline $8-16$ & 7 & & 3 & 6.9 & - & - \\
\hline $8=16$ & 3 & & 3 & 9.2 & $=$ & - \\
\hline $8=16$ & 8 & & 4 & 14 & - & 0.3 \\
\hline $8-16$ & - & & 3 & 10 & $\infty$ & 0.2 \\
\hline $8-30$ & 1 & & 3 & 6.0 & & 0.1 \\
\hline $8-30$ & 4 & & 4 & 7.8 & & 0.1 \\
\hline $8-30$ & 4 & & 3 & 7.4 & & $=$ \\
\hline $8-30$ & 3 & & 3 & 6.7 & & 0.1 \\
\hline $9-15$ & 44 & & 3 & 6.6 & - & 0.2 \\
\hline $10-10$ & 41 & & 3 & 10 & - & - \\
\hline $10-10$ & 14 & & 3 & 6.0 & - & 0.1 \\
\hline $10-10$ & 95 & - & 3 & 12 & - & - \\
\hline $10-10$ & 21 & 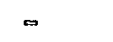 & 2 & 6.2 & - & 0.1 \\
\hline $10-10$ & 45 & & 3 & 8.1 & - & 0.1 \\
\hline $11-3$ & 5 & & 4 & 6.0 & - & 0.1 \\
\hline $12-13$ & 12 & & 18 & 49 & & - \\
\hline
\end{tabular}

Results less than analytical limit are indicated by a $(-)$. No entry indicates no analysis made. 


\section{APPENDIX C}

TABLE 4

CONCENTRATIONS OF RADIONUCLIDES IN MUSCLE OF

CRAPPIE TAKEN FROM THE COLUMBIA RIVER - 1966

Units of picocuries per gram

\begin{tabular}{|c|c|c|c|c|c|c|}
\hline Date & $32_{\mathrm{P}}$ & $\frac{60 \mathrm{Co}}{}$ & $40_{K}$ & $65 \mathrm{Zn}$ & ${ }^{58} \mathrm{C}$ & ${ }^{137} \mathrm{Cs}$ \\
\hline $\begin{array}{l}\text { Analytical } \\
\text { Limit }\end{array}$ & 1 & 0.2 & 1 & 0.2 & 0.2 & 0.1 \\
\hline Island View & & & & & & \\
\hline $\begin{array}{l}9-22 \\
9-22\end{array}$ & $\begin{array}{l}54 \\
86\end{array}$ & - & $\begin{array}{l}4 \\
3\end{array}$ & $\begin{array}{l}3.0 \\
9.4\end{array}$ & - & - \\
\hline
\end{tabular}

Burbank

\begin{tabular}{|c|c|c|c|c|c|c|}
\hline $1-4$ & 13 & 0.2 & 5 & 17 & & 0.1 \\
\hline $2-3$ & 2 & - & 3 & 15 & - & 0.1 \\
\hline $3-15$ & - & - & 3 & 14 & - & 0.1 \\
\hline $3-15$ & - & - & 4 & 12 & - & - \\
\hline $4-20$ & 3 & - & 4 & 21 & - & 0.1 \\
\hline- & 1 & - & 4 & 14 & - & - \\
\hline - & 1 & - & 3 & 13 & - & 0.2 \\
\hline . & 1 & - & 3 & 13 & - & - \\
\hline- & 3 & - & 3 & 11 & - & 0.1 \\
\hline$=$ & 2 & - & 3 & 13 & $=$ & 0.2 \\
\hline - & 1 & - & 3 & 12 & - & 0.2 \\
\hline $5-6$ & 2 & & 2 & 89 & - & 0.3 \\
\hline $8-2$ & 31 & & 4 & 0.4 & & 0.2 \\
\hline $8-2$ & 11 & & 5 & 9.1 & & 0.1 \\
\hline $8-2$ & 10 & & 4 & 15 & & - \\
\hline $8-10$ & 14 & & 3 & 16 & - & 0.2 \\
\hline $8-16$ & 9 & & 6 & 11 & - & 0.3 \\
\hline $8-16$ & 6 & & 4 & 9.6 & - & - \\
\hline $9-15$ & 38 & & 2 & 10 & 0.2 & - \\
\hline $10-10$ & 12 & & 3 & 7.1 & $=$ & 0.1 \\
\hline $10-10$ & 6 & & 5 & 5.4 & 0.2 & 0.1 \\
\hline $10-10$ & 190 & & 2 & 10 & - & 0.1 \\
\hline $11-13$ & 110 & & 7 & 19 & - & 0.1 \\
\hline $12-13$ & 9 & & & 10 & & - \\
\hline
\end{tabular}

Results less than analytical limit are indicated by a $(-)$. No entry indicates no analys is made. 
APPENDIX C

TABLE 5

CONCENTRATIONS OF RADIONJCLIDES IN MUSCLE OF

PERCH TAKEN FROM THE COLUMBIA RIVER - 1966 Units of picocuries per gram

\begin{tabular}{|c|c|c|c|c|c|}
\hline Date & $32_{\mathrm{P}}$ & ${ }^{60} \mathrm{Co}$ & $4 \mathrm{O}_{\mathrm{K}}$ & ${ }^{65} \mathrm{Zn}$ & ${ }^{58} \mathrm{Co}$ \\
\hline $\begin{array}{l}\text { Analytical } \\
\text { Limit }\end{array}$ & 1 & 0.2 & 1 & 0.2 & 0.2 \\
\hline
\end{tabular}

Hanford

$\begin{array}{rrrrrrl}4-13 & 6 & - & 8 & 0.7 & - & - \\ 5-13 & \text { LOST } & & 2 & 0.2 & & - \\ 8-24 & 15 & - & 5 & 17 & - & 0.9 \\ 8-24 & 39 & - & 5 & 10 & - & - \\ 8-24 & 39 & - & 4 & 10 & - & 0.6 \\ 9-10 & 48 & - & 5 & 19 & - & 0.9 \\ 12-6 & 100 & - & 5 & 11 & - & 0.6\end{array}$

Richland

\section{$11-2$}

$12-20$

320

16

Island View

$7-6$

9

Burbank

\section{$1-4$}

1-4

1-4

2-3

2-3

3-15

3-15

3-15

3-15

3-15

3-15

3-15

3-15

4- 20

4-20

4-20

4-20

5-6

5-6

5-6

$\begin{array}{rll}5 & & 4 \\ 8 & & 4 \\ 26 & - & - \\ 6 & - & 3 \\ 7 & - & 5 \\ 3 & - & 6 \\ - & - & 6 \\ 3 & - & 3 \\ 5 & - & 3 \\ 2 & - & 5 \\ 11 & - & 3 \\ - & - & 3 \\ 4 & - & 4 \\ 1 & - & 4 \\ 2 & - & 3 \\ - & - & 3 \\ 1 & & 3 \\ 5 & & 5 \\ 5 & & 2 \\ 5 & & \end{array}$

5

3.5

$\begin{array}{ll}4 & 14 \\ 4 & 14\end{array}$

3

5

6

4
28

21

1.1

1.1

Results less than analytical limit are indicated by a $(-)$. No entry indicates no analys is made. 
APPENDIX $C$

TABLE 5 (Continued)

\section{CONCENTRATIONS OF RADIONUCLIDES IN MUSCLE OF}

PERCH TAKEN FROM THE COLUMBIA RIVER - 1966

$$
\text { Lnits of picocuries per gram }
$$

\begin{tabular}{|c|c|c|c|c|c|c|}
\hline Date & $32_{P}$ & ${ }^{60} \mathrm{Co}$ & $4 \mathrm{O}_{\mathrm{K}}$ & ${ }^{65} \mathrm{Zn}$ & ${ }^{58} \mathrm{Co}$ & $137 \mathrm{Cs}$ \\
\hline $\begin{array}{l}\text { Analytical } \\
\text { Limit }\end{array}$ & 1 & 0.2 & 1 & 0.2 & 0.2 & 0.1 \\
\hline
\end{tabular}

Biurbank (Continued)

\begin{tabular}{|c|c|c|c|c|c|c|}
\hline $5-6$ & - & & 3 & 8.5 & - & 0.4 \\
\hline $5-6$ & 4 & & 4 & 8.7 & - & - \\
\hline $5-6$ & 72 & & 4. & 9.8 & - & 0.2 \\
\hline $5-6$ & 1 & & 3 & 7.8 & - & - \\
\hline $5-6$ & - & & 3 & 13 & - & 0.3 \\
\hline $5-20$ & 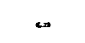 & & 2 & 12 & - & 0.2 \\
\hline $5-20$ & 4 & & 2 & 14 & - & 0.1 \\
\hline $5-20$ & 2 & & 3 & 15 & - & - \\
\hline $5-20$ & 6 & & 3 & 17 & - & 0.1 \\
\hline $5-20$ & - & & 4 & 14 & - & - \\
\hline $6-9$ & 9 & - & 3 & 15 & - & 0.1 \\
\hline $6-9$ & 4 & $\infty$ & 3 & 11 & - & - \\
\hline $6-9$ & 7 & - & 5 & 13 & - & 0.1 \\
\hline $6-9$ & 2 & - & 1 & 8.0 & - & $=$ \\
\hline $6-9$ & . & - & 3 & 14 & - & 0.5 \\
\hline 6.9 & 12 & 0.2 & 3 & 12 & - & 0.1 \\
\hline $6-9$ & 3 & 0.2 & 3 & 11 & - & $\infty$ \\
\hline $7 \cdot .27$ & 20 & - & 3 & 11 & - & 0.2 \\
\hline $7-28$ & 11 & & 5 & 8.9 & - & - \\
\hline $7-27$ & 40 & & 5 & 11 & & 0.2 \\
\hline $7-27$ & 6 & & 2 & 8.9 & - & 0.1 \\
\hline $7-27$ & 28 & & 5 & 13 & - & 0.1 \\
\hline $7-27$ & 14 & & - & 8.6 & . & 0.2 \\
\hline $7-27$ & 5 & & 3 & 7.4 & - & - \\
\hline $7-27$ & 17 & & 4 & 7.9 & - & - \\
\hline $7-27$ & 22 & & 3 & 8.6 & & - \\
\hline $7-27$ & 22 & & 4 & 13 & $\infty$ & 0.2 \\
\hline $7-27$ & 67 & & 4 & 9.2 & . & 0.1 \\
\hline $7-27$ & 3 & - & 3 & 10 & - & 0.1 \\
\hline $7-27$ & 14 & & 3 & 11 & - & 0.2 \\
\hline $7-27$ & 32 & & 2 & 12 & - & 0.1 \\
\hline $7-27$ & 21 & & 3 & 9.2 & - & - \\
\hline $8-2$ & 3 & & 4 & 1.3 & & - \\
\hline $8-2$ & 14 & & 6 & 9.9 & & 0.1 \\
\hline $8-2$ & 13 & & 3 & 7.5 & & - \\
\hline $8-2$ & 34 & & 2 & 13 & & 0.1 \\
\hline $8-2$ & 15 & & 4 & 11 & & \\
\hline
\end{tabular}

Results less than analytical limit are indicated by a $(-)$. No entry indicates no analys is made. 
APPENDIX C

TABLE 5 (Continued)

CONCENTRAT IONS OF RADIONUCLIDES IN MUSCLE OF

PERCH TAKEN FROM THE COLUMBIA RIVER - 1966

Units of picocuries per gram

\begin{tabular}{|c|c|c|c|c|c|c|}
\hline Date & $32_{\mathrm{P}}$ & ${ }^{60} \mathrm{CO}$ & $40_{K}$ & $65 \mathrm{zn}$ & ${ }^{58} \mathrm{Co}$ & $137 \mathrm{Cs}$ \\
\hline $\begin{array}{l}\text { Analytical } \\
\text { Limit }\end{array}$ & 1 & 0.2 & 1 & 0.2 & 0.2 & 0.1 \\
\hline \multicolumn{7}{|c|}{ Burbank (Continued) } \\
\hline $8-10$ & 7 & \multirow{23}{*}{0.2} & 3 & 8.5 & & $\infty$ \\
\hline $8-10$ & 9 & & 3 & 8.8 & - & - \\
\hline $8-30$ & 9 & & 3 & 7.2 & & - \\
\hline $8-30$ & 3 & & 5 & 7.9 & & - \\
\hline $9-15$ & 29 & & 4 & 9.5 & - & $\infty$ \\
\hline $9-15$ & 66 & & 3 & 8.8 & - & 0.1 \\
\hline $9-15$ & $=$ & & 6 & 13 & $=$ & - \\
\hline $10=10$ & 7 & & 6 & 7.2 & - & 0.4 \\
\hline $10=10$ & 33 & & 4 & 6.8 & - & - \\
\hline $10-10$ & 31 & & 4 & 9.0 & - & 0.2 \\
\hline $10-10$ & 80 & & 4 & 6.8 & & $\infty$ \\
\hline $10-10$ & 35 & & 4 & 6.9 & - & 0.2 \\
\hline $10-10$ & 34 & & 4 & 11 & - & 0.1 \\
\hline $10-10$ & 17 & & 6 & 7.6 & $\infty$ & - \\
\hline $11-3$ & 14 & & 5 & 9.4 & & - \\
\hline $11-3$ & 21 & & 3 & 10 & & 0.3 \\
\hline $12-13$ & - & & 3 & 12 & & 0.7 \\
\hline $12-13$ & 3 & & 3 & 12 & & - \\
\hline $12-13$ & 3 & & 6 & 7.1 & & - \\
\hline $12-13$ & 3 & & 4 & 12 & & - \\
\hline $12-13$ & 6 & & $=$ & 12 & & - \\
\hline $12-13$ & 5 & & - & 9.0 & & - \\
\hline $12-13$ & 9 & & 1 & 10 & & 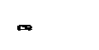 \\
\hline
\end{tabular}

Results less than analytical limit are indicated by a $(-)$. No entry indicates no analysis made. 
APPENDIX C

TABLE 6

\section{CONCENTRATIONS OF RADIONUCLIDES IN MUSCLE OF MISCELLANEOUS FISH TAKEN FROM THE COLUMBIA RIVER - 1966 \\ Units of picocuries per gram}

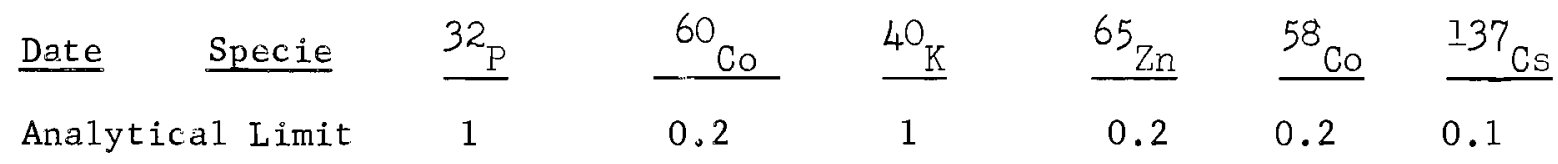

\section{Priest Rapids}

1-18 Trout

$5-2$

Sturgeon

$6-13$

$10=5$

$10-5$

Carp

Trout

Trout

Coyate Rapids

$\begin{array}{lll}1-13 & \text { Steelhead } & 3 \\ 1-14 & \text { Steelhead } & 3 \\ 3-1 & \text { Steelhead } & 5 \\ 3-4 & \text { Steelhead } & - \\ 3-4 & \text { Steelhead } & = \\ 9-28 & \text { Steelhead } & - \\ 9-28 & \text { Steelhead } & - \\ 9-28 & \text { Steelhead } & - \\ 9-28 & \text { Steelhead } & - \\ 10-14 & \text { Steelhead } & - \\ 10-14 & \text { Steelhead } & -\end{array}$

Hanford

$\begin{array}{llr}\text { 4-13 } & \text { Carp } & 120 \\ 4-13 & \text { Sturgeon } & 16 \\ 4-13 & \text { Sturgeon } & 7 \\ 4-13 & \text { Sturgeon } & 37 \\ 4-13 & \text { Sturgeon } & 7 \\ 5-13 & \text { Sturgeon } & 17 \\ 5-13 & \text { Sturgeon } & 31 \\ 5-13 & \text { Sturgeon } & 19 \\ 6-3 & \text { Sturgeon } & 18 \\ 6-3 & \text { Sturgeon } & 20 \\ 6-3 & \text { Sturgeon } & 10 \\ 7-21 & \text { Steelhead } & 0 \\ 8-24 & \text { Carp } & 15 \\ 8-24 & \text { Carp } & 5 \\ 8-24 & \text { Sturgeon } & 14\end{array}$

-

3
3
3
4
4

1.3

$$
5.6
$$$$
26
$$

1.3

1.1

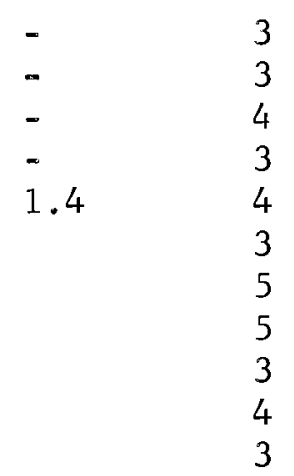

$\begin{array}{ll}- & 4 \\ - & 3 \\ - & 3 \\ 0.3 & 4 \\ 0.2 & 3 \\ - & 2 \\ - & 3 \\ 0.3 & 2 \\ 0.4 & 3 \\ - & 4 \\ - & 3 \\ - & 3 \\ - & 4 \\ - & 5 \\ 0.2 & 5\end{array}$

$\begin{array}{cll}- & - & - \\ - & - & - \\ 0.3 & - & - \\ 0.9 & - & - \\ 11 & 0.6 & 0.2 \\ & & 0.1 \\ & & - \\ & & - \\ & & - \\ - & & -\end{array}$

$\begin{array}{cll}33 & - & - \\ 9.2 & 0.2 & 0.6 \\ 20 & 0.3 & 0.7 \\ 9.2 & 0.2 & 0.6 \\ 15 & 0.4 & 0.5 \\ 12 & 0.2 & 0.5 \\ 16 & 0.2 & 0.7 \\ 16 & - & 0.8 \\ 13 & 0.2 & 1.2 \\ 14 & - & 0.7 \\ 14 & 0.2 & 0.8 \\ - & - & - \\ 37 & - & 0.2 \\ 4.1 & - & - \\ 11 & - & 0.7\end{array}$

Results less than analytical limit are indicated by a $(-)$. No entry indicates no analysis made. 
APPENDIX $C$

TABLE 6 (Cont inued)

CONCENTRATIONS OF RADIONUCLIDES IN MUSCLE OF MISCELLANEOUS FISH TAKEN FROM THE COLUMBIA RIVER - 1966 Units of picocuries per gram

\begin{tabular}{|c|c|c|c|c|c|c|c|}
\hline Date & Specie & $32_{p}$ & ${ }^{60}$ & $40_{K}$ & $65_{\mathrm{Zn}}$ & ${ }^{58} \mathrm{Co}$ & $137 \mathrm{Cs}$ \\
\hline \multicolumn{2}{|c|}{ Analytical Limit } & 1 & 0.2 & 1 & 0.2 & 0.2 & 0.1 \\
\hline \multicolumn{8}{|c|}{ Ringold } \\
\hline$\overline{1-12}$ & Trout & 96 & 0.6 & 5 & 64 & - & - \\
\hline $1-12$ & Trout & 130 & 1.2 & 7 & 92 & 0.3 & - \\
\hline $1-12$ & Trout & 130 & 0.6 & 4 & 90 & - & - \\
\hline $1-27$ & Trout & 69 & 0.9 & 5 & 47 & 0.3 & 0.2 \\
\hline $2-16$ & Trout & 76 & 0.9 & 6 & 73 & $=$ & - \\
\hline $2-16$ & Trout & 86 & - & 4 & 57 & - & - \\
\hline $2-16$ & Trout & 120 & - & 5 & 54 & - & 0.2 \\
\hline $3-18$ & Trout & 110 & 0.9 & 5 & 69 & - & 0.3 \\
\hline $3-22$ & Trout & 150 & 0.4 & 11 & 41 & - & 0.3 \\
\hline $10-3$ & Trout & 120 & 0.3 & 5 & 21 & - & 0.2 \\
\hline $10-3$ & Trout & 270 & 0.4 & 5 & 28 & - & 0.3 \\
\hline $12-8$ & Trout & 250 & 0.3 & 6 & 35 & - & 0.1 \\
\hline $12-8$ & Trout & 350 & 0.2 & 5 & 42 & - & - \\
\hline
\end{tabular}

Richland

\begin{tabular}{llrlllll}
\hline $4-22$ & Trout & 84 & - & 4 & 4.9 & - & 0.1 \\
$11-2$ & Carp & 320 & 0.2 & 4 & 59 & - & - \\
$11-2$ & Carp & 170 & 0.2 & 7 & 82 & - & - \\
$11-2$ & Carp & 300 & - & 7 & 58 & - & - \\
$11-2$ & Carp & 450 & - & 6 & 60 & - & - \\
$11-2$ & Carp & 400 & - & 5 & 64 & - & - \\
$12-20$ & Carp & 21 & 0.4 & 4 & 37 & - & 0.7
\end{tabular}

Is 1 and View

\begin{tabular}{|c|c|c|c|c|c|c|c|}
\hline $7-6$ & Carp & 12 & & 3 & 9.2 & - & - \\
\hline $7-6$ & Carp & 2 & & 4 & 10 & - & 0.1 \\
\hline $7-6$ & Carp & 3 & & 5 & 66 & - & - \\
\hline $7-6$ & Carp & 4 & & 4 & 35 & - & 0.2 \\
\hline $9-22$ & Bluegill & 3 & & 4 & 1.2 & - & - \\
\hline $9-22$ & Carp & 32 & & 4 & 5.8 & - & $\infty$ \\
\hline $9-22$ & Carp & 26 & & 5 & 7.9 & - & - \\
\hline $9-22$ & Carp & 2 & - & 3 & 4.1 & - & - \\
\hline $9-22$ & Carp & - & & 3 & 1.1 & - & - \\
\hline $9-22$ & Carp & - & & 4 & 2.4 & - & - \\
\hline $9-22$ & Sturgeon & 3 & & 4 & 3.4 & - & - \\
\hline $11-1$ & Carp & 2 & & 2 & 3.8 & - & 0.1 \\
\hline $11-1$ & Carp & - & & 3 & 2.6 & - & - \\
\hline
\end{tabular}

Results less than analytical limit are indicated by a $(-)$. No entry indicates no analysis made. 


\section{APPENDIX C}

TABLE 6 (Continued)

\section{CONCENIRATIONS OF RADIONUCLIDES IN MUSCLE OF MISCELLANEOUS FISH TAKEN FROM THE COLUMBIA RIVER - 1966} Units of picocuries per gram

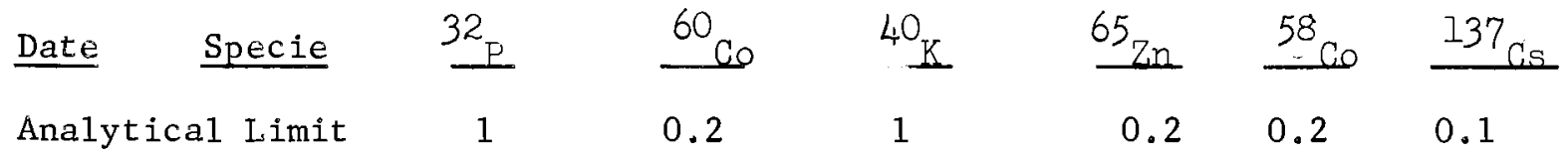

Is land View (Cont inued)

\begin{tabular}{llr}
\hline $11-1$ & Carp & 2 \\
$11-1$ & Carp & 44 \\
$12-20$ & Carp & - \\
$12-20$ & Carp & 32 \\
$12-20$ & Carp & 2 \\
$12-20$ & Carp & 38
\end{tabular}

$\begin{array}{ll} & 3 \\ & 3 \\ 0.8 \quad 3 \\ & 3 \\ & 3 \\ & 2\end{array}$

Burbank

$1=4$

$2-3$

$3-15$

$3=15$

$3-15$

4-20

Sucker

Carp

Carp

Carp

4- 20

$4=20$

Carp

Carp

Carp

$4=20$

Carp

4-20

Carp

44

32

2
38

3
3
3
3
3
2

$0.2 \quad 0.2$

$4-20$

Carp

$5-20$

Carp

$\begin{array}{ll}72 & 0.5 \\ - & - \\ 1 & - \\ 1 & -\end{array}$

3

$$
\begin{aligned}
& 5.8 \\
& 9.8 \\
& 1.1 \\
& 68 \\
& 6.4 \\
& 72
\end{aligned}
$$

5- 20

$5-20$

$5-20$

$5-20$

6-9

6-9

6.9

$6-9$

$6-9$

$6-9$

6-9

$6-9$

$6-9$

6.9

6.9

6-9

Sturgeon

Carp

Carp

Carp

Carp

Carp

Carp

Carp

Carp

Carp

Carp

Carp

Carp

Carp

Sturgeon

Sturgeon

B1uegil1.

Bluegil1

9
4
46
21
4
5
9
3
62
22
33
51
31
17
28
41
12
22
8.7
24
39
23
11

Results less than analytical limit indicated by a $(-)$. No entry indicates no analysis made. 
APPENDIX C

TABLE 6 (Continued)

CONCENTRATIONS OF RADIONUCLIDES IN MUSCLE OF

MISCELLANEOUS FISH TAKEN FROM THE COLUMBIA RIVER - 1966

$$
\text { Units of picocuries per gram }
$$

\begin{tabular}{|c|c|c|c|c|c|c|c|}
\hline Date & Specie & $32_{P}$ & ${ }^{60} \mathrm{Co}$ & $4 \mathrm{O}_{\mathrm{K}}$ & ${ }^{65} \mathrm{Zn}$ & ${ }^{58} \mathrm{Co}$ & ${ }^{137} \mathrm{Cs}$ \\
\hline Analytica & a1 Limit & 1 & 0.2 & 1 & 0.2 & 0.2 & 0.1 \\
\hline$\frac{\text { Burbank }}{7.27}$ & Sturgeon & 8 & & 3 & 7.7 & - & 0.2 \\
\hline $7=27$ & Sturgeon & 3 & & 3 & 5.3 & - & - \\
\hline $7-27$ & Sturgeon & 2 & & 3 & 11 & - & 0.1 \\
\hline $7-27$ & Sturgeon & 14 & & 3 & 6.9 & - & - \\
\hline $7-27$ & Sturgeon & 37 & & 2 & 15 & $=$ & - \\
\hline $7-27$ & Shad & 9 & & 4 & 2.6 & - & - \\
\hline $7-27$ & Shad & 3 & & 5 & 4.4 & - & 0.1 \\
\hline $7-27$ & Shad & 16 & & 4 & 3.0 & - & - \\
\hline $8-2$ & Sturgeon & 15 & & 2 & 11 & - & 0.1 \\
\hline $8-2$ & Carp & 19 & & 4 & 33 & - & - \\
\hline $8=2$ & Shad & 25 & & 3 & 38 & - & - \\
\hline $8-2$ & Shad & 17 & & 3 & 15 & - & - \\
\hline $8-2$ & Shad & 6 & & 4 & 12 & & 0.2 \\
\hline $8-2$ & Carp & 7 & & 2 & 2.1 & . & - \\
\hline $8-2$ & Carp & 1 & & 4 & 1.0 & & $=$ \\
\hline $8-10$ & Carp & 8 & - & 3 & 31 & - & - \\
\hline $8-10$ & Carp & 14 & - & 3 & 20 & - & 0.2 \\
\hline $8-10$ & Garp & 16 & & 4 & 22 & - & - \\
\hline $8-10$ & Carp & 9 & - & 3 & 15 & - & - \\
\hline $8=10$ & Carp & 7 & & 4 & 24 & $=$ & - \\
\hline $8 \cdot 16$ & Carp & 23 & 0.2 & 3 & 34 & - & 0.3 \\
\hline $8-16$ & Carp & 5 & & 3 & 46 & - & - \\
\hline $8-16$ & Carp & 13 & - & 3 & 10 & - & 0.1 \\
\hline $8-16$ & Carp & LOST & & 3 & 17 & - & - \\
\hline $8-16$ & Carp & 9 & & 3 & 38 & - & $=$ \\
\hline $8-30$ & Carp & 4 & & 3 & 29 & & - \\
\hline $8=30$ & Carp & 5 & & 3 & 29 & & $=$ \\
\hline $8-30$ & Carp & 12 & & 3 & 20 & & - \\
\hline $8-30$ & Carp & 3 & & 4 & 28 & & - \\
\hline $8-30$ & Carp & 6 & & 4 & 23 & & - \\
\hline $8-30$ & Sturgeon & 7 & & 4 & 7.9 & & 0.1 \\
\hline $9-1.5$ & Steelhead & - & & 3 & - & - & 0.1 \\
\hline $9-15$ & Carp & 43 & & 4 & 25 & - & - \\
\hline $9-15$ & Carp & 42 & & 3 & 18 & - & - \\
\hline $9-15$ & Shad & 130 & & 4 & 5.2 & - & - \\
\hline $10-10$ & Carp & 24 & - & 3 & 26 & $=$ & - \\
\hline $10-10$ & Carp & 33 & & 4 & 10 & - & \\
\hline
\end{tabular}

Results less than analytical limit indicated by a ( ) . No entry indicates no analys is made. 


$$
\begin{gathered}
\text { APPENDIX C } \\
\text { TABLE } 6 \text { (Continued) }
\end{gathered}
$$

CONCENTRATTONS OF RADIONUCLIDES IN MUSCLE OF MISCELIANEOUS FISH TAKEN FROM THE COLUMBIA RIVER - 1966

\begin{tabular}{|c|c|c|c|c|c|c|c|}
\hline Date & Specie & $32_{\mathrm{P}}$ & مC & $40_{K}$ & $65 \mathrm{7n}$ & مC & $137 \mathrm{C}$ \\
\hline Analyt & al Limit & 1 & 0.2 & 1 & 0.2 & 0.2 & 0.1 \\
\hline Burban & (Continued & & & & & & \\
\hline$\overline{11-3}$ & Carp & 55 & & 5 & 22 & - & 0.1 \\
\hline $11-3$ & Garp & 88 & & 5 & 36 & - & 0.2 \\
\hline $12-13$ & Carp & 5 & & 4 & 8.2 & & - \\
\hline $12-13$ & Carp & - & & 3 & 21 & & - \\
\hline $12-13$ & Carp & - & & 3 & 20 & & - \\
\hline $12-13$ & Carp & 21. & & 4 & 19 & & 0.2 \\
\hline $12=13$ & Caxp & 4 & & 4 & 20 & & - \\
\hline MeNary & & & & & & & \\
\hline $10-1.8$ & Sturgeon & 7 & & 4 & 4.8 & - & 0.1 \\
\hline $10 \cdot 18$ & Sturgeon & 18 & & 3 & 4.1 & - & 0.1 \\
\hline $10-1.8$ & Sturgeon & 10 & & 4 & 4.0 & - & 0.2 \\
\hline $10-18$ & Sturgeon. & 14 & & 3 & 3.0 & - & 0.2 \\
\hline $10-18$ & Sturgeon & 8 & & 3 & 4.6 & - & 0.2 \\
\hline $10-18$ & Ste€lhead & - & & 3 & - & - & $\infty$ \\
\hline $11-7$ & Sturgeor. & 3 & & 4 & 4.4 & & 0.1 \\
\hline $11-17$ & Sturgeon & 38 & & 4 & 5.9 & & 0.1 \\
\hline $11-17$ & Sturgeon & 52 & & 3 & 8.7 & & 0.1 \\
\hline $11-17$ & Sturgeon & 18 & & 2 & 8.1 & & 0.1 \\
\hline
\end{tabular}

Units of picocuries per gram

Results less than analytical limit indicated by a $(-)$. No entry indicates no analys is made. 


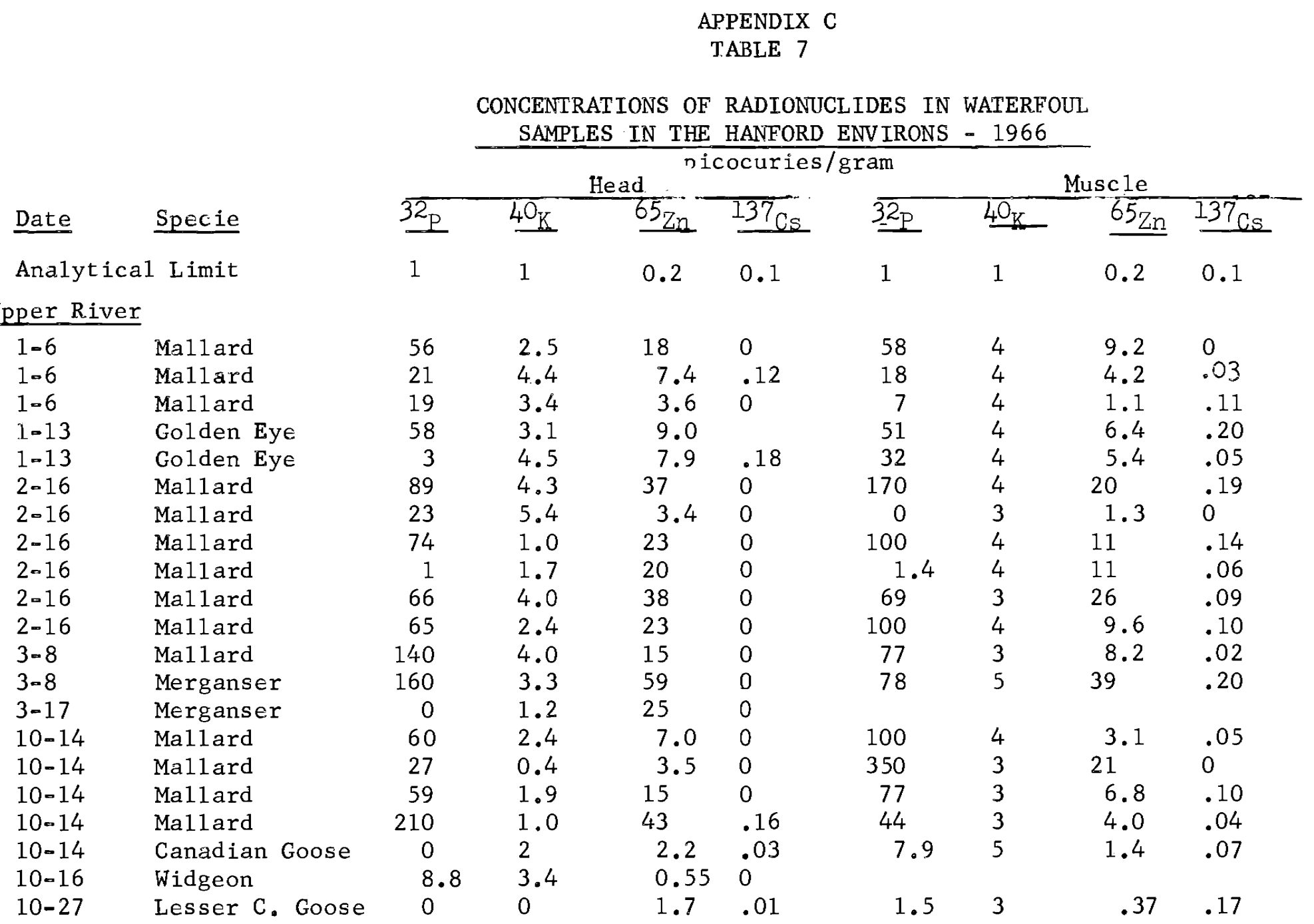

Results less than analytical limit are indicated by a $(-)$. No entry indicates no analysis made. 
APPENDIX C

TABLE 7 (Cont inued)

CONGENTRATIONS OE RADIONUCLIDES IN WATERFOUL SAMPLES IN THE HANFORD ENVTRONS - 1966

\begin{tabular}{|c|c|c|c|c|c|c|c|c|c|}
\hline \multirow[b]{3}{*}{ Date } & \multirow[b]{3}{*}{ Specie } & \multirow{2}{*}{\multicolumn{8}{|c|}{$\begin{array}{l}\text { picocuries/gram } \\
\text { Head }\end{array}$}} \\
\hline & & & & & & & & & \\
\hline & & $3 \overline{2}$ & $4 \mathrm{OK}_{\mathrm{K}}$ & $65_{7 n}$ & $137 \mathrm{Cs}$ & $32_{P}$ & $4 \mathrm{O}_{\mathrm{K}}$ & $65 \pi n$ & $137 \mathrm{cs}$ \\
\hline Analytical & Limit & 1 & 1 & 0.2 & 0.1 & 1 & 1 & 0.2 & 0.1 \\
\hline \multicolumn{10}{|l|}{ Upper River } \\
\hline $10-27$ & Lesser C. Goose & 4.2 & 1.3 & 2.2 & 0 & 0 & 3 & .21 & 0 \\
\hline $10-27$ & Lesser C. Goose & 0 & 1. 0 & 1.5 & .87 & 0 & 4 & .02 & 2.2 \\
\hline $10-27$ & Lesser C. Goose & 0 & 2.5 & 2.6 & .62 & 2.0 & 4 & 0 & 2.4 \\
\hline $10-27$ & Lesser C. Goose & 6.1 & .09 & 2.7 & .77 & $<1.0$ & 3 & .04 & 2.5 \\
\hline $10-27$ & Lesser C. Goose & 3.9 & 4.8 & 5.9 & .70 & 1.6 & 3 & .02 & 2.1 \\
\hline $10-27$ & Gadwal 1 & 120 & 0 & 5.8 & .29 & 2.5 & 2 & .25 & .87 \\
\hline $10-27$ & Gadwa11 & 5.5 & 4 & 2.9 & .21 & 6.1 & 3 & .34 & .56 \\
\hline $10-27$ & G. W. Teal & 960 & 0 & 151 & 0 & 240 & 6 & 50 & 0 \\
\hline $10-27$ & G.W. Teal & 290 & 9 & 82 & 0 & 190 & 6 & 28 & 1.2 \\
\hline $10-27$ & G. W. Teal & 440 & 5 & 30 & 0 & 340 & 5 & 5.7 & .09 \\
\hline $10=27$ & G. W. Teal & 340 & 8 & 46 & 0 & 330 & 8 & 26 & .01 \\
\hline $11-15$ & C. Honker & 61 & 3.1 & 24 & 0 & 49 & 3 & 14 & .09 \\
\hline $11-23$ & G.W. TeaL & 860 & 5.0 & 38 & .07 & 1400 & 7 & 22 & .31 \\
\hline $11-23$ & Golden Eye & 600 & 2.6 & 46 & .05 & 560 & 5 & 23 & .89 \\
\hline $11-23$ & Golden Eye & 0 & 4.7 & 0.38 & .27 & 1.5 & 3 & .13 &. .21 \\
\hline $11-23$ & Golden Eye & 4.6 & 3.2 & 0.49 & .10 & 1.0 & 4 & .13 & .15 \\
\hline $12-15$ & G. W. Tea1 & 27 & 1.1 & 3.5 & .19 & 1.0 & 3 & 0 & 0 \\
\hline $12-.15$ & G. W. Teal & 1.3 & 1.8 & .99 & .52 & 1.6 & 4 & 0.23 & .01 \\
\hline $12-14$ & Golden Eye & 2900 & 5 & 310 & 0 & 1600 & 4 & 91 & 0 \\
\hline $12-14$ & Golden Eye & 2600 & 5 & 260 & 0 & 900 & 7 & 3.3 &. .67 \\
\hline $12-14$ & Golden Eye & 1400 & 1 & 150 & 0 & 1400 & 5 & 81 & .11 \\
\hline $12-14$ & Golden Eye & 1000 & 2 & 130 & 0 & 1800 & 4 & 130 & 0 \\
\hline
\end{tabular}

Results less than analytical 1 imit are indicated by a (o). No entry indi cates no analysis made. 
APPENDIX C

TABLE 7 (Continued)

CONCENTRATIONS OF RADIONUCLIDES IN WATERFOUL

SAMPLES IN THE HANFORD ENVIRONS - 1966

Specie

Analytical Limit

$\begin{array}{ll}\text { Upper River } & \text { (Continued) } \\ 12-14 & \text { Canadian Goose } \\ 12-14 & \text { Canadian Goose } \\ 12-14 & \text { Canadian Goose } \\ 12-14 & \text { Canadian Goose } \\ 12-14 & \text { Lesser Goose }\end{array}$

\section{Lower River}

\begin{tabular}{|c|c|c|c|c|c|}
\hline $1-4$ & Mallard & & 4 & 3.7 & .15 \\
\hline $1-4$ & Mallard & 8 & 5 & 16 & 0 \\
\hline $1-4$ & Mallard & 7 & 3 & 29 & 0 \\
\hline $1-4$ & Mallard & 15 & 4 & 18 & .04 \\
\hline $1-4$ & Mallard & 6 & 1 & 0.9 & .11 \\
\hline 1.4 & Mallard & 15 & 4 & 18 & 0 \\
\hline $1-14$ & Mallard & 3 & 5 & 4.2 & .04 \\
\hline $1-14$ & Mallard & 100 & 5 & 18 & 0 \\
\hline $1-14$ & Mallard & 13 & 4 & 3.7 & .14 \\
\hline $1-14$ & Mallard & 47 & 3 & 22 & 0 \\
\hline $1-14$ & Mallard & 10 & 3 & 4.7 & .12 \\
\hline $1-14$ & Mallard & 20 & 5 & 17 & 0 \\
\hline $2-16$ & Mallard & .89 & 4 & 37 & 0 \\
\hline $2-16$ & Mallard & 23 & 5 & 3.4 & 0 \\
\hline $2-16$ & Mallard & 74 & $<1$ & 23 & 0 \\
\hline $2-16$ & Ma11ard & 1.4 & 2 & 19 & 0 \\
\hline $2-16$ & Mallard & 66 & 4 & 38 & 0 \\
\hline $2-16$ & Mal1ard & 65 & 2 & 23 & 0 \\
\hline $3-17$ & Merganser & 0 & 1 & 25 & 0 \\
\hline $3-17$ & Canadian Honker & $<1$ & & 38 & 0 \\
\hline
\end{tabular}

Results less than analytical limit are indicated by a (-). picocuries/gram

\begin{tabular}{l} 
picocuries /gram \\
Head \\
\hline $32 \mathrm{P}$ \\
\hline
\end{tabular}

$\begin{array}{llllllll}1 & 1 & 0.2 & 0.1 & 1 & 1 & 0.2 & 0.1\end{array}$

$\begin{array}{rrcrrrrr}10 & 2 & 28 & 0 & 12 & 3 & 20 & .13 \\ 1.3 & 2 & .98 & 0 & 1 & 3 & .12 & .03 \\ 0 & 2 & 1.4 & .05 & 2.5 & 3 & .26 & .03 \\ 45 & 4 & 7.3 & 0 & 1.0 & 3 & 3.2 & .07\end{array}$

No entry indicates no analysis made. 
APPENDIX $C$

TABLE 7 (Cont inued)

CONCENTRATIONS OF RADIONUCLIDES IN WATERFOUI

SAMPLES IN THE HANFORD ENV IRONS - 1966

\section{Date Specie \\ Analytical Limit}
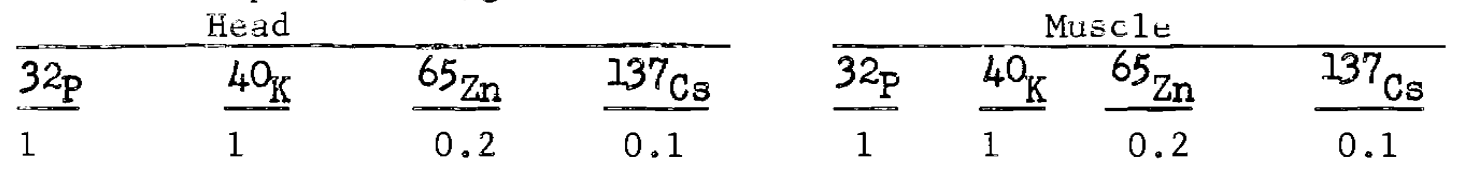

\begin{tabular}{ll} 
Lower River & (Continued) \\
\hline $10-6$ & Mallard \\
$10-6$ & Mallard \\
$10-6$ & Mallard \\
$10-6$ & Mallard \\
$10-6$ & Mallard \\
$10-24$ & Mallard \\
$10-24$ & Mallard \\
$10-24$ & Mallard \\
$10-24$ & Mallard \\
$10-24$ & Mallard \\
$10-24$ & Mallard \\
$10-24$ & Mallard \\
$10-24$ & Mallard \\
$10-24$ & Mallard \\
$10-31$ & Mallard \\
$10-31$ & Mallard \\
$10-31$ & Mallard \\
$10-31$ & Mallard \\
$10-31$ & Mallard \\
$10-31$ & Mallard \\
$10-31$ & Mallard \\
$10-31$ & Mallard \\
$10-31$ & Lesser C. Goose \\
$12-1$ & Mallard \\
$12-1$ & Mallard
\end{tabular}

$\begin{array}{cccc}210 & 1 & 17 & 0 \\ 260 & 3 & 21 & .04 \\ 60 & 3 & 18 & .05 \\ 47 & 0 & 0.58 & 0 \\ 3.1 & 2 & 1.6 & .03 \\ 6.3 & 3 & 3.7 & 0 \\ 8.6 & 3 & 6.7 & 0 \\ 1.10 & 1 & 25 & 0 \\ 7.2 & 2 & 3.5 & .08 \\ 150 & 1 & 13 & 0 \\ 200 & 1 & 18 & 0 \\ 220 & 0 & 0 & .07 \\ 5.1 & 2 & .77 & .04 \\ 7.4 & 3 & 1.8 & 0 \\ 62 & 3 & 7.6 & .13 \\ 63 & 3 & 8.5 & 0 \\ 80 & 4 & 8.5 & .20 \\ 39 & 5 & 3.1 & .09 \\ 100 & 2 & 6.0 & 0 \\ 6.4 & 3 & 2.5 & .10 \\ 13 & 3 & 8.7 & 0 \\ 150 & 0 & 7.4 & .25 \\ 0 & 1 & 5.4 & .01 \\ 0 & 2 & 2.9 & 0 \\ 3.0 & 2 & 3.9 & .22\end{array}$

Results less than analytical limit are indicated by a $(-)$. No entry indicates no analys is made.

$\begin{array}{cccc}310 & 4 & 8.0 & 0 \\ 290 & 3 & 9.5 & 0 \\ 100 & 3 & 6.4 & .03 \\ 33 & 3 & 3.5 & .04 \\ 29 & 3 & .23 & 0 \\ 3.4 & 4 & 1.5 & .01 \\ 6.9 & 4 & 2.9 & .05 \\ 210 & 4 & 6.2 & .22 \\ 6.3 & 3 & .63 & .15 \\ 130 & 4 & 6.7 & .23 \\ 1.5 & 3 & .08 & .02 \\ 200 & 4 & 2.9 & .03 \\ 280 & 3 & 3.9 & .04 \\ 1.1 & 3 & .18 & 0 \\ 31 & 4 & 4.0 & 0 \\ 30 & 3 & 3.3 & .12 \\ 24 & 4 & 3.3 & .04 \\ 19 & 4 & 1.0 & .02 \\ 90 & 3 & 2.1 & .02 \\ 1.4 & 3 & .62 & .09 \\ 16 & 4 & 3.9 & 0 \\ 6.1 & 4 & 1.1 & 1.3 \\ 0 & 3 & .14 & .09 \\ 1.0 & 4 & .76 & .07 \\ 8.6 & 3 & 2.2 & .11 \\ & & & \end{array}$


APPENDIX C

TABLE 7 (Continued)

CONCENTRATIONS OF RADIONUCLIDES IN WATERFOUL

SAMPLES IN THE HANFORD ENVIRONS - 1966

\section{Date $\quad$ Specie}

Analytical Limit

\section{Lower River (Continued}

\section{$12-1$ \\ Ma11 ard}

$12-1$

$12-1$

$12-1$

$12-8$

$12-8$

$12-8$

$12-8$

12-15

12-15

$12-15$

$12-15$

12-15

12-15

$12-15$

$12-15$

$12-15$

$12-15$

$12-15$

$12-20$

$12-20$

12-20

12-20

$12-20$

$12-20$

$12-24$

12-24

Mallard

Ma11ard

Mall ard

Mallard

Mallard

Ma11 ard

Mal1 ard

Mallard

Mallard

Mallard

Mallard

Mallard

Mallard

Ma11ard

Mallard

Merganser

Mallard

Golden Eye

Ma1lard

Mallard

Mallard

Mallard

Mallard

Malla rd

Mallard

Mallard

Results less than

No entry indicates no analysis made.

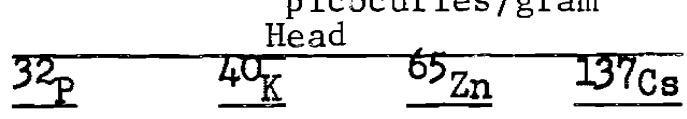

0.20 .1

$\begin{array}{cc}9.1 & 2 \\ 8.4 & 4 \\ 31 & 5 \\ 36 & 3 \\ 30 & 2 \\ 190 & 0 \\ 550 & 4 \\ 160 & 0 \\ 8.4 & 3 \\ 3.7 & 4 \\ 9.2 & 3 \\ 9.6 & 2 \\ 6.1 & 1 \\ 10 & 4 \\ 7.0 & 2 \\ 0 & 1 \\ 28 & 7 \\ 83 & 3 \\ 890 & 1 \\ 15 & 2 \\ 12 & 1 \\ 15 & 2 \\ 32 & 2 \\ 13 & 2 \\ 8.7 & 3 \\ 0 & 2 \\ 1.5 & 2\end{array}$

$\begin{array}{cl}5.0 & 0 \\ 3.2 & .09 \\ 13 & .15 \\ 23 & .05 \\ 20 & 0 \\ 48 & .17 \\ 160 & 0 \\ 31 & 0 \\ 1.0 & .06 \\ 5.7 & .23 \\ 10 & .03 \\ 8.9 & 0 \\ 5.6 & 0 \\ 7.8 & 0 \\ 10 & .11 \\ 11 & 0 \\ 31 & 0 \\ 17 & 0 \\ 160 & 0 \\ 14 & 0 \\ 4.4 & .08 \\ 6.6 & 0 \\ 3.4 & 0 \\ 3.3 & .02 \\ 5.5 & .01 \\ 10 & 0 \\ 7.5 & .03\end{array}$

$\begin{array}{cc}7.5 & .03\end{array}$

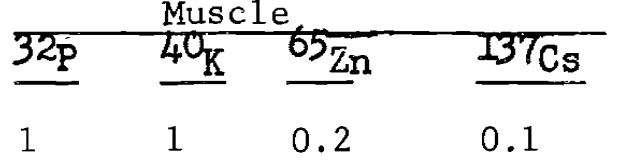

$\begin{array}{cc}14 & 3 \\ 9.4 & 3 \\ 18 & 4 \\ 79 & 4 \\ 26 & 2 \\ 100 & 3 \\ 11 & 3 \\ 920 & 4 \\ 0 & 3 \\ 5.4 & 3 \\ 4.4 & 3 \\ 9.1 & 3 \\ <.1 & 3 \\ 7.4 & 3 \\ 5.5 & 3 \\ 12 & 3 \\ 63 & 4 \\ 28 & 3 \\ 1500 & 3 \\ 5.2 & 3 \\ 18 & 2 \\ 15 & 3 \\ 7.1 & 3 \\ 4.9 & 3 \\ 12 & 3 \\ & 3 \\ & 3\end{array}$


APPENDIX C

TABLE 7 (Continued)

CONCENTRATIONS OF RADIONUCLIDES IN WATERFOUL

SAMPLES TN THE HANFORD ENVTRONS - 1966

\begin{tabular}{|c|c|c|c|c|c|c|c|}
\hline & $\begin{array}{r}\mathrm{pic} \\
\mathrm{He} a \mathrm{~d}\end{array}$ & ries/gr & & & Mis & & \\
\hline 320 & $4 O_{K}$ & $65 \mathrm{Zn}$ & $137 \mathrm{cs}$ & $32 \mathrm{p}$ & $40_{\mathrm{K}}$ & $65 \mathrm{Zn}$ & $13 T_{\mathrm{Cg}}$ \\
\hline 1. & 1 & 0.2 & 0.1 & 1. & 1 & 0.2 & 0.1 \\
\hline 44 & 2 & 14 & 0 & & 3 & & \\
\hline 8.6 & 2 & 7.5 & 0 & & 3 & & \\
\hline 28 & 3 & 13 & $a$ & & 3 & & \\
\hline 390 & 5 & 260 & 0 & & & & \\
\hline 330 & 5 & 49 & 0 & & & & \\
\hline 220 & 3 & 45 & 0 & & & & \\
\hline 28 & 19 & .05 & 0 & & & & \\
\hline 0 & 2.3 & 1.8 & & & & & \\
\hline 16 & 4.1 & .22 & & & & & \\
\hline 26 & 3.0 & $L G$ & & & & & \\
\hline 8 & 3.2 & .41 & .03 & & & & \\
\hline 41.0 & 3.1 & 91 & 0 & & & & \\
\hline 110 & 2.6 & 12 & 0 & & & & \\
\hline 280 & 3.8 & 85 & 0 & & & & \\
\hline 590 & 4.8 & 120 & 0 & & & & \\
\hline$c_{1}$ & 1.9 & 1.8 & .03 & $<1$ & 3.4 & .70 & .08 \\
\hline$<_{1}$ & 2.2 & 5.5 & .07 & 4.1 & 3.6 & 2.0 & .34 \\
\hline 6.6 & 2.7 & 4.7 & .03 & 7.2 & 3.6 & 1.4 & 11 \\
\hline$<1$ & 2.8 & .12 & .12 & 1 & 1.3 & .58 & 0 \\
\hline 480 & 2.2 & 87 & 0 & 560 & 3.6 & 40 & .09 \\
\hline 280 & 1.1 & 87 & & 460 & 4.0 & 43 & \\
\hline 350 & 4.0 & 81 & & 400 & 2.5 & 35 & \\
\hline 270 & 2.1 & 38 & & 330 & 2.9 & 24 & .06 \\
\hline 11 & 2.4 & .32 & 0 & $<1$ & 3.8 & .02 & 0 \\
\hline
\end{tabular}


APPENDIX C

TABLE 7 (Continued)

CONCENTRATIONS OF RADIONUCLIDES IN WATERFOUL

SAMPLES IN THE HANFORD ENVIRONS - 1966

\section{picocuries/gram}

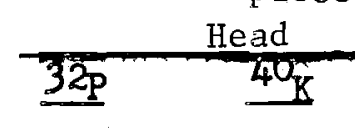

1

1
Date Specie

Analytical Limit

McNary (Cont inued)

\begin{tabular}{cl}
\hline $11-16$ & Golden Eye \\
$11-17$ & Honker \\
$11-17$ & Honker \\
Mesa & \\
\hline $10-16$ & Mallard \\
$10-16$ & Mallard \\
$10-16$ & Mallard \\
$10-16$ & Mallard \\
$12-18$ & Mallard \\
$12-18$ & Mallard \\
$12-18$ & Mallard \\
$12-18$ & Mallard \\
0 the110 & \\
\hline $12-18$ & Mallard \\
$12-18$ & Mallard \\
$12-18$ & Mallard \\
12018 & Mallard \\
$12-18$ & Mallard \\
$12-18$ & Mallard \\
$12-23$ & Mallard \\
$12-23$ & Mallard \\
$12-23$ & Mallard \\
$12-23$ & Mallard \\
$12-23$ & Mallard \\
12 & Mal
\end{tabular}

450
13
38

23

$\begin{array}{ll}.96 & 0 \\ .52 & 0\end{array}$

\section{7}

0

0$$
2
$$$$
3.5
$$$$
<1
$$$$
6.2
$$$$
5.7
$$

3.5
3.4
3.4

0.2

0.2 $137 \mathrm{cs}$

0.1

$\begin{array}{lcl}2.1 & 23 & 0 \\ 5.2 & .69 & 0 \\ 2.3 & 4.83 & .04 \\ 3.0 & 1.27 & .02 \\ 1.8 & 1.3 & .07 \\ 1.1 & 6.5 & 0 \\ 1.7 & 1.8 & .03 \\ 1.4 & 10.0 & 0\end{array}$

\section{0}

2.7

0
2.6

2.6
1.1 21 3.7 14

8.8

8.2

8.3

$\begin{array}{lll}1.1 & .05 & 0 \\ 2.0 & 6.2 & .03 \\ 1.0 & 0.30 & 0 \\ 1.8 & 8.2 & 0 \\ 2.5 & 4.5 & 0 \\ 1.6 & 12.3 & 0 \\ 1.8 & 0.28 & 0 \\ 1.4 & 0.17 & 0 \\ 1.0 & 2.4 & 0 \\ 1.0 & 3.1 & 0 \\ 2.5 & 1.3 & 0\end{array}$

Results less than analytical limit are indicated by (-). No entry indicates no analysis made.

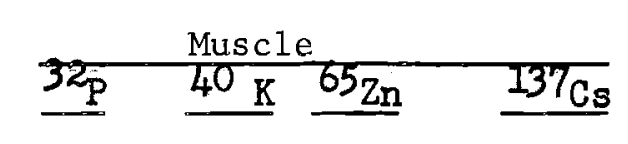

$\begin{array}{llll}280 & 3.2 & 9.4 & 0 \\ & 3.3 & .24 & 0 \\ 8.6 & 3.1 & .41 & .05\end{array}$ 
APPENDIX C

TABLE 7 (Continued)

CONCENTRATIONS OF RADIONUCLIDES IN WATERFOUL

SAMPLES IN THE HANFORD ENVIRONS - 1966 picocuries/gram

\section{Date $\underline{\text { Specie }}$}

Analytical Limit

Othello

12-26 Ma1lard

12-26 Mallard

12-26 Ma1lard

12-26 Mallard

12-26 Mallard

E1topia

12-14 Ma1lard

$12-14$

$12-14$

$12-14$

$12-14$

$12-14$

$12-14$

Wallula

$1-20$

$1-20$

Mallard

Mallard

Ma1lard

Mallard

Mallard

Ma1lard

Mallard

Mallard

Mallard

Island View

$\begin{array}{ll}2-4 & \text { Mallard } \\ 2-4 & \text { Malla rd } \\ 2-4 & \text { Mallard } \\ 2-4 & \text { Mallard } \\ 2-1 & \text { Mallard }\end{array}$

PaEtersoi.

2-18 Golden Eye

$2-18$

Golden Eye

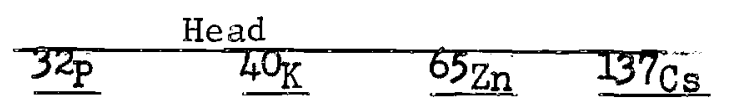

1

1

0.2

0.1

3.8
5.6
1.2
11
3.9

2.1

1.0

1.3

1.0

1.0

16

0.17

0.06

$.20 \quad 0.1$

5.5
2.4

20

4.4

11

1.1

1.0

6.40

2.6

1.1

1

1.8

1.9

1.2

1.5

13

.06

$7.6 \quad 0$

$7.0 \quad .01$

2.20

5.1

1.8

3.2

.04

$\begin{array}{rccl}5 & 3 & 11 & .06 \\ 1 & 2 & 3.3 & .13 \\ 12 & 8 & 15 & 0\end{array}$

No entry indicates no analysis made.

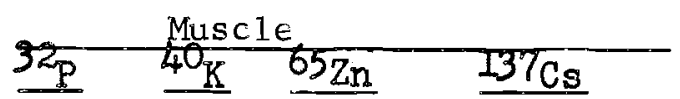

$0.2 \quad 0.1$

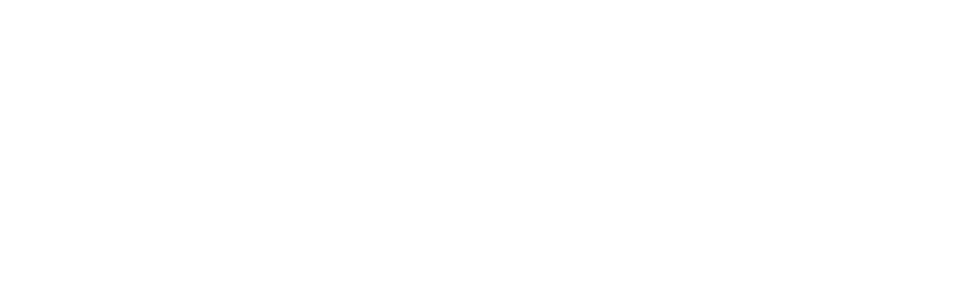


BNWL-439 APP

\section{APPENDIX D}

RADIONUCLIDES IN MARINE ORGANISMS 


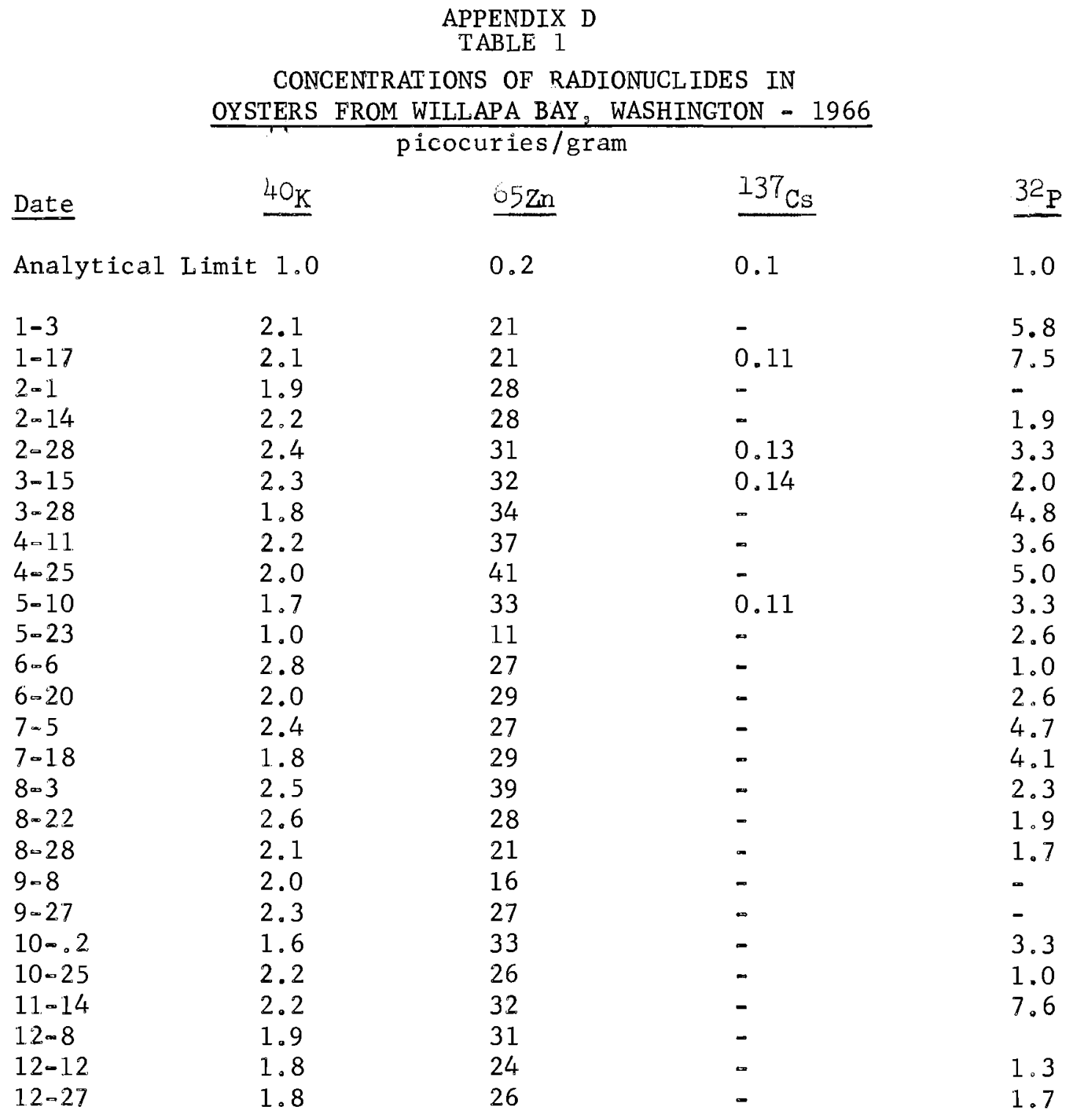

Results less than analytical limit are indicated by (-). No entry indicates no analysis made. 
BNWL-439 APP

\author{
APPENDIX E \\ RADIONUCLIDES IN THE ATMOSPHERE
}




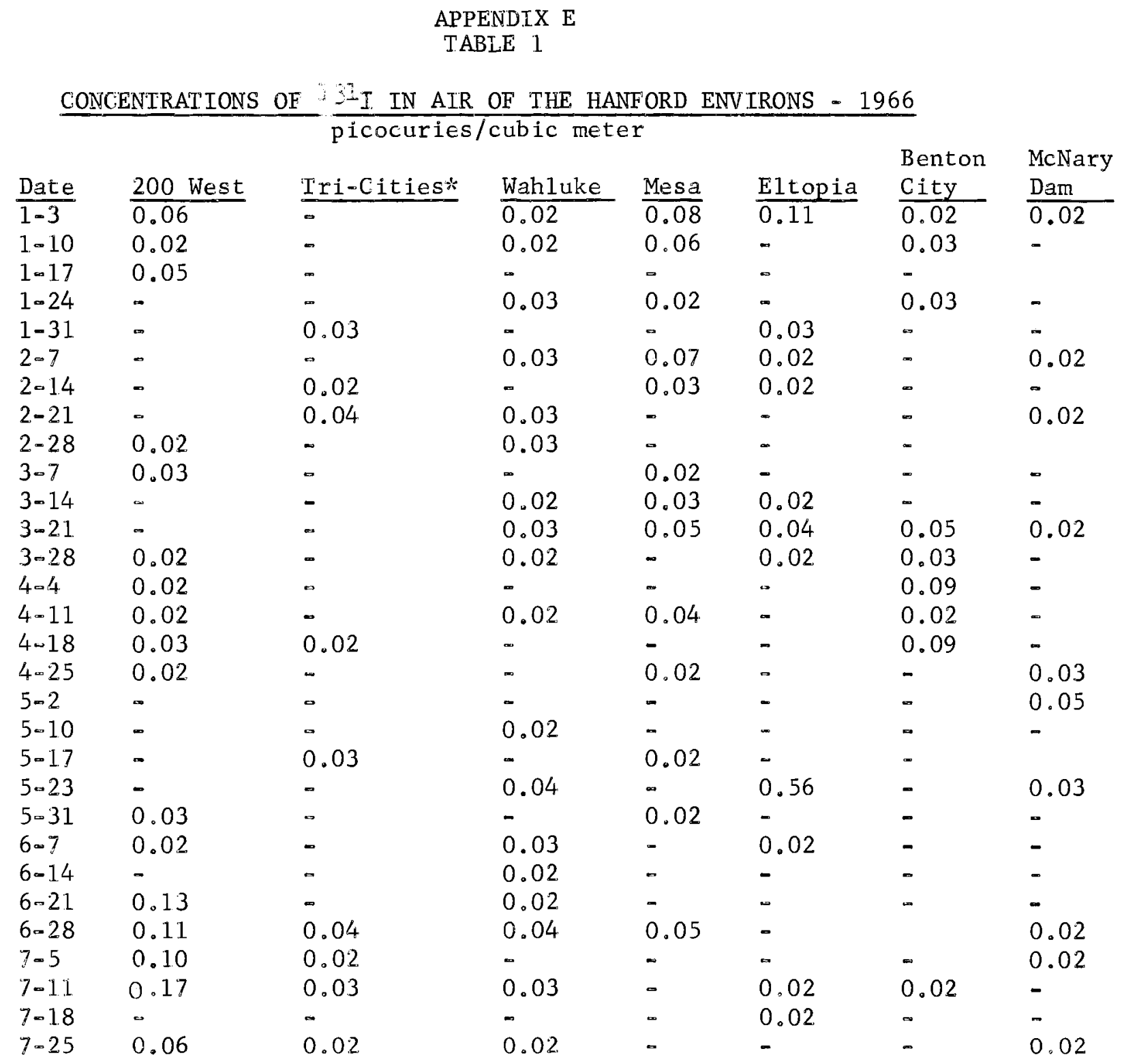

Results less than analyticy 1 imit of $0.02 \mathrm{pCi} / \mathrm{m}^{3}$ are indicated by a $(-)$. No entry indicates no analys is made. * Richland, Kennewick, and Pasco, Washington. 
APPENDIX E

TABLE 1 (Continued)

CONCENTRATIONS OF I3II IN AIR OF THE HANFORD ENVIRONS - 1966 picocuries/cubic meter

\begin{tabular}{|c|c|c|c|c|c|c|c|}
\hline Date & 200 West & Tri-Cities & Wahluke & Mesa & Eltopia & $\begin{array}{l}\text { Benton } \\
\text { City }\end{array}$ & $\begin{array}{l}\text { McNary } \\
\text { Dam }\end{array}$ \\
\hline $8-1$ & - & - & $\overline{-}$ & $\overline{-}$ & & 0.03 & - \\
\hline $8-8$ & 0.03 & & - & - & - & 0.02 & 0.02 \\
\hline $8-15$ & - & 0.02 & 0.02 & - & - & & 0.02 \\
\hline $8-22$ & 0.02 & - & - & - & - & 0.02 & 0.02 \\
\hline $8-29$ & - & & - & - & - & & 0.03 \\
\hline $9-5$ & - & - & 0.02 & 0.02 & & - & - \\
\hline $9-12$ & 0.02 & - & 0.02 & - & 0.04 & 0.02 & \\
\hline $9-19$ & - & - & - & - & & 0.02 & 0.02 \\
\hline $9-26$ & - & - & - & 0.02 & - & - & - \\
\hline $10-3$ & - & - & - & 0.02 & - & - & - \\
\hline $10-10$ & 0.03 & - & 0.05 & - & - & - & - \\
\hline $10-17$ & 0.03 & - & - & 0.02 & 0.02 & - & - \\
\hline $10-24$ & 0.02 & - & - & 0.02 & - & - & - \\
\hline $10-31$ & 0.03 & - & 0.03 & - & - & - & 0.02 \\
\hline $11-7$ & - & 0.03 & - & 0.04 & - & 0.02 & - \\
\hline $11-14$ & - & - & 0.02 & 0.02 & - & - & - \\
\hline $11-21$ & 0.02 & - & 0.03 & 0.03 & - & - & - \\
\hline $11-28$ & - & - & 0.03 & 0.02 & 0.04 & - & 0.05 \\
\hline $12-5$ & - & - & - & - & 0.02 & - & 0.02 \\
\hline $12-12$ & 0.02 & - & 0.02 & - & 0.12 & - & 0.02 \\
\hline $12-19$ & 0.04 & - & - & - & 0.03 & - & - \\
\hline $12-26$ & - & - & - & - & - & 0.02 & 0.03 \\
\hline
\end{tabular}

Results less than analytical limit of $0.02 \mathrm{pCi} / \mathrm{m}^{3}$ are indicated by a $(-)$. No entry indicates no analys is made.

* Richland, Kennewick, Pasco, Washington. 
APPENDIX E

CONCENTRATIONS OF RADIOACTIVE PART TCULATES

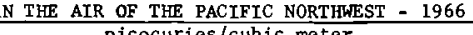

\begin{tabular}{l} 
Week \\
Ending Project \\
Date \\
\hline
\end{tabular} Tri-Cities*
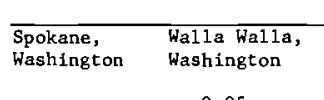

\begin{tabular}{|c|c|c|c|}
\hline $1-3$ & 0.36 & 0.05 & \\
\hline $1-10$ & 0.19 & 0.07 & 0.08 \\
\hline $1-17$ & 0.40 & 0.09 & 0.05 \\
\hline $1-24$ & 0.22 & 0.12 & 0.14 \\
\hline $1-31$ & 0.24 & 0.17 & 0.08 \\
\hline $2-7$ & 0.17 & 0.14 & 0.04 \\
\hline $2-14$ & 0.76 & 0.07 & 0.06 \\
\hline $\begin{array}{l}2-21 \\
2-28\end{array}$ & 0.31 & 0.09 & 0.08 \\
\hline $\begin{array}{l}2-28 \\
3-7\end{array}$ & 0.23 & 0.11 & \\
\hline $\begin{array}{l}3-7 \\
3-14\end{array}$ & 0.08 & 0.16 & 0.12 \\
\hline 3-14 & 0.25 & 0.10 & 0.12 \\
\hline $\begin{array}{l}3-21 \\
3-28\end{array}$ & 0.13 & 0.07 & 0.08 \\
\hline $4-4$ & $\begin{array}{l}0.32 \\
0.41\end{array}$ & $\begin{array}{l}0.21 \\
0.22\end{array}$ & 0.30 \\
\hline $4-11$ & 0.37 & $\begin{array}{l}0.22 \\
0.18\end{array}$ & $\begin{array}{l}0.24 \\
0.29\end{array}$ \\
\hline $4-18$ & 0.33 & 0.15 & 0.20 \\
\hline $4-25$ & 0.18 & 0.14 & 0.16 \\
\hline $5-2$ & 0.18 & 0.21 & 0.24 \\
\hline $5-10$ & 0.22 & 0.26 & 0.17 \\
\hline $5-17$ & 0.11 & 0.12 & 0.12 \\
\hline $5-23$ & 1.6 & 0.29 & 0.21 \\
\hline $5-31$ & 0.78 & 0.51 & 0.45 \\
\hline $6-7$ & 0.23 & 0.26 & \\
\hline $6-14$ & 0.57 & 0.41 & 0.6515 \\
\hline 6-21 & 0.29 & 0.33 & 0.31 \\
\hline $6-28$ & 0.34 & 0.21 & 0.21 \\
\hline $7-5$ & 0.24 & 0.18 & 0.14 \\
\hline $7-11$ & 0.32 & 0.28 & 0.32 \\
\hline $7-18$ & 0.29 & 0.22 & 0.37 \\
\hline $7-25$ & 0.31 & 0.15 & \\
\hline $8-1$ & 0.24 & 0.20 & 0.39 \\
\hline $8-8$ & 0.20 & 0.21 & 0.15 \\
\hline $8-15$ & 0.18 & 0.12 & 0.15 \\
\hline $8-22$ & 0.22 & 0.22 & 0.10 \\
\hline $8-29$ & 0.33 & 0.18 & 0.12 \\
\hline $9-5$ & 0.05 & 0.37 & 0.05 \\
\hline $9-12$ & 0.07 & 0.13 & 0.09 \\
\hline $9-19$ & 0.07 & 0.13 & 0.10 \\
\hline $9-26$ & 0.39 & 0.14 & 0.10 \\
\hline $10-3$ & 0.38 & 0.10 & 0.06 \\
\hline $10-10$ & 1.1 & 0.19 & 0.09 \\
\hline $10-17$ & 0.90 & 0.13 & \\
\hline $10-24$ & 0.88 & 0.11 & 0.04 \\
\hline $10-31$ & 0.32 & 0.19 & 0.04 \\
\hline $11-7$ & 1.2 & 0.54 & 0.42 \\
\hline $11-14$ & 0.33 & 0.24 & \\
\hline 11-21 & 0.15 & 0.13 & \\
\hline $11-28$ & 0.55 & 0.14 & 0.19 \\
\hline $12-5$ & 0.15 & 0.19 & 0,11 \\
\hline $12-12$ & 0.12 & 0.06 & 0.03 \\
\hline $12-19$ & 0.20 & 0.07 & 0.04 \\
\hline $12-26$ & 0.15 & 0.09 & \\
\hline
\end{tabular}

\begin{tabular}{|c|c|}
\hline \\
\hline $\begin{array}{l}0.05 \\
0.08\end{array}$ & \\
\hline & \\
\hline 0.16 & \\
\hline $\begin{array}{l}0.09 \\
0.05\end{array}$ & \\
\hline & \\
\hline $\begin{array}{l}0.09 \\
0.07\end{array}$ & \\
\hline & \\
\hline 0.06 & \\
\hline $\begin{array}{l}0.10 \\
0.10\end{array}$ & \\
\hline $\begin{array}{l}0.10 \\
0.22\end{array}$ & \\
\hline 0.16 & \\
\hline 0.11 & \\
\hline $\begin{array}{l}0.18 \\
0.16\end{array}$ & \\
\hline 0.23 & \\
\hline $0: 16$ & \\
\hline $\begin{array}{l}0.15 \\
1.9\end{array}$ & \\
\hline 0.26 & \\
\hline 0.35 & \\
\hline $\begin{array}{l}0.20 \\
0.39\end{array}$ & \\
\hline $\begin{array}{l}.39 \\
0.19\end{array}$ & \\
\hline 0.27 & \\
\hline $\begin{array}{l}0.25 \\
0.23\end{array}$ & \\
\hline 0.14 & \\
\hline 0.17 & \\
\hline $\begin{array}{l}0.12 \\
0.14\end{array}$ & \\
\hline 0.11 & \\
\hline 0.07 & \\
\hline $\begin{array}{l}0.07 \\
0.08\end{array}$ & \\
\hline 0.12 & \\
\hline 0.04 & \\
\hline $\begin{array}{l}0.08 \\
0.09\end{array}$ & \\
\hline 0.04 & \\
\hline 0.15 & \\
\hline 0.68 & \\
\hline 0.23 & \\
\hline $\begin{array}{l}0.16 \\
0.20\end{array}$ & \\
\hline $\begin{array}{l}0.20 \\
0.09\end{array}$ & \\
\hline 0.1 & \\
\hline 0.07 & \\
\hline
\end{tabular}

*Tri-Cities (Richland, Pasco and Kennewick, Washington)

\begin{tabular}{|c|c|c|c|c|c|c|c|}
\hline $\begin{array}{l}\text { akima, } \\
\text { ashingt on }\end{array}$ & $\begin{array}{l}\text { Washtucna, } \\
\text { Washington }\end{array}$ & $\begin{array}{l}\text { Pex } \\
\text { Wenatchee, } \\
\text { Hashington }\end{array}$ & $\begin{array}{l}\text { eter collaninit } \\
\text { Sunnyside, } \\
\text { Washington }\end{array}$ & $\begin{array}{l}\text { Es } \\
\text { E11ensburg, } \\
\text { Hashingt on }\end{array}$ & $\begin{array}{l}\text { Moses Lake, } \\
\text { Washington }\end{array}$ & $\begin{array}{l}\text { McNary Dam } \\
\text { Oregen }\end{array}$ & $\begin{array}{l}\text { Pendlet on } \\
\text { Oregon }\end{array}$ \\
\hline 0.05 & $\begin{array}{l}0.08 \\
0.07\end{array}$ & $\begin{array}{l}0.09 \\
0.05\end{array}$ & $\begin{array}{l}0.08 \\
0.05\end{array}$ & 0.06 & $\begin{array}{l}0.07 \\
0.08\end{array}$ & $\begin{array}{l}0.05 \\
0.05\end{array}$ & $\begin{array}{l}0.08 \\
0.07\end{array}$ \\
\hline 0.04 & & 0,11 & 0.10 & 0.03 & 0.10 & & 0.07 \\
\hline 0.09 & 0.10 & 0.13 & 0.10 & 0.08 & 0.17 & 0.09 & \\
\hline \multirow{3}{*}{0.07} & 0.12 & 0.08 & 0.18 & 0.08 & & 0.09 & 0.08 \\
\hline & 0.09 & & 0.07 & 0.05 & 0.07 & 0.07 & \\
\hline & & 0.05 & 0.08 & 0.05 & 0.11 & 0.06 & \\
\hline 0.07 & & 0.09 & $\begin{array}{l}0.10 \\
0.07\end{array}$ & 0.07 & $\begin{array}{l}0.09 \\
0.08\end{array}$ & 0.11 & 0.07 \\
\hline \multirow[t]{2}{*}{0.07} & 0.07 & 0.08 & 0.15 & & 0.12 & 0.04 & 0.11 \\
\hline & 0.12 & 0.07 & 0.11 & 0.05 & 0.11 & 0.05 & 0.24 \\
\hline 0.06 & 0.13 & 0.08 & 0.06 & 0.07 & & 0.06 & 0.16 \\
\hline 0.29 & 0.13 & 0.10 & 0.23 & 0.07 & 0.15 & 0.09 & 0.26 \\
\hline \multirow{6}{*}{$\begin{array}{l}0.14 \\
0.09\end{array}$} & 0.24 & 0.22 & 0.18 & 0.14 & 0.22 & 0.18 & 0.17 \\
\hline & 0.17 & 0.19 & 0.17 & 0.10 & 0.11 & 0.17 & 0.16 \\
\hline & $\begin{array}{l}0.14 \\
0.15\end{array}$ & 0.13 & $\begin{array}{l}0.14 \\
0.14\end{array}$ & 0.12 & $\begin{array}{l}0.27 \\
0.32\end{array}$ & $\begin{array}{l}0.10 \\
0.10\end{array}$ & $\begin{array}{l}0.20 \\
0.13\end{array}$ \\
\hline & 0.17 & 0.15 & 0.16 & 0.13 & 0.24 & $\begin{array}{l}.10 \\
0.12\end{array}$ & $\begin{array}{l}0.13 \\
0.22\end{array}$ \\
\hline & 0.22 & 0.20 & 0.20 & 0.08 & 0.22 & 0.13 & 0.22 \\
\hline & 0.19 & 0.15 & 0.10 & 0.12 & 0.14 & 0.13 & 0.08 \\
\hline \multirow[t]{2}{*}{0.55} & 0.32 & 0.36 & 0.14 & 0.31 & 0.60 & 0.67 & 0.50 \\
\hline & 0.47 & 0.25 & 0.44 & 0.14 & 0.73 & 0.27 & 0.33 \\
\hline \multirow{4}{*}{0.23} & 0.54 & 0.40 & 0.21 & 0.14 & 0.29 & 0.68 & 0.92 \\
\hline & 0.54 & 0.74 & 0.27 & 0.22 & 0.27 & 0.25 & 0.35 \\
\hline & 0.36 & 0.24 & 0.24 & 0.15 & 0.18 & 0.33 & 0.34 \\
\hline & 0.19 & 0.18 & 0.22 & 0.12 & 0.20 & 0.18 & 0.17 \\
\hline \multirow{2}{*}{0.16} & 0.26 & 0.21 & 0.13 & & 0.28 & 0.17 & 0.34 \\
\hline & 0.31 & 0.21 & 0.22 & 0.16 & & 0.23 & \\
\hline \multirow{2}{*}{0.29} & 0.26 & 0.25 & 0.24 & & & 0.16 & 0.22 \\
\hline & 0.25 & 0.12 & 0.28 & 0.13 & 0.23 & & 0.16 \\
\hline \multirow{7}{*}{$\begin{array}{l}0.14 \\
0.12\end{array}$} & & $\begin{array}{l}0.13 \\
0.18\end{array}$ & $\begin{array}{l}0.15 \\
0.15\end{array}$ & & $\begin{array}{l}0.19 \\
0.18\end{array}$ & 0.15 & 0.17 \\
\hline & & $\begin{array}{l}0.18 \\
0.15\end{array}$ & & $\begin{array}{l}0.23 \\
0.13\end{array}$ & $\begin{array}{l}0.18 \\
0.18\end{array}$ & 0.11 & 0.14 \\
\hline & 0.29 & $\begin{array}{l}0.13 \\
0.14\end{array}$ & 0.06 & 0.07 & $\begin{array}{l}.18 \\
0.12\end{array}$ & $\begin{array}{l}0.14 \\
0.13\end{array}$ & $\begin{array}{l}0.15 \\
0.09\end{array}$ \\
\hline & & 0.09 & 0.00 & 0.10 & 0.06 & $\begin{array}{l}0.13 \\
0.10\end{array}$ & $\begin{array}{l}0.09 \\
0.07\end{array}$ \\
\hline & 0.08 & 0.07 & 0.05 & 0.05 & 0.07 & 0.08 & 0.09 \\
\hline & 0.08 & 0.09 & 0.06 & & 0.10 & 0.04 & 0.06 \\
\hline & & 0.08 & 0.05 & & 0.08 & 0.09 & 0.13 \\
\hline \multirow[t]{4}{*}{0.03} & & 0.09 & 0.04 & 0.04 & 0.20 & 0.05 & 0.06 \\
\hline & & 0.08 & 0.09 & 0.04 & 0.11 & 0.08 & 0.07 \\
\hline & 0.07 & 0.07 & 0.05 & 0.04 & 0.09 & 0.11 & 0.09 \\
\hline & 0.07 & 0.08 & 0.08 & 0.06 & 0.11 & 0.05 & 0.06 \\
\hline 0.05 & 0.11 & 0.05 & 0.03 & 0.03 & 0.05 & 0.05 & \\
\hline 0.04 & 0.05 & 0.04 & 0.05 & 0.05 & 0.25 & & 0.05 \\
\hline 0.53 & & 0.65 & 0.08 & 0.34 & & 0.11 & 0.83 \\
\hline 0.40 & & 0.13 & 0.29 & 0.28 & 0.17 & 0.56 & 0.22 \\
\hline $\begin{array}{l}0.12 \\
0.14\end{array}$ & & 0.10 & & 0.09 & 0.26 & 0.16 & 0.13 \\
\hline $\begin{array}{l}.14 \\
0.24\end{array}$ & & 0.10 & 0.05 & 0.14 & & $\begin{array}{l}0.11 \\
0.10\end{array}$ & 0.15 \\
\hline 0.11 & 0.12 & 0.10 & 0.08 & & 0.34 & 0.08 & 0.06 \\
\hline 0.05 & & 0.10 & & & 0.07 & 0.06 & 0.05 \\
\hline 0.05 & & 0.05 & & 0.03 & 0.07 & 0.08 & 0.08 \\
\hline
\end{tabular}


BNWL-439 APP

\section{APPENDIX F}

RADIONUCLIDES IN FARM PRODUCE

AND COMMERCIAL FOODSTUFFS 


\section{APPENDIX F}

TABLE 1

\section{CONCENTRATIONS OF RADIONUCLIDES IN MILK - 1966}

$$
\text { picocuries/liter }
$$

\begin{tabular}{|c|c|c|c|c|c|c|}
\hline Date & 131 & $40_{K}$ & $05 \mathrm{Zn}$ & $137 \mathrm{Cs}$ & $32_{\mathrm{F}}$ & $90 \mathrm{Sr}$ \\
\hline $\begin{array}{l}\text { Analytical } \\
\text { Limit }\end{array}$ & 3 & 400 & 100 & 30 & 200 & 2 \\
\hline \multicolumn{7}{|c|}{ Riverview Irrigation District } \\
\hline $1-4$ & 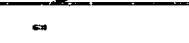 & 1500 & 180 & - & & 5.8 \\
\hline $1=11$. & - & 1300 & 150 & - & & \\
\hline $1-18$ & - & 1600 & 150 & $\infty$ & & \\
\hline $1-25$ & - & 1500 & 140 & 35 & & \\
\hline $2=1$ & 4 & 1500 & 160 & 36 & & \\
\hline $2-8$ & - & 1400 & 150 & - & & \\
\hline $2-15$ & 3 & 1500 & 140 & - & & \\
\hline $2=2 ?$ & $=$ & 1300 & 150 & - & & \\
\hline $3-1$ & - & 1400 & 120 & - & & \\
\hline $3-8$ & $=$ & & & & - & \\
\hline $3-15$ & - & 1100 & 120 & - & & \\
\hline $3-22$ & - & 91.0 & - & - & & \\
\hline $3-29$ & - & 1200 & - & - & & \\
\hline $4-5$ & $\infty$ & 1300 & 100 & - & & \\
\hline $4-12$ & - & 1200 & - & 30 & - & \\
\hline $4-19$ & - & 1200 & - & - & & \\
\hline $4-26$ & - & 1300 & 120 & - & & \\
\hline $5-3$ & $\infty$ & 1400 & 100 & 31 & & \\
\hline $5-10$ & - & 1300 & 290 & - & 1200 & \\
\hline $5-17$ & - & 1300 & 500 & - & & \\
\hline $5-24$ & - & 1200 & 570 & $\infty$ & & \\
\hline $5-31$ & 10 & 1400 & 670 & - & & \\
\hline $6-7$ & 6 & 1300 & 610 & 42 & 950 & \\
\hline $6-14$ & 5 & 1500 & 630 & 34 & & \\
\hline $6-21$ & - & 1500 & 590 & - & & \\
\hline $6-28$ & 3 & 1300 & 520 & - & & \\
\hline $7=5$ & 4 & 1500 & 550 & - & & 4.8 \\
\hline $7-12$ & 6 & 1400 & 580 & - & 870 & \\
\hline $7-19$ & 4 & 1400 & 590 & - & & \\
\hline $7-26$ & 3 & 1500 & 600 & - & & \\
\hline $8-4$ & - & 1400 & 490 & - & & \\
\hline $8-9$ & $=$ & 1300 & 550 & - & 980 & \\
\hline $8-16$ & - & 1300 & 480 & $\approx$ & & \\
\hline $8-23$ & & 1500 & 520 & - & & \\
\hline $8-30$ & - & 1400 & 380 & - & & \\
\hline
\end{tabular}

Results less than analytical limit are indicated by a $(\infty)$. No entry indicates no analysis made. 


\section{APPENDIX F}

TABLE 1 (Continued)

\section{CONCENTRATIONS OF RADIONUCLIDES IN MILK - 1966} picocuries/liter

\begin{tabular}{|c|c|c|c|c|c|c|}
\hline Date & ${ }^{131_{I}}$ & $4 \mathrm{O}_{\mathrm{K}}$ & $05 \mathrm{zn}$ & $137 \mathrm{Cs}$ & $32_{P}$ & $90_{\mathrm{Sr}}$ \\
\hline $\begin{array}{l}\text { Analytical } \\
\text { Limit }\end{array}$ & 3 & 400 & 100 & 30 & 200 & 2 \\
\hline
\end{tabular}

Riverview Irrigation District

\begin{tabular}{llllll}
\hline $9-6$ & - & 1400 & 440 & - & \\
$9-13$ & 3 & 1400 & 490 & - & 570 \\
$9-20$ & 3 & 1400 & 450 & - & \\
$9-27$ & - & 1200 & 400 & - & \\
$10-4$ & - & 1400 & 370 & - & \\
$10-11$ & - & 1200 & 360 & - & 1200 \\
$10-18$ & - & 1400 & 570 & - & \\
$10-25$ & - & 1300 & 640 & - & \\
$11-1$ & - & 1500 & 540 & - & \\
$11-8$ & 4 & 1400 & 500 & - & 620 \\
$11-15$ & - & 1300 & 300 & - & \\
$11-22$ & - & 1200 & & - & \\
$11-29$ & 3 & 1600 & 240 & - & \\
$12-6$ & - & 1400 & 180 & - & \\
$12-13$ & - & 1400 & 130 & - & - \\
$12-20$ & - & 1200 & 110 & - & - \\
$12-27$ & - & 1300 & 130 & - &
\end{tabular}

Ringold Farm No. 1

\begin{tabular}{llllll}
\hline $3-15$ & - & 1200 & 320 & - & \\
$3-22$ & - & 1100 & 270 & - & \\
$3-29$ & - & 1300 & 320 & - & \\
$4-5$ & - & 1300 & 240 & - & \\
$4-12$ & 4 & 1300 & 180 & - & - \\
$4-19$ & - & 1400 & 250 & - & \\
4026 & - & 1600 & 260 & - & \\
$5-3$ & - & 1400 & 270 & - & 500 \\
$5-10$ & - & 1400 & 240 & - & \\
$5-17$ & - & 1400 & 370 & - & \\
$5-24$ & - & 1600 & 490 & - & \\
$5-31$ & 3 & 1400 & 490 & - & \\
$6-7$ & 4 & 1300 & 290 & - & - \\
$6-14$ & 5 & 1800 & 430 & 40 & \\
$6-21$ & - & 1500 & 620 & - & \\
$6-28$ & 6 & 1400 & 380 & - &
\end{tabular}

Results less than analytical limit are indicated by $(-)$. No entry indicates no analys is made. 
APPENDIX $\mathrm{F}$

TABLE 1 (Continued)

CONCENTRATIONS OF RADIONUCLIDES IN MILK - 1966 picocuries/liter

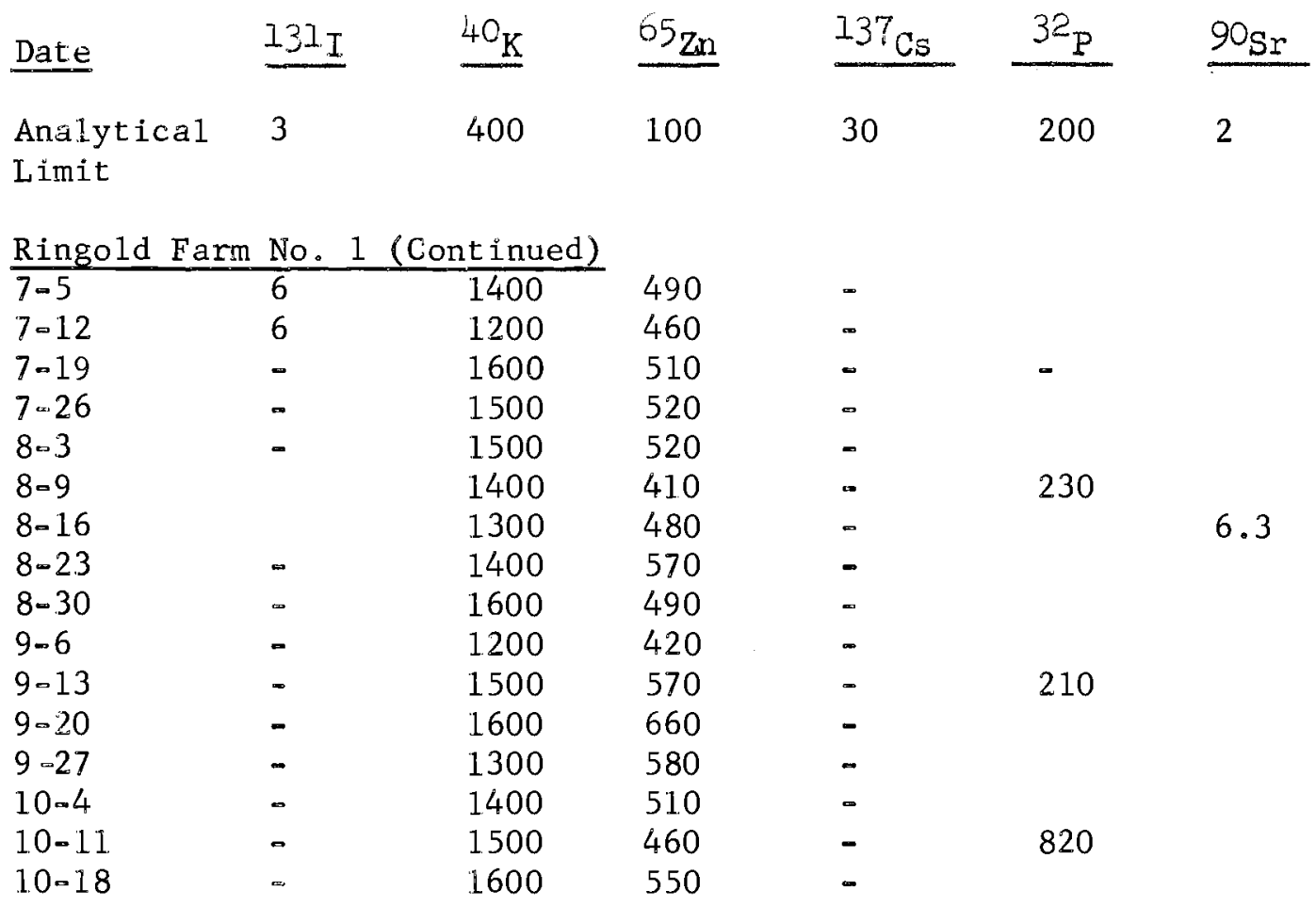

Ringold Farm No. 2

\begin{tabular}{lrrrll}
\hline $9-13$ & & 1300 & 300 & - & 1100 \\
$10-25$ & 5 & 1200 & 230 & - & \\
$10-27$ & 5 & & & & \\
$11-1$ & 6 & & & & \\
$11-3$ & 5 & & & \\
$11-8$ & 13 & & & \\
$11-10$ & 10 & & & & \\
$11-15$ & 7 & & & & \\
$11-22$ & 5 & 1400 & 100 & - & \\
$11-29$ & 4 & & & \\
$12-6$ & 4 & & & \\
$12-13$ & - & & & & \\
$12-20$ & - & 1300 & - & - & \\
$12-27$ & - & 940 & - & -
\end{tabular}

Results less than analytical limit are indicated by a $(-)$. No entry indicates no analysis made. 
APPENDIX F

TABLE 1 (Continued)

CONCENTRATIONS OF RADIONUCLIDES IN MILK - 1966 picocuries/1iter

\begin{tabular}{|c|c|c|c|c|c|c|}
\hline Date & $131_{I}$ & ${ }^{40} \mathrm{~K}$ & $65 \mathrm{zn}$ & $137_{\mathrm{Cs}}$ & $32_{p}$ & $90_{\mathrm{Sr}}$ \\
\hline $\begin{array}{l}\text { Analytical } \\
\text { Limit }\end{array}$ & 3 & 400 & 100 & 30 & 200 & 2 \\
\hline
\end{tabular}

$\frac{\text { Pasco Farm No. } 1}{1-5}$

$1-11$
$1-18$
$1-25$
$2-1$
$2-8$
$2-15$
$5-10$
$5-12$
$5-16$
$5-17$
$5-18$
$5-19$
$5-24$
$5-31$
$6-7$

6-7

$6-14$

6-21

$6-28$

$7-5$

$7-13$

$7-19$

$7-27$

$8-3$

8-9

$8-16$

$8-23$

$8-30$

9-6

$9-13$

9-20

9-27

$10-4$

$10-11$

$10-18$

$10-24$

10-25

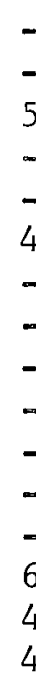

1300

1300

$-$

$-$

5

$-$

$\overline{4}$

4

$\begin{array}{lll}- & 1300 \quad 270\end{array}$

-

$-$

1500

160

1100

1100

37

2.6

1400

1200

1200

1400

1500

1200

1400

1200

1200

1200

1200

1300

1200

980

1200

1300

1100

1200

1200

1200

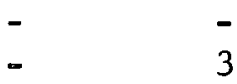

$-$

$-$

$-$

-

$-$

$-$

$-$

(2) 
APPENDIX F

TABLE 1 (Continued)

$\frac{\text { CONCENTRATIONS OF RADIONUCLIDES IN MILK }-1966}{\text { picocuries/1iter }}$

\begin{tabular}{|c|c|c|c|c|c|c|}
\hline Date & I3II & $4 \mathrm{O}_{\mathrm{K}}$ & $65 \mathrm{zn}$ & $137 \mathrm{Cs}$ & $32_{p}$ & $90_{\mathrm{Sr}}$ \\
\hline $\begin{array}{l}\text { Analytical } \\
\text { Limit }\end{array}$ & 3 & 400 & 100 & 30 & 200 & 2 \\
\hline \multicolumn{7}{|c|}{ Pasco Farm No. 1 (Continued) } \\
\hline $10-27$ & 3 & & & & & \\
\hline $10-31$ & - & & & & & \\
\hline $11-1$ & - & 1100 & - & - & & \\
\hline 11.5 & 3 & & & & & \\
\hline $11-7$ & - & & & & & \\
\hline $11-8$ & - & 1200 & - & - & 4000 & \\
\hline $11-10$ & 5 & & & & & \\
\hline $11=15$ & - & 1400 & - & - & & \\
\hline $11-22$ & - & 970 & - & - & & \\
\hline $11-29$ & 3 & 1200 & - & - & & \\
\hline $12-6$ & - & 1100 & - & - & & 2.0 \\
\hline $12-13$ & - & 1000 & - & - & - & \\
\hline $12-20$ & - & 510 & - & - & 2600 & \\
\hline $12-27$ & - & 900 & - & - & & \\
\hline
\end{tabular}

Pasco Farm No. 2

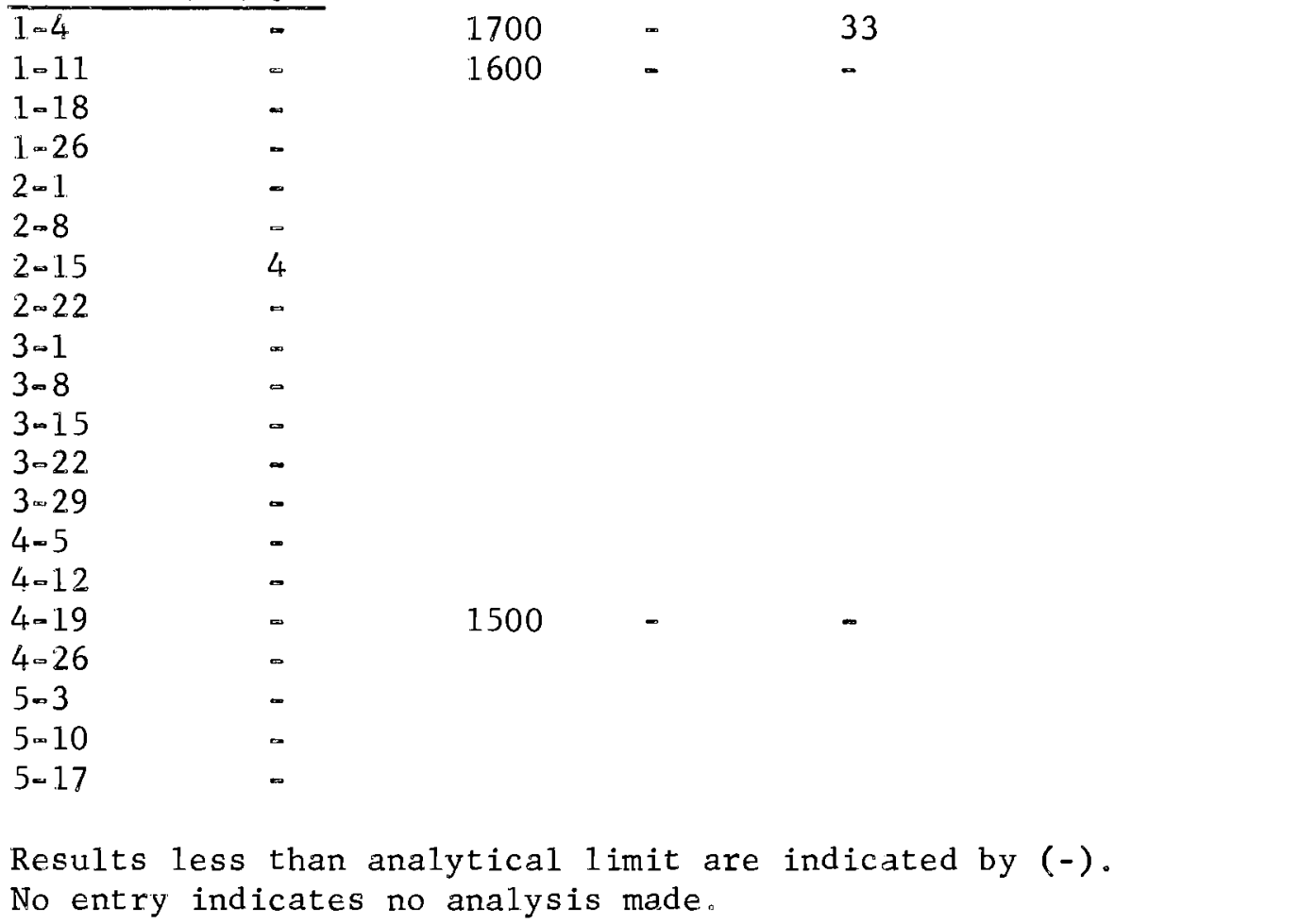


APPENDIX $\mathrm{F}$

TABLE 1 (Continued)

\section{$\frac{\text { CONCENTRATIONS OF RADIONUCLIDES IN MILK - } 1966}{\text { picocuries/1iter }}$}

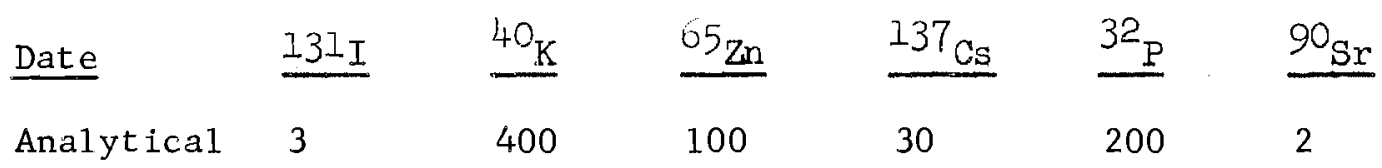

Limit

Pasco Farm No. 2 (Continued)

$5-24$

$5-31$

$6-7$

$6-14$

$6-21$

$6-28$

$7-5$

$7-12$

$7-19$

$7-27$

8- 3

1300

8-9

1500

$8 \sim 16$

$8-23$

$8-30$

$9-6$

9- 13

9-20

$9-27$

10-4

10-11

$10-18$

10- 25

$11-1$

$11-8$

$11-1.5$

$11-22$

$11-29$

$12-6$

12-13

$12-20$

$12-27$

6

3

-

-

-

11

$\begin{array}{rrr}1300 & - & - \\ 1500 & - & -\end{array}$

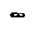

-

$990 \quad-\quad$ -

$-$

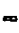

$-$

$-$

$\rightarrow$

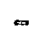

$-$

$-$

$-$

$-$

5

4

$-$

-

$\infty$

$-$

$-$

E1topia

$1-4$

1- 12

$1-18$
1700

1400

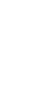

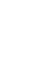

Results less than analytical limit are indicated by a (-). No entry indicates no analysis made. 
APPENDIX F

TABLE 1 (Continued)

\section{$\frac{\text { CONCENTRATIONS OF RADIONUCLIDES IN MILK - } 1966}{\text { picocuries/1iter }}$}

\begin{tabular}{|c|c|c|c|c|c|c|}
\hline Date & $131 I$ & $40_{K}$ & $65 \mathrm{Zn}$ & $137_{\mathrm{Cs}}$ & $32_{F}$ & ${ }^{90} \mathrm{Sr}$ \\
\hline Analytical & 3 & 400 & & 30 & & 2 \\
\hline
\end{tabular}

Limit

Eltopia (Continued)

$1-26$

$2-1$

2-9

3

$2-9$

$2-23$

$3-1$

$3-9$

$3-15$

$3-23$

$3-29$

4-6

$4-1.2$

4-20

$4-26$

$5-4$

$5-10$

$5-18$

$5-24$

$6-1$

$6-7$

$6-14$

$6-21$

$6-28$

$7=5$

$7-12$

$7-1.9$

$7-26$

$8-4$

$8-9$

$8-16$

$8-23$

$8-30$

$9-6$

$9-13$

$9-20$

$9-27$

8.6

4.2

Results less than analytical limit are indicated by a ( ) . No entry indicates no analysis made. 
APPENDIX F

TABLE 1 (Continued)

\section{CONCENTRATIONS OF RADIONUCLIDES IN MILK - 1966}

$$
\text { picocuries/liter }
$$

\begin{tabular}{|c|c|c|c|c|c|c|}
\hline Date & ${ }^{1} 3 I_{I}$ & $4 O_{K}$ & $65 \mathrm{zm}$ & $137_{\mathrm{Cs}}$ & $32_{P}$ & $90_{\mathrm{SI}}$ \\
\hline \multicolumn{7}{|c|}{ Analytical } \\
\hline Limit & 3 & 400 & 100 & 30 & 200 & 2 \\
\hline \multicolumn{7}{|c|}{ E1topia (Continued) } \\
\hline $10-4^{2}$ & - & & & & & \\
\hline $10-11$ & - & & & & & \\
\hline $10-18$ & $\infty$ & & & & & \\
\hline $10-25$ & 7 & & & & & \\
\hline $11-1$ & - & 1300 & - & - & & 2.9 \\
\hline $11-8$ & 13 & & & & & \\
\hline $11-17$ & 10 & & & & & \\
\hline $11-22$ & 8 & & & & & \\
\hline $11-29$ & 3 & & & & & \\
\hline $12-6$ & - & & & & & \\
\hline $12-13$ & - & & & & & \\
\hline $12-20$ & - & & & & & \\
\hline $12=27$ & $=$ & & & & & \\
\hline
\end{tabular}

West Richland Farm No。 1

$\begin{array}{lllll}1-6 & - & 1400 & - & - \\ 1-13 & - & 1500 & - & - \\ 1-20 & - & 1200 & - & - \\ 1-27 & 4 & 1300 & - & - \\ 2-3 & - & 1500 & - & - \\ 2-17 & - & 1700 & - & 33 \\ 3-3 & - & 1400 & - & - \\ 3-10 & - & 1100 & - & - \\ 3-17 & - & 1300 & - & - \\ 3-25 & - & 1600 & - & - \\ 3-31 & - & 1300 & - & - \\ 6-23 & 3 & 1300 & - & - \\ 6-30 & 3 & 1500 & - & 30 \\ 7-7 & - & 1500 & - & 42 \\ 7-14 & 4 & 1500 & - & 37 \\ 7-28 & - & 1500 & - & 32 \\ 8-4 & - & 1200 & - & - \\ 8-11 & - & 1300 & - & - \\ 8-18 & - & 1400 & - & -\end{array}$

Results less than analytical 1 imit are indicated by a $(-)$. No entry indicates no analysis made. 


$$
\begin{aligned}
& \text { APPENDIX } \mathrm{F} \\
& \text { TABLE } 1 \text { (Cont inued) }
\end{aligned}
$$

$\frac{\text { CONCENTRATIONS OF RADIONUCLIDES IN MLLK - } 1966}{\text { picocuries/liter }}$

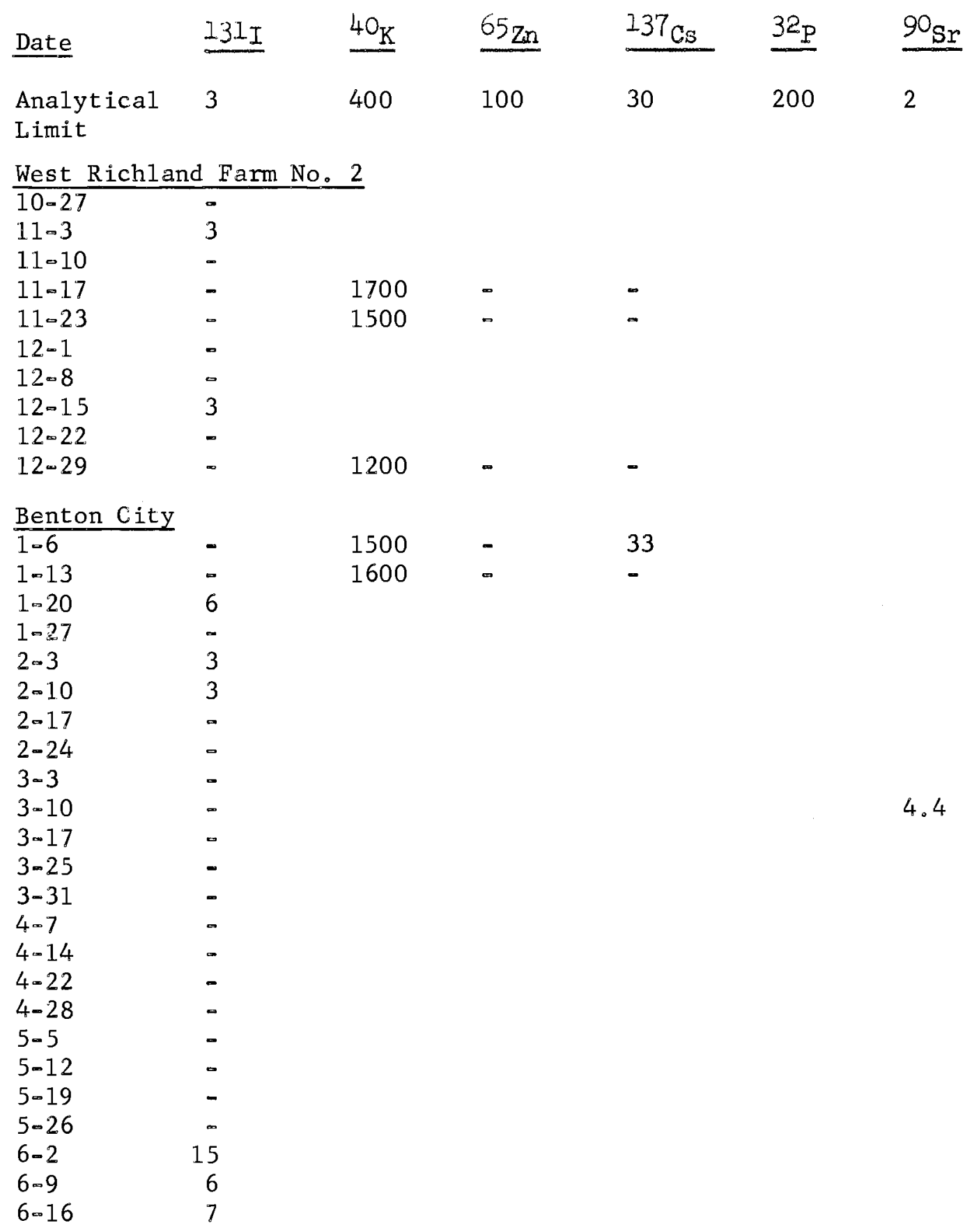

Results less than analytical 1 imit are indicated by a $(-)$. No entry indicates no analysis made. 


\section{APPENDIX F}

TABLE 1 (Continued)

\section{$\frac{\text { CONCENTRATIONS OF RADIONUCLIDES IN MILK }-1966}{\text { picocuries/1iter }}$}

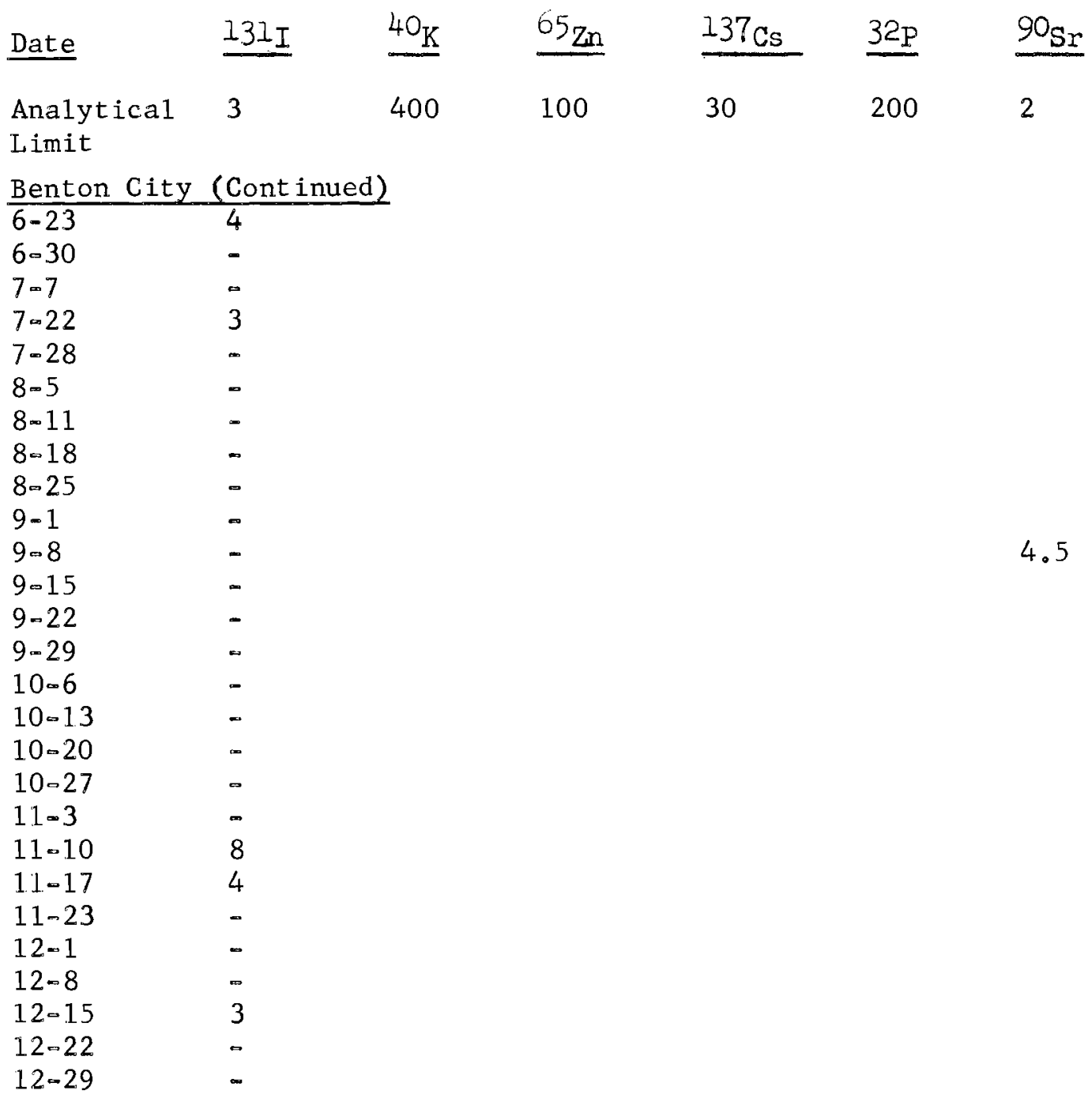

Milk Shed Samples

\begin{tabular}{lrlll}
\multicolumn{2}{l}{ Co1umbia } & Basin \\
\hline $1-13$ & 7 & 1300 & 110 & - \\
$1-27$ & - & 1500 & - & - \\
$2-10$ & - & 1400 & - & - \\
$2-23$ & - & 1200 & - & 31
\end{tabular}

Results less than analytical limit are indicated by a $(-)$. No entry indicates no analys is made. 
APPENDIX F

TABLE 1 (Continued)

CONCENTRATIONS OF RADIONUCLIDES IN MILK - 1966

picocuries/1iter

\begin{tabular}{|c|c|c|c|c|c|c|}
\hline Date & ${ }^{13} I_{I}$ & $40_{K}$ & $65 \mathrm{zn}$ & $137_{\mathrm{Cs}}$ & $32_{P}$ & $90_{\mathrm{Sr}}$ \\
\hline $\begin{array}{l}\text { Analytical } \\
\text { Limit }\end{array}$ & 3 & 400 & 100 & 30 & 200 & 2 \\
\hline \multicolumn{7}{|c|}{ Columbia Basin (Continued) } \\
\hline $3-9$ & - & 1400 & - & 37 & & \\
\hline $3-23$ & - & 950 & - & 33 & & \\
\hline $4-14$ & - & 1200 & - & $=$ & & 8.7 \\
\hline $4-27$ & - & 1300 & - & - & & \\
\hline $5-11$ & - & 1400 & - & - & & \\
\hline $5-25$ & - & 1500 & - & - & & \\
\hline $6-8$ & 4 & 1300 & - & 33 & & \\
\hline $6-22$ & - & 1500 & - & - & & \\
\hline $7-14$ & 4 & 1400 & - & - & & \\
\hline $7-28$ & - & 1400 & - & - & & \\
\hline $8-11$ & - & 1100 & - & - & & \\
\hline $8-25$ & - & 1300 & - & - & & \\
\hline $9-16$ & - & 1300 & - & - & & \\
\hline $9-29$ & - & 1300 & - & - & & \\
\hline $10-13$ & - & 1400 & - & - & & - \\
\hline $10-26$ & - & 1200 & - & - & & \\
\hline $11-10$ & 4 & 1600 & - & - & & \\
\hline $11-22$ & 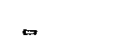 & 1400 & 200 & - & & \\
\hline $12-15$ & 4 & 11.00 & - & - & & \\
\hline $12-30$ & - & 1200 & - & - & & \\
\hline \multicolumn{7}{|c|}{ Benton City - Prosser } \\
\hline $1-13$ & 3 & 1200 & - & - & & \\
\hline $1-27$ & 3 & 1400 & - & - & & \\
\hline $2-10$ & - & 1300 & - & - & & \\
\hline $2-24$ & - & 1600 & - & 33 & & \\
\hline $3-9$ & - & 1600 & ـ & 30 & & \\
\hline $3-23$ & - & 1100 & $=$ & - & & \\
\hline $4-14$ & - & 1400 & - & - & & \\
\hline $4-27$ & - & 1300 & - & 36 & & \\
\hline $5-11$ & - & 1400 & . & - & & \\
\hline $5-25$ & - & 1200 & - & 36 & & \\
\hline 6.8 & - & 1100 & - & 30 & & 2.4 \\
\hline $6-22$ & 3 & 1300 & - & - & & \\
\hline $7-14$ & 3 & 1500 & - & - & & \\
\hline $7-28$ & - & 1400 & - & - & & \\
\hline
\end{tabular}

Results less than analytical limit are indicated by a $(-)$. No entry indicates no analysis made. 
APPENDIX F

TABLE 1 (Continued)

CONCENTRATIONS OF RADIONUCLIDES IN MILK - 1966 picocuries/1iter

\begin{tabular}{|c|c|c|c|c|c|c|}
\hline Date & ${ }^{13 I_{I}}$ & $40_{K}$ & ${ }^{65} \mathrm{zn}$ & $137 \mathrm{Cs}$ & $32_{p}$ & $90_{\mathrm{Sr}}$ \\
\hline $\begin{array}{l}\text { Analytical } \\
\text { Limit }\end{array}$ & 3 & 400 & 100 & 30 & 200 & 2 \\
\hline \multicolumn{7}{|c|}{ Benton City - Prosser (Continued) } \\
\hline $8-11$ & - & 1100 & - & - & & \\
\hline $8-25$ & - & 1200 & - & - & & \\
\hline $9-16$ & - & 1400 & - & - & & \\
\hline $9-29$ & - & 1300 & - & - & & \\
\hline $10-13$ & - & 1300 & - & - & & \\
\hline $10-26$ & - & 1300 & - & - & & \\
\hline $11-10$ & 4 & 1300 & - & - & & \\
\hline $11-22$ & - & & & & & \\
\hline $12-15$ & - & 1200 & 110 & - & & \\
\hline $12-30$ & - & 1300 & - & - & & \\
\hline
\end{tabular}

Local Purchase - Commercial Milk

\begin{tabular}{ll}
\hline Brand & A \\
$1-5$ & \\
$1-21$ & - \\
$2-3$ & - \\
$2-17$ & - \\
$3-16$ & - \\
$4-22$ & - \\
$5-5$ & - \\
$5-19$ & 3 \\
$6-15$ & - \\
$7-7$ & - \\
$7-21$ & - \\
$8-3$ & 3 \\
$8-18$ & - \\
$9-8$ & - \\
$9-22$ & - \\
$10-11$ & - \\
$10-20$ & - \\
$11-3$ & - \\
$11-17$ & - \\
$12-7$ & - \\
$12-20$ & -
\end{tabular}

Results less than analytical limit are indicated by a (-). No entry indicates no analysis made. 


\section{APPENDIX $\mathrm{F}$}

TABLE 1 (Continued)

\section{CONCENTRATIONS OF RADIONUCLIDES IN MILK - 1966} picocuries/liter

\begin{tabular}{|c|c|c|c|c|c|}
\hline Date & ${ }^{13} 1_{I}$ & $40_{K}$ & $65 \mathrm{zn}$ & $1.37 \mathrm{Cs}$ & $32_{P}$ \\
\hline Analytical & 3 & 400 & 100 & 30 & 200 \\
\hline
\end{tabular}

Limit

Local Purchase - Commercial Milk

Brand $\mathrm{F}$

$\overline{1}=\overline{5}-$

1600

54

$1=21$

$-$

$2-3$

$2 \times 17$

$=$

$3-16$

$4-7$

$4-22$

5-5

$5-19$

$6-15$

$7-7$

$7-21$

$8-3$

$8-18$

$9-8$

9-22

$10-11$.

10-20

$11 \mathrm{c} 3$

$11 \backsim 17$

$12 \mathrm{c} 7$

$12-20$

Brand $\mathrm{H}$

$\overline{1}-\overline{5}$

$1-2.1$

$2-3$

2-17

$3-16$

4 es 7

4-22

$5-5$

5-19

$\rightarrow$

$-$

4.3

$-$

-

$-$

-

$-$

3

$-$

-

$-$

$-$

$-$

$\infty$

$-$

$-$

$-$

-

Results less than analytical 1 imit are indicated by $(\rightarrow)$. No entry indicates no analysis made. 


\section{APPENDIX $F$ \\ TABLE 1 (Continued)}

CONCENTRATIONS OF RADIONUCLIDES IN MILK - 1966

picocuries/liter

\begin{tabular}{|c|c|c|c|c|c|c|}
\hline Date & ${ }^{13} I_{I}$ & $40_{K}$ & $65 \mathrm{zn}$ & $137 \mathrm{Cs}$ & $32 p$ & $90_{\mathrm{Sr}}$ \\
\hline $\begin{array}{l}\text { Analytical } \\
\text { Limit }\end{array}$ & 3 & 400 & 100 & 30 & 200 & 2 \\
\hline
\end{tabular}

\section{Brand $\underline{H}$}

$\frac{B}{6}-15=$

$7-7$

$7-21$

$8-3$

$8-18$

$9-8$

$9-22$

10-11

$10-20$

$11-3$

$11-17$

$12-7$

$12-20$

$\begin{array}{ll}- & 1600 \\ - & 1400 \\ - & 1300 \\ - & 1400 \\ - & 1400 \\ - & 1300 \\ - & 1500 \\ 3 & 1300 \\ - & 1400 \\ - & 1500 \\ 5 & 1600 \\ - & 1300 \\ - & 1200\end{array}$

$\begin{array}{ll}- & 79 \\ - & 66 \\ - & 79 \\ - & 56 \\ - & 49 \\ - & 40 \\ - & 42 \\ - & 67 \\ - & 42 \\ - & - \\ - & - \\ - & 33 \\ - & -\end{array}$

Results less than analytical limit are indicated by a (-). No entry indicates no analysis made. 


\section{APPENDIX F \\ TABLE 2 \\ CONCENTRATIONS OF RADIONUCLIDES IN \\ HAY AND PASTURE GRASS - 1966 \\ picocuries/gram}

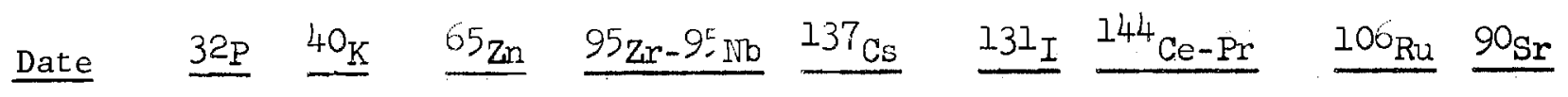

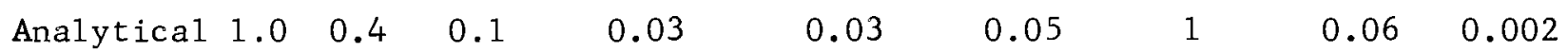

Limit

Riverview Irrigation District

\begin{tabular}{|c|c|c|c|c|c|c|c|c|c|}
\hline \multicolumn{2}{|l|}{$1-4$} & 8.8 & - & 0.49 & 0.25 & - & 2 & 2.2 & 0.131 \\
\hline \multicolumn{2}{|l|}{$4-26$} & 21 & & 0.18 & 0.50 & - & 2 & - & \\
\hline \multicolumn{2}{|l|}{$5-3$} & 23 & & 0.29 & 0.37 & - & - & 1.2 & \\
\hline \multicolumn{2}{|l|}{$5-10$} & 24 & & 0.53 & 0.44 & - & 4 & - & \\
\hline \multicolumn{2}{|l|}{$5-17$} & 5.8 & 1.1 & - & - & - & - & - & \\
\hline \multicolumn{2}{|l|}{$5-24$} & 6.4 & 0.51 & - & 0.07 & - & - & - & \\
\hline \multicolumn{2}{|l|}{$5-31$} & 5.2 & 0.21 & 1.1 & 0.05 & 0.27 & 6 & 2.5 & \\
\hline \multicolumn{2}{|l|}{$6-7$} & 6.0 & 0.28 & 0.28 & 0.12 & 0.05 & 1 & - & \\
\hline \multicolumn{2}{|l|}{$6-14$} & 5.6 & 0.30 & 0.62 & 0.05 & - & 2 & 0.96 & \\
\hline \multicolumn{2}{|l|}{$6-21$} & 23 & - & 1.9 & 0.25 & - & 1 & 0.60 & \\
\hline \multicolumn{2}{|l|}{$6-28$} & 5.0 & 0.48 & 0.18 & 0.05 & - & - & - & \\
\hline \multicolumn{2}{|l|}{$7-5$} & 7.5 & - & 1.2 & 0.19 & - & 3 & 2.0 & \\
\hline $7-12$ & 1.1 & 11 & - & 0.08 & 0.04 & 0.13 & - & & \\
\hline \multicolumn{2}{|l|}{$7-19$} & 21 & - & 0.45 & 0.05 & $=$ & - & - & \\
\hline \multicolumn{2}{|l|}{$7-26$} & 24 & 0.30 & 0.42 & 0.12 & - & - & - & \\
\hline \multicolumn{2}{|l|}{$8-4$} & 7.7 & 0.25 & 0.48 & 0.31 & 0.08 & 1 & 1.5 & \\
\hline \multirow{2}{*}{\multicolumn{2}{|c|}{$\begin{array}{l}8-9 \\
8-23\end{array}$}} & 10 & 3.3 & 6.8 & 0.80 & 0 & 7 & 1.9 & \\
\hline & & 13 & 0.82 & 0.76 & 0.24 & - & - & - & \\
\hline \multicolumn{2}{|l|}{$8-30$} & 11 & 2.2 & 0.94 & 0.33 & - & 4 & - & \\
\hline \multicolumn{2}{|l|}{$9-6$} & 7.9 & 5.4 & 0.19 & 0.11 & - & 2 & - & \\
\hline $9-13$ & - & 16 & 0.51 & 0.40 & 0.19 & - & - & - & \\
\hline \multicolumn{2}{|l|}{$9-20$} & 23 & 0.40 & 0.26 & 0.13 & 0.23 & - & - & \\
\hline \multicolumn{2}{|l|}{$9-27$} & 25 & - & 0.99 & 0.09 & - & - & - & \\
\hline \multicolumn{2}{|l|}{$10-4$} & 29 & 0.72 & 0.05 & 0.09 & - & - & - & \\
\hline $10-11$ & 1.1 & 29 & 0.96 & 0.10 & 0.13 & - & - & - & \\
\hline \multicolumn{2}{|l|}{$10-18$} & 28 & 0.38 & 0.03 & 0.11 & - & - & - & \\
\hline \multicolumn{2}{|l|}{$10-25$} & 26 & 0.53 & 0.10 & 0.04 & 0.07 & - & 0.81 & \\
\hline \multicolumn{2}{|l|}{$11-1$} & 24 & 0.56 & 0.09 & 0.11 & - & - & 0.60 & \\
\hline $11-8$ & - & 24 & 0.24 & 0.03 & - & - & 3 & - & \\
\hline & 3.6 & 0.12 & 0.08 & - & 0.58 & - & - & \\
\hline $11-22$ & & 20 & - & 0.04 & $=$ & - & - & 1.6 & \\
\hline $11-29$ & & 6.6 & 0.18 & 0.07 & 0.07 & - & - & 0.81 & \\
\hline $12-6$ & & 23 & 0.30 & 0.08 & 0.04 & - & - & - & \\
\hline $12-13$ & & 22 & - & - & 0.03 & - & - & 1.3 & \\
\hline $12-20$ & & 17 & 0.16 & 0.04 & $=$ & - & - & - & \\
\hline $12-27$ & & 21 & 0.12 & - & - & - & - & - & \\
\hline
\end{tabular}

Results less than reporting 1 imit are indicated by a (-). No entry indicates no analysis made. 
APPENDIX F

TABLE 2 (Continued)

CONCENTRATIONS OF RADIONUCLIDES IN

HAY AND PASTURE GRASS - 1966

picocuries/gram

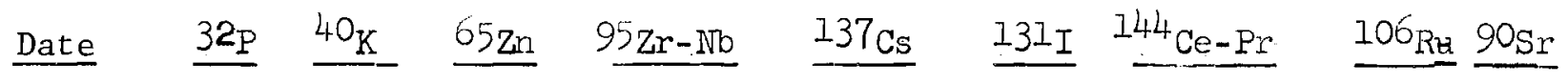

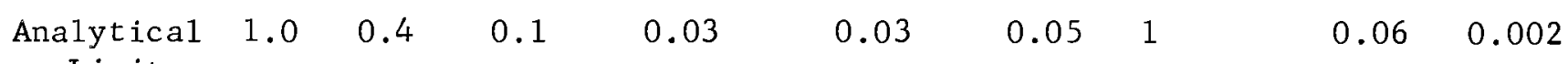

Limit

$\frac{\text { Ringold Farm No. } 1}{5-26}$

$5-3$

$5-10$

$5-17$

$5-24$

$5-31$

$6-7$

$6-14$

$6-21$

$6-28$

$7-5$

$7-12$

$7-19$

$7-26$

$8-3$

$8-9$

$8-16$

$8-23$

$8-30$

9-6

$9-13$

9-20

9-27

10-4

5.3

0.27

5.3

$-$

0.27

5.1

0.24

5.0

0.13

6.6

0.29

6.4

0.22

6.4

0.88

7.7

0.22

5.4

0.26

5.6

0.10

5.5

0.71

0.44

2.4

10

10

1.0

9.7

0.96

9.1

1.2

9.1

0.93

15

13

$50 \quad 11$

$10-11$

5.2

5.0

5.1

1.3

$-$

$-$

$-$

0.36

1.7

0.89

0.73

1.3

0.55

0.09

0.14

0.56

2.2

0.45

0.14

0.26

1.6

0.99

0.31

0.67

$-$

$-$

0.44

0.11

$10-18$

5.3
4.7

1.5

-

0.03

0.21

$\overline{0.05}$

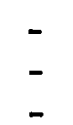

0.03

0.03

- 0.06

0.04

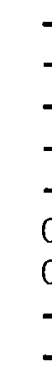

$-$

-

$-$

0.17

0.20

0.22

0.12

0.09

0.07

-

0.10

0.19

0.06

$-$

0.0

0.04

-

- 3

$0.16 \quad 6$

0.06

4
2

\section{-}

Ringold Farm No. 2

\begin{tabular}{ll}
\hline $9-13$ & 29 \\
$10-25$ & 17 \\
$10-27$ & 15 \\
$11-1$ & 26 \\
$11-3$ & 28 \\
$11-8$ & 30 \\
$11-10$ & 37 \\
$11-15$ & 22 \\
$11-22$ & 14
\end{tabular}

$\begin{array}{lllllll}0 & & 0.20 & 0.21 & - & 1.5 & . \\ 0.30 & 0.12 & 0.16 & - & - & - & . \\ 0.27 & 0.09 & 0.05 & - & - & - & \vdots \\ 0.71 & 0.03 & 0.08 & - & - & - & - \\ 0.54 & 0.06 & 0.06 & - & - & - & - \\ 0.35 & - & - & - & - & -60 & \therefore \\ 0.45 & 0.14 & 0.07 & - & - & - & \\ 0.22 & 0.10 & -.0 .07 & 0.07 & - & 0.67 & \\ 0.56 & 0.16 & 0.09 & 0.12 & - & - & \end{array}$

Resu1ts less than reporting limit are indicated by a (-). No entry indicates no analys is made. 
APPENDIX F

TABLE 2 (Continued)

CONCENTRATIONS OF RADIONUCLIDES IN

HAY AND PASTURE GRASS - 1966

picocuries/gram

Date $\quad 32_{\mathrm{P}} \quad 40_{\mathrm{K}} \quad 65_{\mathrm{Zn}}-95_{\mathrm{Zr}-\mathrm{Nb}}^{137 \mathrm{Cs}} 131_{\mathrm{I}} 144 \mathrm{Ce}-\mathrm{Pr} \quad 106_{\mathrm{Ru}} 90_{\mathrm{Sr}}$

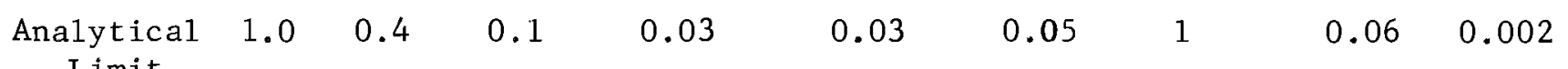

Limit

Ringold Farm No. 2 (Continued)

$\begin{array}{llll} & & & \\ 11-29 & 22 & 0.52 & 0.10 \\ 12-6 & 29 & - & - \\ 12-13 & 25 & 0.12 & - \\ 12-20 & 25 & 0.50 & 0.07 \\ 12-27 & 30 & 1.1 & -\end{array}$

$\frac{\text { Pasco Farm No. } 1}{1-5}$

$\begin{array}{lll}5-10 & 9.0 & 6.7\end{array}$

$5-16 \quad 5.8$

$5-17 \quad 5.3$

$5-24 \quad 4.8$

$5-31 \quad 5.8$

$\begin{array}{lll}6-7 & - & 5.8\end{array}$

$6-14 \quad 6.7$

$6-21 \quad 5.1$

6-28 4.9

$7-5 \quad 5.5$

$7-13-4.7$

$\begin{array}{ll}7-27 & 7.8\end{array}$

$8-3 \quad 4.6$

$8-9=4.2$

\&-16 $\quad 5.3$

8-23 6.7

8-30 6.1

$9-6$

$9-13$

$9-20$

9-27

10-4

$10-11$

$10-18$

$10-24$

$10-25$

$10-27$

10-31

$$
2.3
$$

6.9

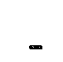

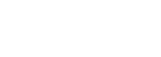

0.10

-
-
-
0.10
1.7

2.1

0.30

0.44

0.11

ค. 20

0.21

0.34

0.10

0.04

0.03

0.25

5.6

0.05

$$
\begin{aligned}
& 0.11 \\
& 0.03 \\
& - \\
& 0.05
\end{aligned}
$$

11-1

11-3

11-7

$$
6.0
$$

6.3

5.4

3.2

0.28

0.03

0.03
0.03

0.04

0.03

0.03

0.04

21

16

27

24

23

24

11-8

24

0.05

0.04

$-$

0.70

0.17

$$
\text { - }
$$

0.03

0.15

0.38
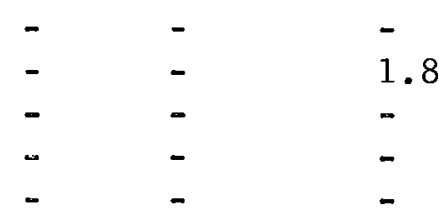

Results less than reporting limit are indicated by (-). No entry indicates no analysis made. 


\section{APPENDIX F}

TABLE 2 (Continued)

\section{CONCENTRATIONS OF RADIONUCLIDES IN} HAY AND PASTURE GRASS - 1966

$$
\mathrm{p} \text { icocuries/gram }
$$

Date $\quad \underline{32_{\mathrm{F}}} \quad 4 \mathrm{O}_{\mathrm{K}} \quad \underline{65 \mathrm{Zn}} \quad \underline{95 \mathrm{zr}-\mathrm{Nb}} \quad \underline{137 \mathrm{Cs}} \quad \underline{13 I_{\mathrm{I}}} 95 \mathrm{Ce}-\mathrm{Pr} \ldots 106_{\mathrm{Ru}} \underline{90_{\mathrm{Sr}}}$

$$
\begin{array}{llllllllll}
\text { Analytical } & 1.0 & 0.4 & 0.1 & 0.03 & 0.03 & 0.05 & 1 & 0.06 & 0.002
\end{array}
$$

Limit

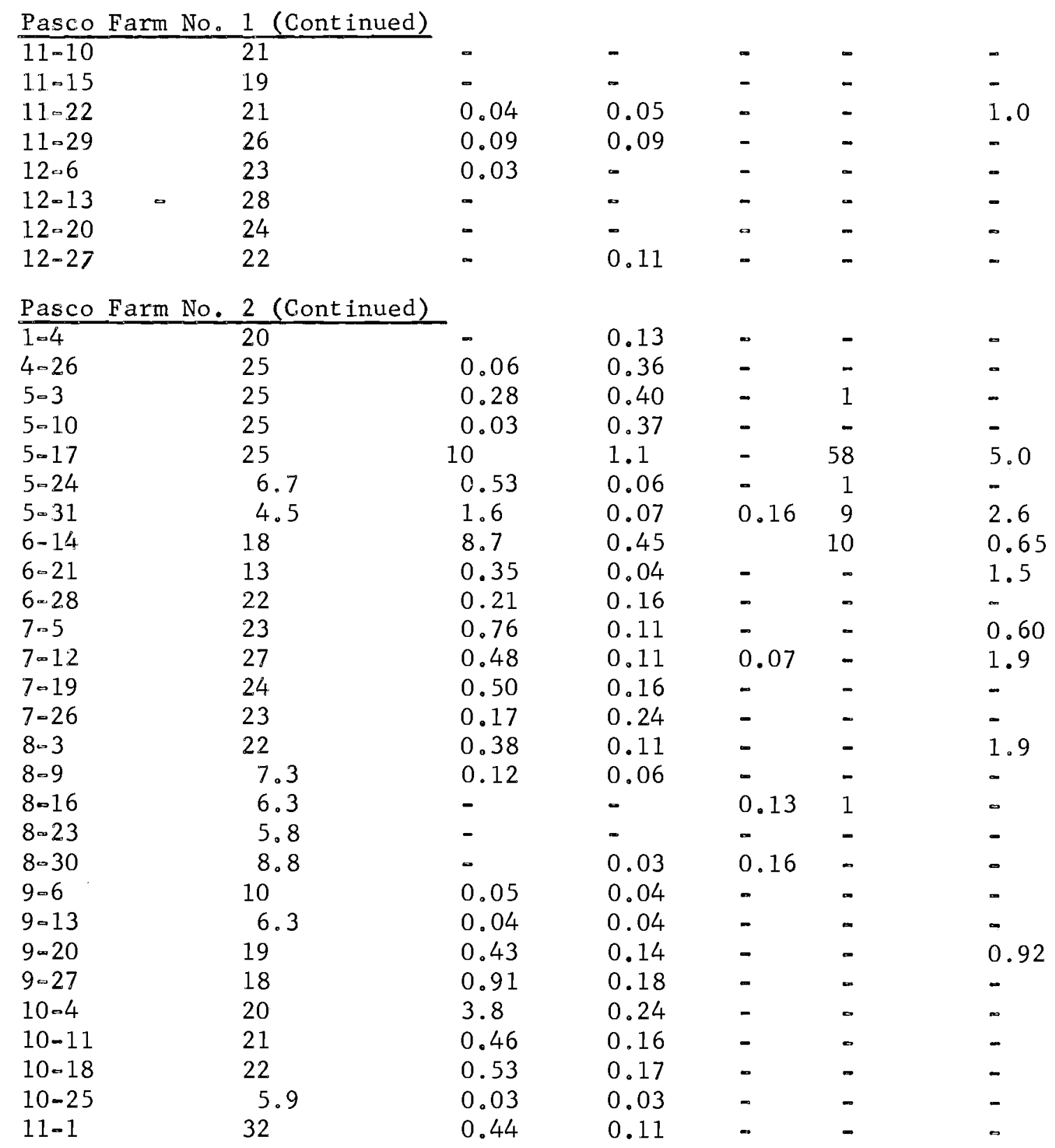

Results less than reporting limit are indicated by (-). No entry indicates no analysis made. 


\begin{tabular}{|c|c|c|c|c|c|c|c|c|c|}
\hline \multicolumn{10}{|c|}{$\begin{array}{c}\text { APPENDIX F } \\
\text { TABLE } 2 \text { (Continued) }\end{array}$} \\
\hline \multirow[b]{3}{*}{ Date } & \multirow[b]{3}{*}{$32 \mathrm{p}$} & \multicolumn{4}{|c|}{$\begin{array}{l}\text { CONCENTRATIONS OF RADIONUCLIDES } \\
\text { HAY AND PASTURE GRASS - } 1966 \\
\end{array}$} & IN & \multirow[b]{3}{*}{$144 \mathrm{Ce}-\mathrm{Pr}$} & \multirow[b]{3}{*}{$106_{\mathrm{Ru}}$} & \multirow[b]{3}{*}{$90_{\mathrm{Sr}}$} \\
\hline & & \multirow[b]{2}{*}{$4 \mathrm{O}_{\mathrm{K}}$} & \multicolumn{3}{|c|}{ picocuries/gram } & \multirow[b]{2}{*}{${ }^{13} I_{I}$} & & & \\
\hline & & & $65 \mathrm{zn}$ & $95 \mathrm{Zr}-\mathrm{Nb}$ & $137 \mathrm{Cs}$ & & & & \\
\hline $\begin{array}{l}\text { Analytical } \\
\text { Limit }\end{array}$ & 1.0 & 0.4 & 0.1 & 0.03 & 0.03 & 0.05 & 1 & 0.06 & 0.002 \\
\hline \multicolumn{4}{|c|}{ Pasco Farm No. 2 (Continued) } & & & & & & \\
\hline \multicolumn{2}{|l|}{$11-8$} & \multicolumn{2}{|l|}{31} & 0.15 & 0.04 & - & - & - & \\
\hline $11-15$ & & \multicolumn{2}{|l|}{18} & 0.21 & 0.10 & - & - & - & \\
\hline $11-22$ & & \multicolumn{2}{|l|}{26} & 0.16 & 0.12 & - & - & 0.60 & \\
\hline $11-29$ & & \multicolumn{2}{|l|}{21} & 0.09 & 0.09 & - & - & - & \\
\hline $12-6$ & & \multicolumn{2}{|l|}{26} & 0.04 & 0.15 & - & - & - & \\
\hline $12-13$ & & \multicolumn{2}{|l|}{14} & - & 0.07 & - & - & - & \\
\hline $12-20$ & & \multicolumn{2}{|l|}{22} & 0.18 & 0.18 & - & - & - & \\
\hline $12-27$ & & \multicolumn{2}{|l|}{12} & $=$ & 0.05 & - & - & - & \\
\hline \multicolumn{10}{|l|}{ Eltopia } \\
\hline$\overline{1-4}$ & & \multicolumn{2}{|l|}{8.5} & 0.39 & 0.29 & - & 1 & - & \\
\hline $4-26$ & & 5.0 & & - & - & - & - & - & \\
\hline $5-4$ & & 6.0 & & - & - & - & - & - & \\
\hline $5-10$ & & 5.4 & & - & 0.03 & - & - & - & \\
\hline $5-18$ & & 18 & & - & 0.12 & - & - & - & \\
\hline $5-24$ & & 24 & & - & 0.09 & - & - & $\infty$ & \\
\hline $6-1$ & & 23 & & 0.03 & 0.08 & - & - & - & \\
\hline $6-14$ & & 25 & & 0.87 & 0.13 & - & 1 & 1.7 & \\
\hline $6-21$ & & 20 & & 1.4 & 0.15 & - & - & 1.4 & \\
\hline $6-28$ & & 19 & & 2.9 & 0.30 & - & 3 & 2.5 & \\
\hline $7-5$ & & 24 & & 0.44 & 0.07 & 0.06 & - & 1.5 & \\
\hline $7-12$ & & 6.8 & & 0.11 & - & - & - & 0.60 & \\
\hline $7-19$ & & 24 & & 0.76 & 0.20 & - & - & - & \\
\hline $7-26$ & & 25 & & 0.70 & 0.27 & - & - & - & \\
\hline $8-4$ & & 31 & & 0.22 & 0.15 & - & - & 1.9 & \\
\hline $8-9$ & & 24 & & 0.16 & 0.08 & - & - & - & \\
\hline $8-16$ & & 25 & & - & 0.05 & - & - & - & \\
\hline $8-23$ & & 24 & & - & - & - & - & - & \\
\hline $8-30$ & & 26 & & 0.04 & 0.04 & - & 2 & - & \\
\hline $9-6$ & & 20 & & - & - & - & 8 & - & \\
\hline $9-13$ & & 30 & & - & 0.05 & 0.08 & - & - & \\
\hline $9-20$ & & 33 & 0.35 & - & 0.13 & 0.06 & - & - & \\
\hline $9-27$ & & 28 & & 0.03 & 0.04 & 0.31 & - & - & \\
\hline $10-4$ & & 24 & & 0.48 & 0.11 & - & - & - & \\
\hline $10-18$ & & 28 & & - & 0.06 & - & - & - & \\
\hline $10-25$ & & 18 & & - & 0.04 & - & - & - & \\
\hline $11-1$ & & 25 & 0.33 & 0.03 & 0.04 & - & - & - & - \\
\hline $11-8$ & & 28 & & - & 0.07 & 0.11 & - & 0.78 & \\
\hline
\end{tabular}

Results less than reporting limit are indicated by (-). No entry indicates no analys is made. 
APFENDIX F

TABIE 2 (Continued)

CONCENTRATIONS OF RADIONUCLIDES IN

HAY AND FASTURE GRASS - 1966

$$
\text { picocuries/gram }
$$

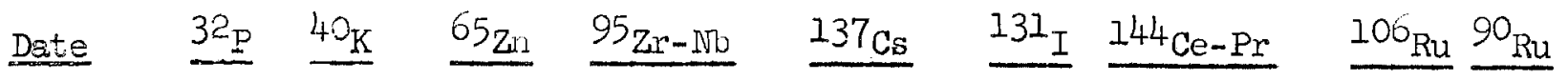
Analytical 1.0
0.4
0.1
0.03
$0.03 \quad 0.05$
1
$0.06 \quad 0.002$

Limit

Eltopia (Continued)

\begin{tabular}{|c|c|}
\hline $\begin{array}{l}11 \times 17 \\
11 \times 20 \\
11-29 \\
12=6 \\
22-13 \\
12=00 \\
22-27\end{array}$ & $\begin{array}{l}37 \\
21 \\
25 \\
26 \\
18 \\
14 \\
20\end{array}$ \\
\hline Richland & Farm No \\
\hline $\begin{array}{l}=6 \\
6-23 \\
6-30 \\
7-7 \\
7-28 \\
8-28 \\
8-21 \\
8.8\end{array}$ & $\begin{array}{r}1.5 \\
4.4 \\
6.8 \\
6.2 \\
7.6 \\
9.7 \\
7.8 \\
7.2\end{array}$ \\
\hline
\end{tabular}

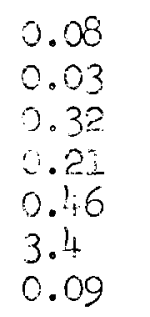

$$
\begin{aligned}
& 0.14 \\
& 0.05 \\
& 0.31 \\
& 0.23 \\
& 0.12 \\
& 0.19 \\
& 0.21
\end{aligned}
$$

0.47

0.20

0.42

0.53

0.06

5.2

0.66

0.44

$\begin{array}{ll}0.10 & \\ - & \\ - & \\ - & \end{array}$

$\begin{array}{ll}= & 3.7 \\ = & = \\ = & = \\ = & = \\ = & =\end{array}$

West Richiand, Farm No.?

$10=2$ ?

$11-3$

$11-10$

$12-17$

$11-23$

$12-1$

12.8

$12=15$

$12 \cdot 22$

$22-29$

Benton dity

i=6

$4-28$

505

$5=1 \hat{c}$

$5-29$

$5-26$

25

21

11

10

22

23

20

24

6.3
30
21
15
6.8
7.6

$\infty$

0.23

0.26

0.29

0.23

0.14

0.04

0.05

0.31

0.07

$-$

-

0.04
0.30

0.03

0.11

0.07

0.06

0.33

0.18

0.17

$=$
$=$
$=$
$=$
0.10

$\begin{array}{ll}1 & - \\ - & = \\ - & 1.0 \\ 1 & 0.74 \\ 1 & = \\ 1 & = \\ 1 & - \\ 2 & -\end{array}$

0.19

0.19

0.19

0.30

0.27

0.05

0.09

0.16

0.29

0.40

0.74

-

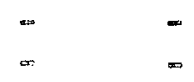

$-\quad 0.60$

$=0.50$

-

-

$=$

-

1

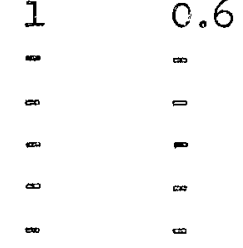




\begin{tabular}{|c|c|c|c|c|c|c|c|c|c|}
\hline & & & & $\begin{array}{l}\text { AFPEND } \\
\text { SIE } 2(\mathrm{Co}\end{array}$ & $\begin{array}{l}F \\
\text { irued) }\end{array}$ & & & & \\
\hline & & & $\begin{array}{l}\text { CENTRA } \\
\text { HAY AN }\end{array}$ & $\begin{array}{l}\text { LNS OF } \\
\text { PASTURE }\end{array}$ & $\begin{array}{l}\text { IONUCLI } \\
\text { ASS }-1\end{array}$ & 5 & & & \\
\hline & & & & oicocurie & gram & & & & \\
\hline & 32 & 40 & 65 & 95 & 237 & 131 & 144 & 106 & 90 \\
\hline Dete & $P$ & $\mathrm{~K}$ & $\underline{Z n}$ & $\mathrm{Z}=\mathrm{Nb}$ & $\mathrm{CS}_{\mathrm{E}}$ & & $\mathrm{Ce}-\mathrm{Pr}$ & Ru & $\mathrm{Sr}$ \\
\hline $\begin{array}{c}\text { Analytical } \\
\text { Limit }\end{array}$ & 1.0 & 0.4 & 0.1 & 0.03 & 0.03 & 0.05 & 1 & 0.06 & 0.002 \\
\hline Benton $C$ & ity & Contin & & & & & & & \\
\hline $6-2$ & & 6.2 & & 5.7 & 0.06 & - & $\infty$ & $\infty$ & \\
\hline $6-9$ & & 6.0 & & 0.38 & 0.06 & in & 2 & 0.91 & \\
\hline $6=16$ & & 7.0 & & 0.05 & $\infty$ & $\infty$ & $=$ & $\omega$ & \\
\hline $5-23$ & & 6.5 & & 0.30 & 0.04 & - & 1 & 0.60 & \\
\hline $6-30$ & & 8.9 & & 0.04 & $\Leftrightarrow$ & $\infty$ & $\infty$ & $m$ & \\
\hline $7-7$ & & 8.0 & & 0.37 & 0.04 & - & $\omega$ & - & \\
\hline $7-22$ & & 9.0 & & 0.39 & 0.12 & $\infty$ & $=$ & - & \\
\hline $7=28$ & & 8.0 & & 0.10 & 0.07 & $=$ & $=$ & - & \\
\hline $8-5$ & & 20 & & 3.0 & 0.27 & - & $=$ & 0.60 & \\
\hline 8.11 & & 19 & & 0.68 & 0.31 & - & $\infty$ & $\infty$ & \\
\hline $8-18$ & & 20 & & $1 . .3$ & 0.24 & - & $\therefore$ & - & \\
\hline 8.25 & & 28 & & 4.5 & 0.18 & - & - & $\Rightarrow$ & \\
\hline $9-1$ & & 19 & & 0.72 & 0.75 & - & 3 & 1.1 & \\
\hline $9 . \overline{8}$ & & 18 & & 0.91 & 0.24 & $\infty$ & - & $\infty$ & \\
\hline 9.15 & & 18 & & 1.1 & 0.25 & $\infty$ & $\infty$ & 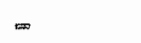 & \\
\hline $9=22$ & & 20 & 0.61 & 1.8 & 0.28 & - & - & - & \\
\hline $9=00$ & & 21 & & 0.22 & 0.24 & - & - & $\infty$ & \\
\hline$I 0 \mathrm{cc}$ & & 21 & & 2.3 & 0.29 & $\infty$ & - & $=$ & \\
\hline $23=13$ & & 25 & & 0.28 & 0.14 & - & $\infty$ & $\infty$ & \\
\hline 10.20 & & 18 & & 0.48 & 0.26 & - & 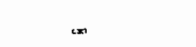 & $\infty$ & \\
\hline 50027 & & 38 & & 0.53 & $0.2^{2}$ & - & $\infty$ & 0.60 & \\
\hline$J i=3$ & & 22 & & 0.53 & 0.21 & - & - & $\approx$ & \\
\hline 11010 & & 19 & & 0.06 & 0.37 & 0.10 & - & - & \\
\hline $11=17$ & & 9.8 & & 0.93 & 0.18 & $=$ & $\infty$ & 0.60 & \\
\hline $12=23$ & & 53 & & 0.94 & 0.49 & $=$ & - & $\therefore$ & \\
\hline $12=1$ & & 16 & & 0.90 & 0.28 & 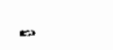 & $\infty$ & $\infty$ & \\
\hline 120.8 & & 20 & & 0.26 & 0.32 & 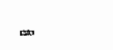 & ـ & $\Rightarrow$ & \\
\hline $12-15$ & & 21 & & 0.47 & 0.30 & - & $=$ & - & \\
\hline $12 \times 22$ & & 23 & & 0.18 & 0.16 & 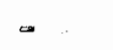 & - & $\infty$ & \\
\hline $12-69$ & & 17 & & 0.20 & 0.29 & $=$ & $=$ & $\infty$ & \\
\hline
\end{tabular}




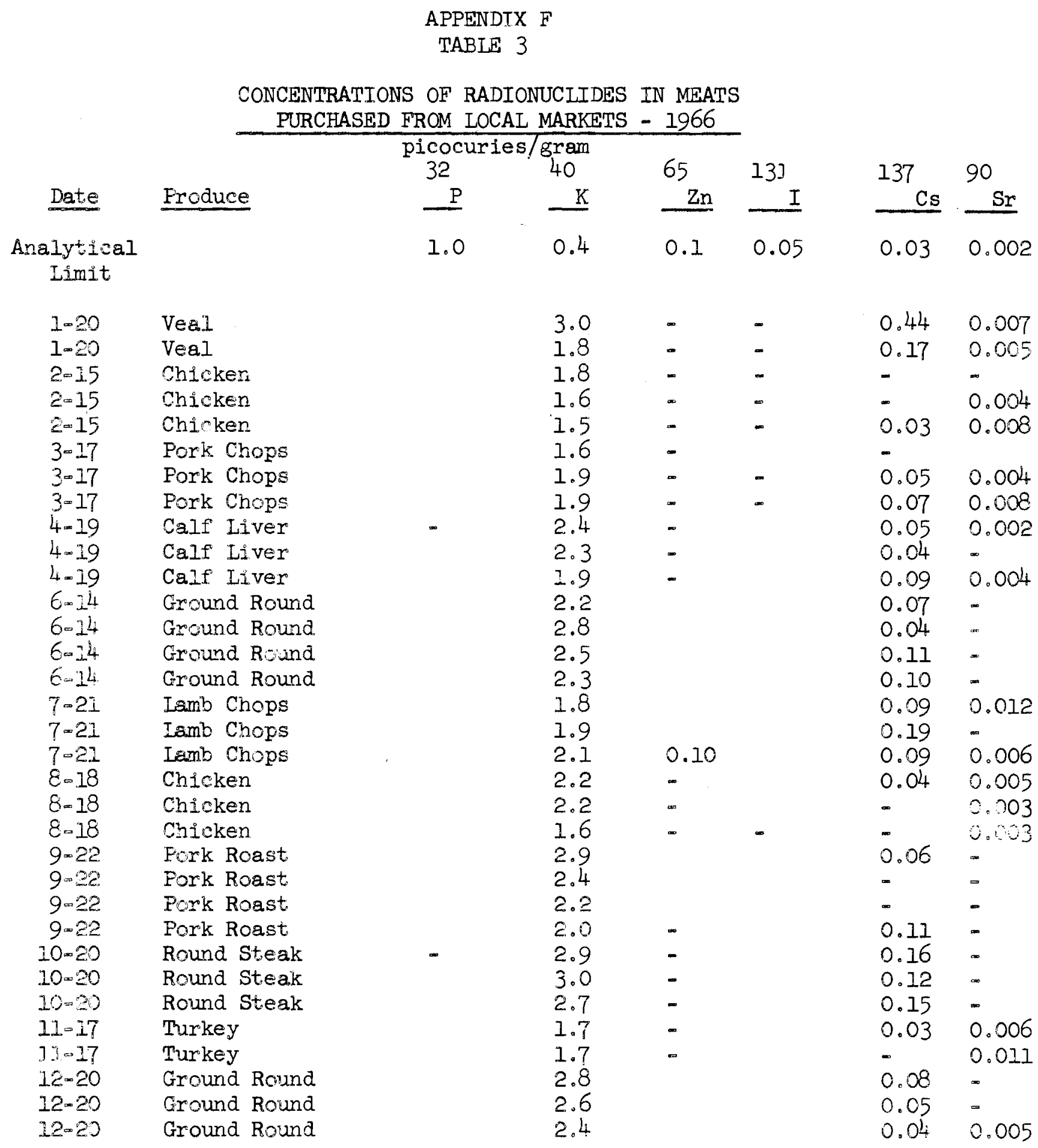

Results less than analytical limit are indicated by a (-). No entry indicates no analysis made. 


\section{APFENDIX E \\ TABIE 4}

\section{CONCENTRAIIONS OF RADIONUCIIDES IN \\ IOCALIY GROWN FARM PRODUCTS - 1966}

Ficocuries/gram

\begin{tabular}{|c|c|c|c|c|c|c|c|c|c|}
\hline Date & Produce & ${ }^{32} \mathrm{P}$ & $4 \mathrm{O}_{\mathrm{K}}$ & $65 \mathrm{Zn}$ & $\begin{array}{l}9^{5} \mathrm{Zx}- \\
95 \mathrm{Nb} \\
\end{array}$ & $13.1 \mathrm{I}$ & $137 \mathrm{Cs}$ & $\begin{array}{l}144 \mathrm{Ce} \\
144 \mathrm{Pr} \\
\end{array}$ & $90_{\mathrm{Sr}}$ \\
\hline $\begin{array}{r}\text { Analytical } \\
\text { Iimit }\end{array}$ & & 1.0 & 0.4 & 0.1 & 0.03 & 0.05 & 0.03 & 1.0 & 0.092 \\
\hline $4-22$ & Asparagus & & 2.3 & $\infty$ & & & $\Rightarrow$ & & $\therefore$ \\
\hline $4-22$ & Asparagus & & 2.7 & & & & $=$ & & 0.092 \\
\hline $4-27$ & Asparagus & & 2.5 & & & & - & & $=$ \\
\hline $6-16$ & Cherries & & 1.8 & & 0.04 & & - & & 0.06 \\
\hline 6017 & Cherries & & 2.2 & & & & & & - \\
\hline$E=17$ & Cherries & & 3.0 & & & & & & 0.002 \\
\hline $6=17$ & cherries & 3.8 & $\infty$ & 0.10 & & & 0.04 & & 0.027 \\
\hline $6=21$ & Eggs & 4.5 & 1.1 & 3.8 & & & $\infty$ & & $0.00 \%$ \\
\hline $6-21$ & Chioken & - & 2.8 & 2.7 & & $\infty$ & $\infty$ & & $\infty$ \\
\hline 6.28 & Cinjoken & & 1.9 & & & & 0.07 & & 0.003 \\
\hline $6-38$ & Eggs & $\infty$ & 1.3 & & & & - & & 0.002 \\
\hline $6=30$ & Rhubarb & 3.4 & 4.5 & $\infty$ & & & 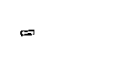 & & 0.030 \\
\hline $6-30$ & Ieaf Letrice & 1.7 & 4.3 & 0.39 & 0.07 & & - & & 0.032 \\
\hline $6-30$ & Radishes & $=$ & 3.5 & 0.13 & & & 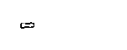 & & 0.023 \\
\hline 6.30 & $F \in a s$ & 2.3 & 2.0 & 0.24 & & & $\infty$ & & 0.056 \\
\hline $5 \times 30$ & Caynots & 1.3 & 3.1 & - & & & - & & 0.026 \\
\hline $6-30$ & Potatoes & $\Leftrightarrow$ & 3.8 & - & & & $\infty$ & & $=$ \\
\hline $6=30$ & REubario & & 4.0 & & & & - & & 0.010 \\
\hline 5030 & Potatoes & & 3.9 & & & & $\infty$ & & $=$ \\
\hline $6=30$ & Agricots & & 2.7 & & & & $=$ & & - \\
\hline $7-1$ & Lettuce & & & & & & & & \\
\hline $7 \cdot 1$ & Iettues & & 6.2 & & 0.29 & & 0.08 & $\omega$ & \\
\hline $7-1$ & Lettuce & & 3.2 & & 0.12 & & $\infty$ & - & \\
\hline $8-3$ & Cucumbers & & 1.8 & 0.30 & & & $=$ & & 0.020 \\
\hline $8-3$ & Corn on $\mathrm{Cob}$ & & 2.8 & 0.11 & & & $=$ & & $\infty$ \\
\hline $8=3$ & Tomatoes & & 1.5 & $\infty$ & & & 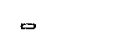 & & 0.004 \\
\hline $8-3$ & Green Apples & & 1.3 & - & & & $\infty$ & & $=$ \\
\hline 8.4 & Cabbage & & 2.9 & 0.10 & & & $\infty$ & & 0.004 \\
\hline $8=16$ & Cucumbers & $\infty$ & 1.5 & $\infty$ & & & $=$ & & $=$ \\
\hline $8-16$ & Corn on Cob & & 2.6 & $=$ & & & $=$ & & 0.008 \\
\hline 8.16 & Tomatces & $=r$ & 2.2 & $\infty$ & & & $\infty$ & & $=$ \\
\hline
\end{tabular}

Results less han analytical limit are indicated by a $(0)$. No entry Indicates no analysis made. 


\section{AFPENDIX F \\ TABIE 4 (Continued)}

CONCENTRATIONS OF RADIONUCL DES IN

LOCALIY GROWN FARM PRODUCTS - 1966

Picocuries/gram

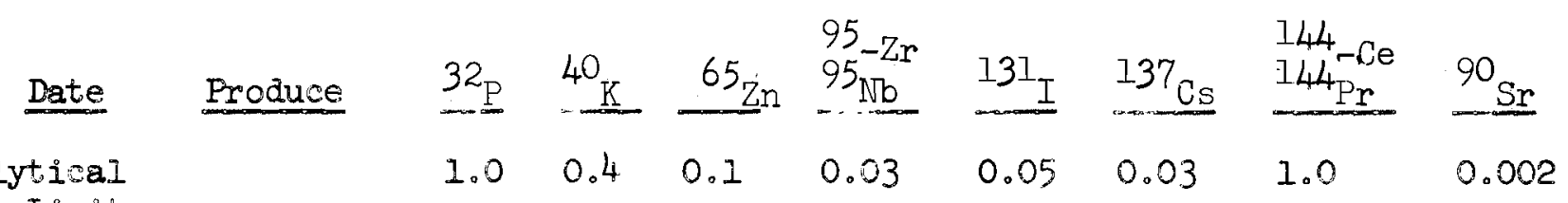

8.18 Feaches 2.3

8-18 Com on $\mathrm{Cob} \quad 2.9$

8.18 Feaches I.8

$8-18$ Peaches 1.9

Q.18 Cucumbers 1.5

$8 \times 18$ Peaches 2.0

8-18 Lettuce 4.3

8-18 Iettuce 2.8

$8-23$ Corn - 1.8

$8 \times 23$ Cucumbers - 1.6

8.23 Tcmatoes, 1.9

$8-23$ Feaches

8.23 Tomatoes

$9=5$ Tomatoes

905 Corn on $\mathrm{Cob}$

- 1.8 .

$\infty \quad 1.9=$

$1.9=$
$2.4=$

$2.6=$

-2.0 .

9-5 Cueumbers

905 Peaches

9.5 Apples

9.6 Tomatoes

- 2.2 .

-1.5 .

0.003

$\infty$

0.004

$-$

0.003

$\infty$

0.002

0.008

$-$

0.002

0.003

$2.6=$ 


\section{AFPENDIX F \\ TABIE 5}

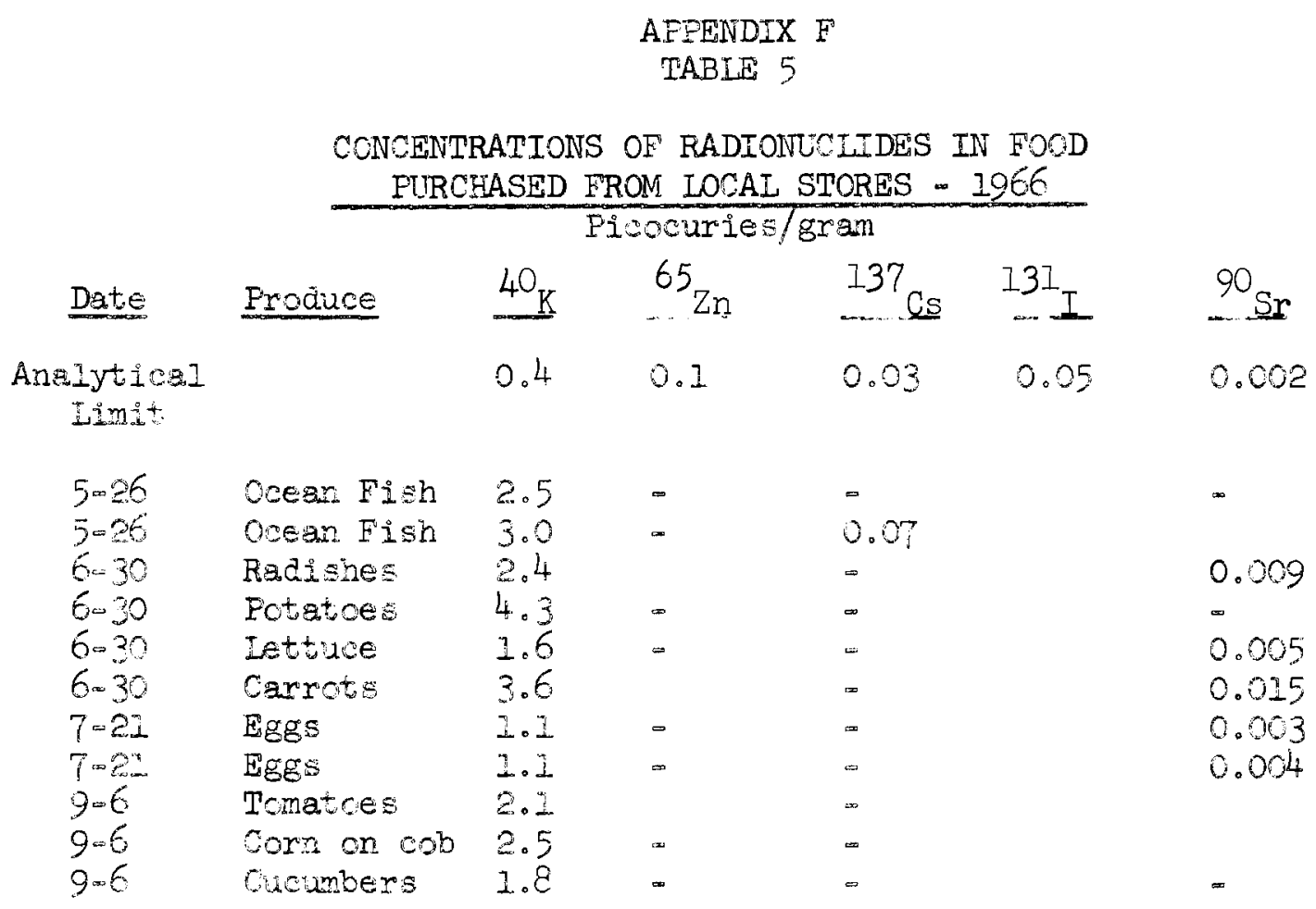

Results $2 e s s$ than analytical limit are indicated by $a(-)$. No entry irdicates no analysis made. 
BNWL-439 APP

APPENDIX G
131 I IN CATTLE THYROIDS 


\section{APFENDIX, G \\ TABLE 1}

\section{I I IN BEEF THYROIDS FRCM CAITIIIE \\ SLAEGHTERED AT SEVERAL IDCATIONS - 1966}

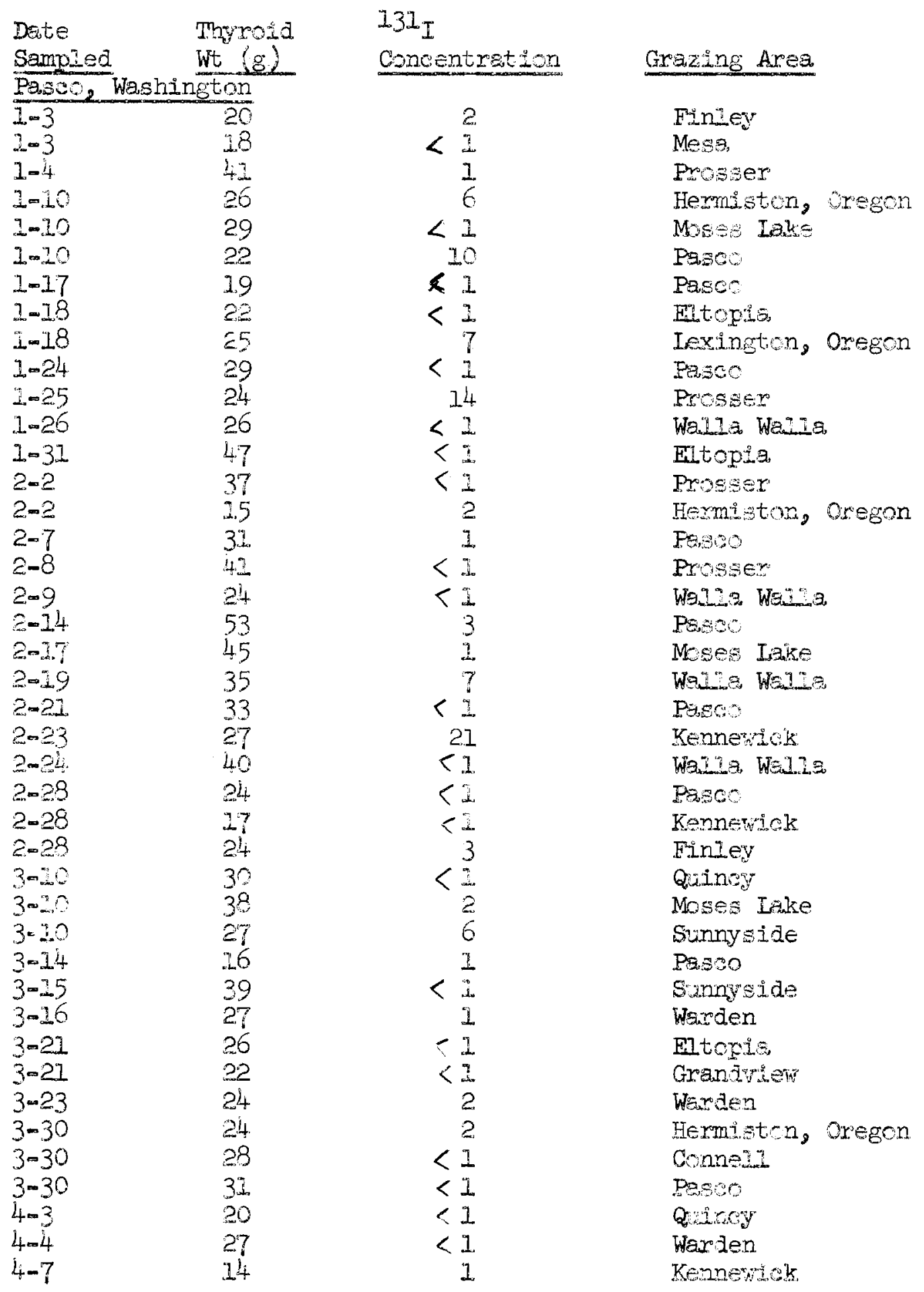


APPEIDIX G

TABLE 1 (Conuinued)

\begin{tabular}{|c|c|c|c|}
\hline \multicolumn{4}{|c|}{ The roid $\begin{array}{c}\text { Picocuries/tan } \\
\text { 3. }\end{array}$} \\
\hline Sarpled & Wt $(5)$ & Concen rauion & Grazin Ares \\
\hline Pasco, 1 & noton $(\mathrm{c}$ & ued) & \\
\hline $4-8$ & $1 \overline{9}$ & $<1$ & Prosser \\
\hline $4-11$ & 38 & 2 & Heri.is on, Oregon \\
\hline $4-12$ & 18 & $<1$ & Comell \\
\hline $4-18$ & 17 & $<1$ & Moses Lake \\
\hline $4-19$ & 10 & $<1$ & Pasco \\
\hline $4-2 I$ & 29 & $<1$ & Erosser \\
\hline $4-25$ & 17 & $<1$ & El opia \\
\hline $4-26$ & .15 & $<1$ & Warden \\
\hline $4-27$ & 20 & $<1$ & WaIla Walla \\
\hline $5-3$ & 30 & 3 & Pasco \\
\hline $5-5$ & 14 & $<1$ & Burbaik \\
\hline $5-5$ & 28 & 2 & Hermistor, Oresor \\
\hline $5-9$ & 33 & $<1$ & Warden \\
\hline $5-9$ & 15 & 13 & Plymouch \\
\hline 59 & 30 & $<1$ & Quiney \\
\hline $5-10$ & 17 & $<1$ & Warden \\
\hline $5-16$ & 14 & $<1$ & Kahlo us \\
\hline $5-18$ & 32 & 4 & Hermisuon, Oregon \\
\hline 524 & 22 & $<1$ & Prosser \\
\hline $5 \cdot 24$ & 33 & $<1$ & Mesa \\
\hline $5-24$ & 12 & $<1$ & Burbank \\
\hline $6-3$ & 22 & $<1$ & El bopia \\
\hline $0-3$ & 24 & $<1$ & Burbank \\
\hline $0-3$ & 24 & $<1$ & Kanlobus \\
\hline $0-8$ & 17 & 75 & Prosser \\
\hline $0-8$ & 11 & $<1$ & Pasco \\
\hline $0-8$ & 24 & $<1$ & Quincy \\
\hline $5-10$ & 3.1 & 32 & Walla Walla \\
\hline $0-13$ & 31 & 33 & Hermisco, oregon \\
\hline 6.13 & .17 & $<1$ & Warden \\
\hline $0-20$ & 18 & 3 & Paseo \\
\hline $0-21$ & 35 & 10 & Prosser \\
\hline $6-22$ & 16 & $<?$ & Bl opia \\
\hline $6-24$ & 38 & $?$ & Prosser \\
\hline $6-27$ & 20 & 2 & Wesi Richland \\
\hline $0-28$ & 24 & 35 & Hermisuon, orecon \\
\hline $7-5$ & 20 & $<1$ & Warden \\
\hline $7-7$ & 34 & 9 & Walla Walla \\
\hline $7-7$ & 29 & $<1$ & Burbarik \\
\hline $7-11$ & 21. & 44 & Fasco \\
\hline $7-12$ & 33 & $<1$ & Moses Lake \\
\hline $7-14$ & 33 & 31 & Walla Walla \\
\hline $7-18$ & 37 & 7 & Hermiston, Creson \\
\hline $7-20$ & 17 & $<1$ & Erosser \\
\hline $7-21$ & 19 & $<1$ & Elcopia \\
\hline $7-25$ & 26 & 5 & Wallia Walla \\
\hline $7-25$ & 25 & 3 & Hermiscor, Oreson \\
\hline $7-26$ & 20 & $<1$ & Warder \\
\hline $8-1$ & 26 & 2 & Prosser \\
\hline
\end{tabular}


APPENDIX G

TABLE I (Contirued)

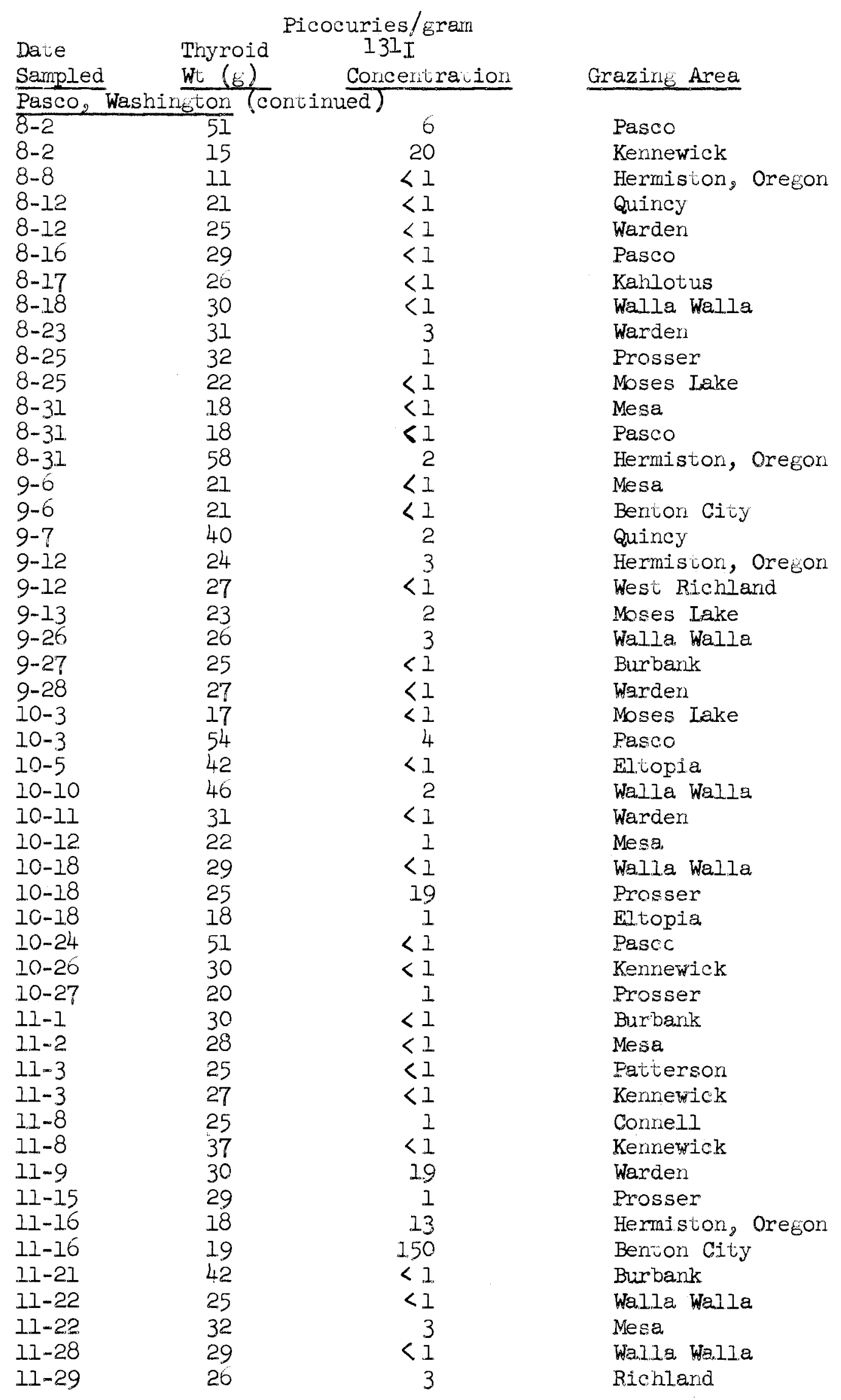


APPENDIX G

\section{TABLE I (Concinued)}

\begin{tabular}{|c|c|c|}
\hline \\
\hline \multicolumn{2}{|r|}{ Tryroid } & \\
\hline Sarmpled & W $(g)$ & Concentration \\
\hline \multicolumn{3}{|c|}{ 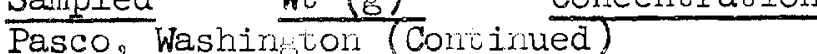 } \\
\hline $12-1$ & 26 & $<1$ \\
\hline $12-6$ & 31 & $<\bar{I}$ \\
\hline $12-6$ & 41 & 7 \\
\hline $.12-6$ & 28 & 6 \\
\hline $12-12$ & 33 & $<1$ \\
\hline $12-12$ & 42 & $<i$ \\
\hline $12-13$ & 41 & 10 \\
\hline $12-20$ & 26 & $<1$ \\
\hline $12-20$ & 35 & $<1$ \\
\hline $12-21$ & 26 & $<1$ \\
\hline $12-27$ & 48 & $<1$ \\
\hline $12-29$ & 37 & $<1$ \\
\hline $12-29$ & 24 & $<1$ \\
\hline
\end{tabular}

Grazing Area

Kernewick

Kahilotus

Walla Walla

Pasco

Walla Walla

Kenneyick

Prosser

Mesa.

Prosser

Connell.

Walla Walla

Kennetrick

Milion-Freewaler, Ore.

Moses Lake, Washington

\begin{tabular}{|c|c|c|c|}
\hline $1-15$ & 32 & $<1$ & Marlir \\
\hline $1-1.5$ & 23 & 2 & Warden \\
\hline $1-15$ & 52 & $<1$ & Ephrata \\
\hline $.1-15$ & 33 & $<1$ & Moses Lake \\
\hline $1-15$ & 27 & 3 & Moses Iake \\
\hline $1-29$ & $5 ?$ & $<1$ & Warden \\
\hline $1 .-29$ & 46 & $<1$ & Warden \\
\hline $1-29$ & 20 & 18 & Warden \\
\hline $1-29$ & 48 & $<1$ & Moses Lake \\
\hline $1-29$ & 63 & $<1$ & Moses Lake \\
\hline $2-12$ & 32 & $<I$ & Hooper \\
\hline $1-12$ & 59 & $<1$ & Moses Lake \\
\hline $2-12$ & 34 & $<1$. & Warden \\
\hline $2-12$ & 35 & $<1$ & Warden \\
\hline $2-12$ & 38 & 1 & Warden \\
\hline $2-24$ & 52 & $<1$ & Moses Iake \\
\hline $2-24$ & 44 & $<1$ & Mbsee Iake \\
\hline $2-24$ & 39 & $<1$ & Conneli \\
\hline $2-24$ & 34 & $<1$ & Stratford \\
\hline $2-26$ & 38 & $<1$ & Royal city \\
\hline $3-.12$ & 31 & 1 & Ritzville \\
\hline $3-12$ & 20 & 4 & Ritzvilale \\
\hline $3-12$ & 41 & 1 & Odessa \\
\hline $3-15$ & 25 & $<1$ & Moses Lake \\
\hline $3-15$ & 49 & $<1$ & Mbses Ink: \\
\hline $4-2$ & 33 & $<1$ & Grand Coviee \\
\hline $4-2$ & 42 & $<1$ & Ephrata \\
\hline $4-2$ & 33 & $<.1$ & Moses Iake \\
\hline $4-2$ & 28 & $<1$ & Moses Lake \\
\hline $4-2$ & 21 & $<1$ & othello \\
\hline $4 .-14$ & 41 & 6 & Otheilo \\
\hline $4-14$ & 35 & $<1$ & othe310 \\
\hline $4-14$ & 27 & $<1$ & Quincy \\
\hline $4-.14$ & 30 & 4 & Iind \\
\hline
\end{tabular}


APEEIDIX $G$

TABIE 1 (Continued)

\begin{tabular}{|c|c|c|c|c|}
\hline \multicolumn{4}{|c|}{ Picocuries/gram } & \\
\hline Sampled & Wt $(8)$ & Concertration & Grazing Area. & \\
\hline Moses I & Washingto & ontinued) & & \\
\hline$\sqrt{4-14}$ & 45 & 2 & Iind & \\
\hline $4-28$ & 25 & $<I$ & Moses Iake & \\
\hline $4-28$ & 33 & $<1$ & Moses Luke & \\
\hline $4-28$ & 21 & $<1$ & Quiney & \\
\hline $4-28$ & 18 & $<1$ & Quiney & \\
\hline $4-28$ & 35 & 1 & Odessa & \\
\hline $5 \approx 14$ & 28 & 4 & Otheilo & \\
\hline $5-14$ & 34 & 2 & Quincy & \\
\hline $5-14$ & 35 & 3 & Mansfield & \\
\hline $5-.2+$ & 32 & 2 & Hartline & \\
\hline $5-14$ & 27 & 3 & Moses Lake & \\
\hline $5 \infty 27$ & 61 & 5 & Kahlotis & \\
\hline $5-27$ & 25 & $<1$ & Kainlotus & \\
\hline $5-27$ & 48 & $<1$ & odessa & \\
\hline $5-27$ & 41 & 19 & Moses Lake & \\
\hline $5-2 ?$ & 39 & $<i$ & $\mathrm{Msses}$ Luke & \\
\hline 6.16 & 26 & 40 & othe1.10 & \\
\hline $6 \cdot 16$ & 47 & 4 & Othelis & \\
\hline $6-16$ & 54 & 1 & Mses Iake & \\
\hline $6-16$ & 60 & 36 & Wheelex & \\
\hline $6 \cdots 16$ & 38 & $7^{\prime}$ & Wheeder & \\
\hline 6.25 & 42 & 0 & otheilo & \\
\hline 6.25 & 23 & $1]$ & otheido & \\
\hline 6.25 & 27 & 24 & othe 910 & \\
\hline $6-25$ & 32 & $<1$ & Eltopia & \\
\hline $6-25$ & 29 & 2 & Moses Iake & \\
\hline $7-6$ & 52 & 3 & Moses Irike & \\
\hline $7 \times 6$ & 41 & 7 & Moses Jake & \\
\hline $7 \times 6$ & 45 & 1 & Moss Iake & \\
\hline $7=6$ & 23 & 3 & othelio & \\
\hline $7 \times 6$ & 43 & 42 & 0 thein 10 & \\
\hline $7-23$ & 45 & 2 & Ritzvizle & \\
\hline $7-23$ & 38 & 21 & Ritzvis:e & \\
\hline $7 \infty 23$ & 34 & 3 & Moses Lake & \\
\hline $7-23$ & 34 & 25 & Moes Taks & \\
\hline $7=23$ & 49 & 19 & Mosed Dake & \\
\hline $8-\infty 6$ & 35 & 4 & Moses Lake & \\
\hline $8 \cdot 6$ & 40 & 2 & Moses Isake & \\
\hline $8-6$ & 32 & 2 & Moses Lake & \\
\hline $8-6$ & 31 & $<1$ & Moses Iake & \\
\hline $8=6$ & 37 & 2 & Ia Crosse & \\
\hline $8 \ldots 19$ & 41 & 3 & Ioomis & \\
\hline $8-19$ & 58 & 4 & Ioomi:- & \\
\hline $8-1.9$ & $4+4$ & 1 & Lomis & \\
\hline $8 \times 19$ & 41 & 4 & Loomis & \\
\hline $8 \times 19$ & 72 & $<2$ & Lacrosse & \\
\hline $9-1$ & 50 & $<1$ & Hermiston, & Oregon \\
\hline $9 \times 1$ & 38 & $<1$ & Moses Iake & \\
\hline $9=1$ & 38 & 1 & Moses Lake & \\
\hline
\end{tabular}


APPENDIX G

TABLE I (Continued)

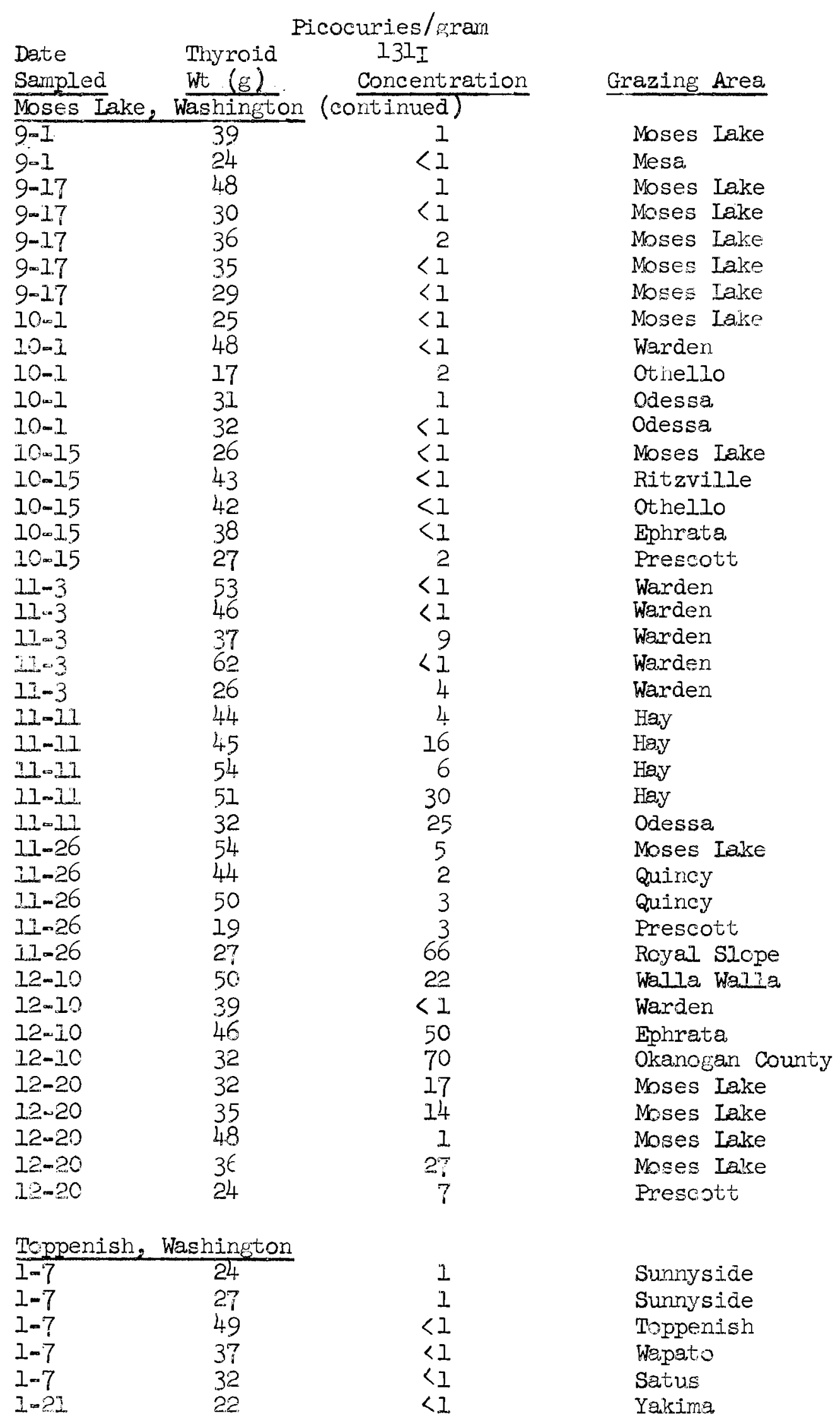


APEENDTE G

TABIE I (Continued)

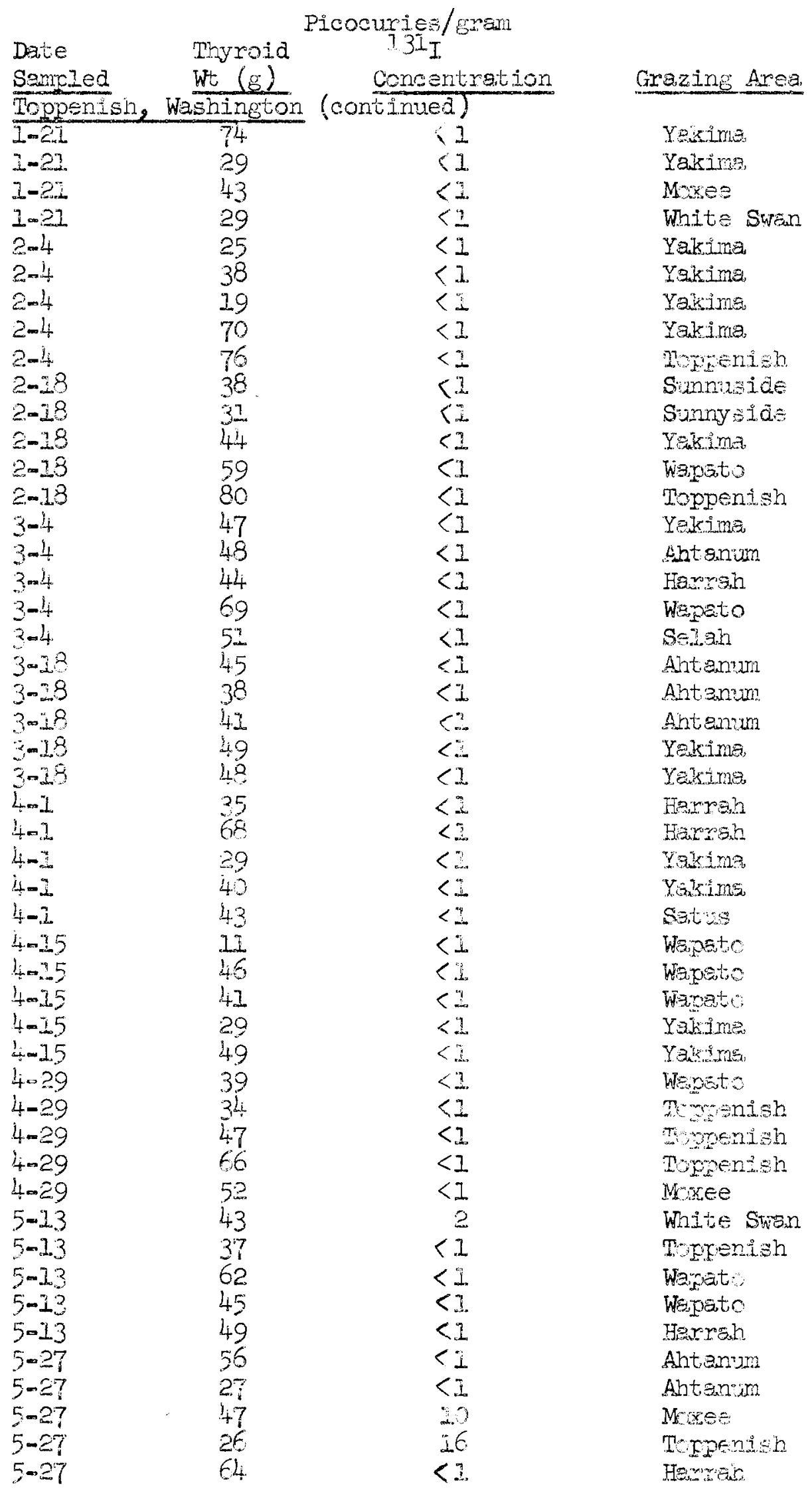


APPENDIX G

TABLE 1 ( Continued)

Picocuries/gram

\begin{tabular}{|c|c|c|c|}
\hline $\begin{array}{l}\text { Date } \\
\text { Sampled }\end{array}$ & $\begin{array}{l}\text { Thyroid } \\
\text { Wt }(g) \\
\text { Washington }\end{array}$ & $\begin{array}{c}13 I_{I} \\
\text { Concentration }\end{array}$ & Grazing Area \\
\hline Toppenish, & Washington & (continued) & \\
\hline $6-10$ & 40 & 2 & Yakima \\
\hline $6-10$ & 23 & 1 & Yakima \\
\hline $6-10$ & 29 & $<1$ & Toppenish \\
\hline $6-10$ & 49 & 12 & Toppenish \\
\hline $6-10$ & 43 & 16 & Moxee \\
\hline $6-24$ & 38 & $<1$ & White Swan \\
\hline $6-24$ & 45 & 3 & Ahtanum \\
\hline $6-34$ & 34 & 2 & Aht $=$ nimn \\
\hline $6-24$ & 32 & $<1$ & Moxee \\
\hline $6-24$ & 45 & 13 & Moxee \\
\hline $7-8$ & 51 & 5 & Yakima \\
\hline $7-8$ & 37 & 1 & Yakima \\
\hline $7-8$ & 82 & 6 & Yakima. \\
\hline $7-8$ & 42 & $<1$ & Yakima \\
\hline $7-8$ & 98 & 7 & Yakima \\
\hline $7-22$ & 55 & 6 & Toppenish \\
\hline $7-22$ & 42 & 2 & Ahtanum \\
\hline $7-22$ & 65 & 4 & Ahtanum \\
\hline $7-22$ & 29 & 1 & White Swan \\
\hline $8-12$ & 47 & $<1$ & Yakima \\
\hline $8-12$ & 59 & 1 & Toppenish \\
\hline $8-12$ & 47 & $<1$ & Toppenish \\
\hline $8-12$ & 57 & 2 & Wapato \\
\hline $8-12$ & 48 & $<1$ & Moxee \\
\hline $9-9$ & 36 & $<1$ & White Swan \\
\hline $9-9$ & 53 & $<1$ & Toppenish \\
\hline $9-9$ & 24 & $<1$ & Brownetown \\
\hline $9-9$ & 39 & $<1$ & Wiley City \\
\hline $9-9$ & 75 & $<1$ & Yakima \\
\hline $9-23$ & 54 & 7 & Toppenish \\
\hline $9-23$ & 44 & $<1$ & Fakima \\
\hline $9-23$ & 35 & $<1$ & Brownstown \\
\hline $9-23$ & 43 & $<1$ & Bickleton \\
\hline $9-23$ & 53 & $<1$ & Bickleton \\
\hline $10-7$ & 42 & $<1$ & Toppenish \\
\hline $10-7$ & 56 & $<1$ & Wapato \\
\hline $10-7$ & 37 & $<1$ & Moxee \\
\hline $10-21$ & 35 & $<1$ & Yakima \\
\hline $10-21$ & 39 & $<1$ & White Swan \\
\hline $10-21$ & 71 & $<1$ & Wapato \\
\hline $10-21$ & 52 & $<1$ & Granger \\
\hline $10-21$ & 50 & $<1$ & Zillah \\
\hline $11-4$ & 44 & $<1$ & Satus \\
\hline $11-4$ & 33 & $<1$ & White Swan \\
\hline $11-4$ & 37 & $<1$ & Wapato \\
\hline $11-4$ & 53 & $<1$ & Moxee \\
\hline $11-4$ & 43 & $<1$ & Ahtanum \\
\hline $11-18$ & 48 & 62 & Zillah \\
\hline $11-18$ & 69 & 10 & Wapato \\
\hline $11-18$ & 38 & 18 & Toppenish \\
\hline
\end{tabular}


$\triangle P F E T D I X 9$

TABIE I (Continued)

\begin{tabular}{|c|c|c|}
\hline \multicolumn{3}{|c|}{ Ficoouries/gran } \\
\hline $\operatorname{san} x d e c$ & Wt $(E)$ & Conuentration \\
\hline Toppenz= & Washingto & ntinued) \\
\hline $21-28$ & $5 \mathrm{I}$ & $<I$ \\
\hline $21-18$ & 4 & 98 \\
\hline $12-2$ & 39 & $<I$ \\
\hline $12=2$ & 46 & 2 \\
\hline $1 \geq-2$ & 78 & $<3$ \\
\hline$I=-?$ & 56 & $<i$ \\
\hline $12-2$ & 208 & $<1$ \\
\hline $12-16$ & 52 & $<3$. \\
\hline $12-16$ & 55 & $<$. \\
\hline $12-.16$ & 45 & $<1$ \\
\hline $1 \geq-i 6$ & 68 & $<$ \\
\hline $22-16$ & 48 & $<1$ \\
\hline 12.23 & 32 & $<1$ \\
\hline $12-23$ & 38 & $<$ \\
\hline $12-23$ & 59 & 2 \\
\hline$I=-23$ & 53 & $<1$ \\
\hline $12-23$ & 43 & $?$ \\
\hline
\end{tabular}

Waije Weile, Washington

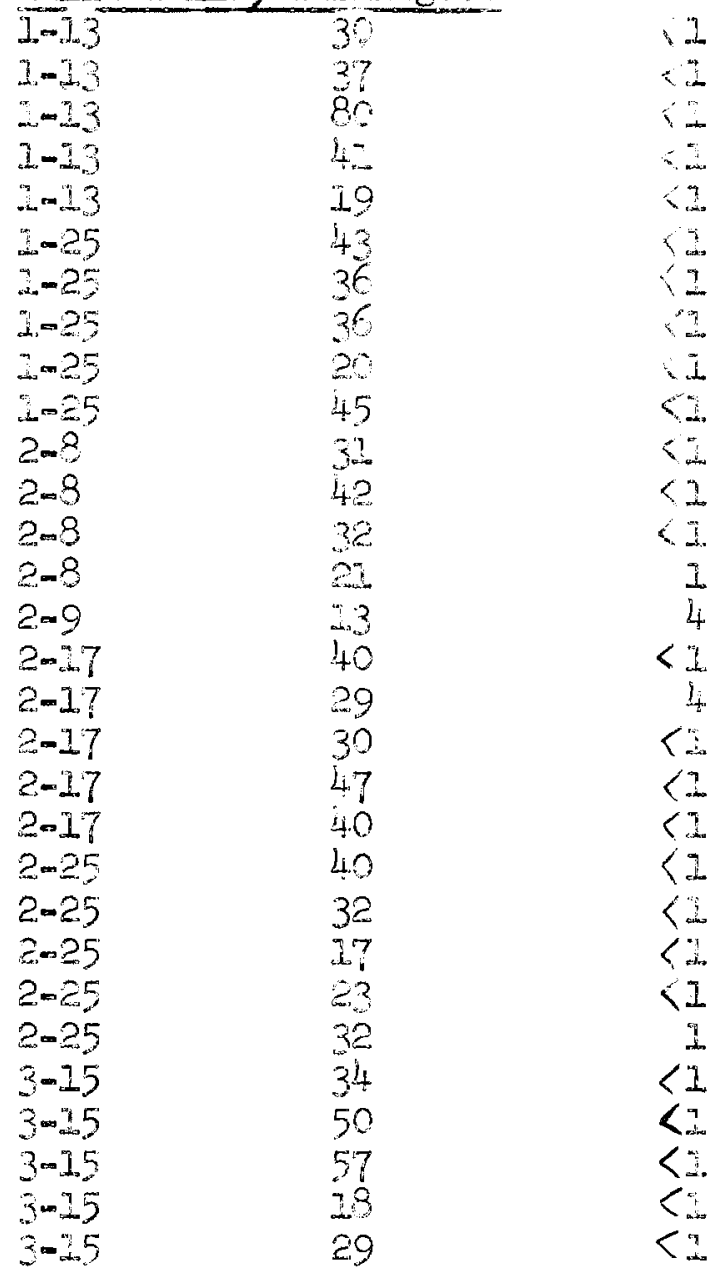

Grazing Area

Harrein

Naches

Wapato

Wejpito

Yaikina

Yakinn

Yakifima

Yeikime

Ahtsn:m

Artanam

Topnenish

Somb

Topyenish

toppenish

Torpanish

Ahtengon

Abten:m

W29 5 W 130

Weg $\mathrm{WE}$ -

WoIIa WEIIA

Weila Wata

WiLE Wina.

Presoot

Freseott

Trnapine, Cregon

Lewan

Iowden

Iswan

Fosec

Psoce

Milton Freewatex, Ops.

Lowden

WeIIa WaIs

Waine W5210

Waja Wajia

Waİa WE:

Stsrobusix

Touchet

Trichet

WIİ Wala

Wanis Was.;

Waíg WEILA

Toushet

Toushet

Inimgen

wordsn

Calege Flece 
APFEIDIX G

TABLE I (Continued)

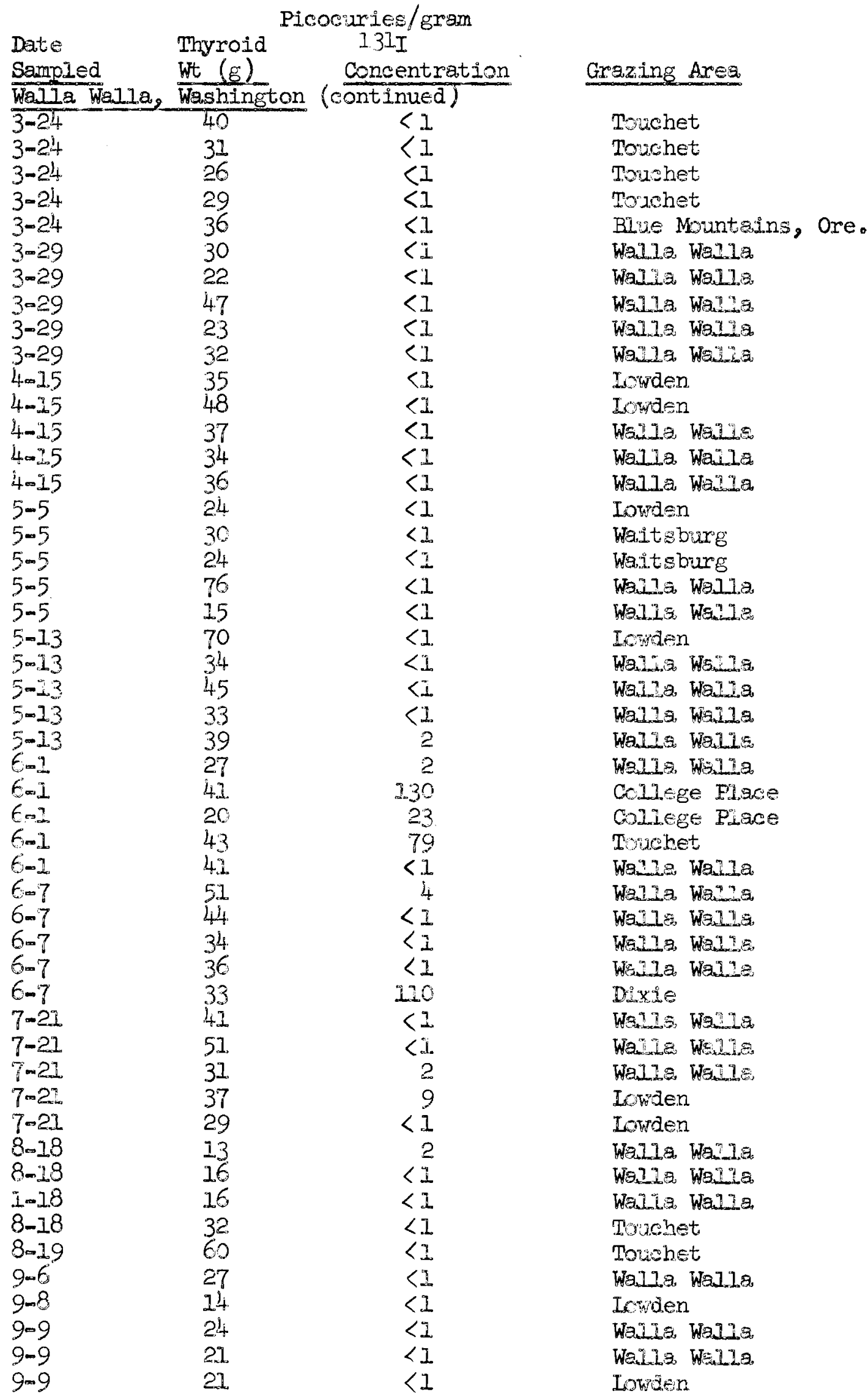




\begin{tabular}{|c|c|c|c|}
\hline \multicolumn{4}{|c|}{$\begin{array}{c}\text { APPENDIX } G \\
\text { TABIE I (Cont nused) }\end{array}$} \\
\hline \multicolumn{4}{|c|}{ Picouvries/gram } \\
\hline Sampled & Wt $(g)$ & wneentration & Grazing Area \\
\hline \multicolumn{4}{|c|}{ WEjIa Wajla, WEshington (ontinued) } \\
\hline $9-21$ & 28 & $<2$ & Milton-Freewater, Ore. \\
\hline $9-21$ & 28 & $<1$ & Milton-Freewater, Ore. \\
\hline $9-21$ & 46 & $<i$ & Towoset \\
\hline $9-21$ & $2 i$ & $<1$ & Iniden \\
\hline $9-21$. & 2.7 & $<2$ & Iowien \\
\hline $10=14$ & 24 & $<2$ & Prosest \\
\hline $10 \cos 4$ & 33 & $<1$ & Whom \\
\hline $10-14$ & 25 & $<$ & Lowden \\
\hline $20=-4$ & 29 & 2 & Milto-Ereewater, ore. \\
\hline $10-14$ & 42 & $<1$ & Tozobet \\
\hline $12-10$ & 29 & 5 & 0,11080 Rsgee \\
\hline $17-.20$ & 32 & 2 & Co $20 \mathrm{ge}$ PIgne \\
\hline $11-10$ & 34 & 3 & $L, w d \in n$ \\
\hline $12=10$ & 23 & $i$ & WaIIa Waina \\
\hline $1=-20$ & 50 & 25 & W2:1E 6529 \\
\hline $11-22$ & 71 & $<1$ & Touchet \\
\hline $12-22$ & 52 & $<\bar{l}$ & Lowaen \\
\hline $72-22$ & 58 & 88 & Bive Murtains, Ore. \\
\hline $2-22$ & 58 & 94 & Blue Muntains, Ore. \\
\hline $2-22$ & 20 & 150 & Waile We's: \\
\hline $23-2$ & 89 & $<1$ & WE" 25 Weine \\
\hline 202 & 16 & 35 & WeIIa Wasia \\
\hline $12=3$ & 32 & 6 & Wras WaI \\
\hline $22-3$ & 34 & $<2$ & WIIZ WaIIa \\
\hline $22-2$ & 36 & 6 & Wh 10 WaIs \\
\hline $22-15$ & 53 & $<1$ & Msito-Freenater, ore. \\
\hline $12-5$ & 33 & 6 & WE \\
\hline $2=-25$ & 39 & $<2$ & WE- \\
\hline $20-5$ & 17 & $<3$ & Wa $=$ Wo \\
\hline $12-15$ & 26 & 5 & Wa:Sa Wa \\
\hline \multicolumn{4}{|c|}{ Wenatchee, Washington } \\
\hline $1=\$ 9$ & 71 & $<1$ & Dougas County \\
\hline$I=19$ & 49 & $<2$ & Douglas County \\
\hline $1-19$ & 52 & $<3$ & Dougla:s Crunty \\
\hline $1=9$ & 46 & $<1$ & Dougasas chunty \\
\hline$\pm=19$ & 48 & $<2$ & Douglas County \\
\hline $2-2$ & 38 & $<1$ & Dturgas county \\
\hline $2-2$ & 43 & $<2$ & Drogsas county \\
\hline $2-2$ & 26 & $<1$ & Dougies county \\
\hline $2-2$ & 34 & $<1$ & Duglas connty \\
\hline $2-2$ & 49 & $<\Sigma$ & DUgias oranty \\
\hline $2-20$ & 40 & $<2$ & Che 20 county \\
\hline $2-22$ & 54 & $<3$ & WaIIs WaIs Couty \\
\hline $2-22$ & 51 & $<1$ & Wa 1 s wos a cunty \\
\hline $2-22$ & 98 & $<1$ & Spokane \\
\hline $2-22$ & 34 & $<2$ & Grant cyunty \\
\hline $3-7$ & 37 & $<2$ & Grant county \\
\hline $3-7$ & 4.1 & 2 & Grant cooty \\
\hline
\end{tabular}


APFEIVIX G

TABLE 1 (Continued)

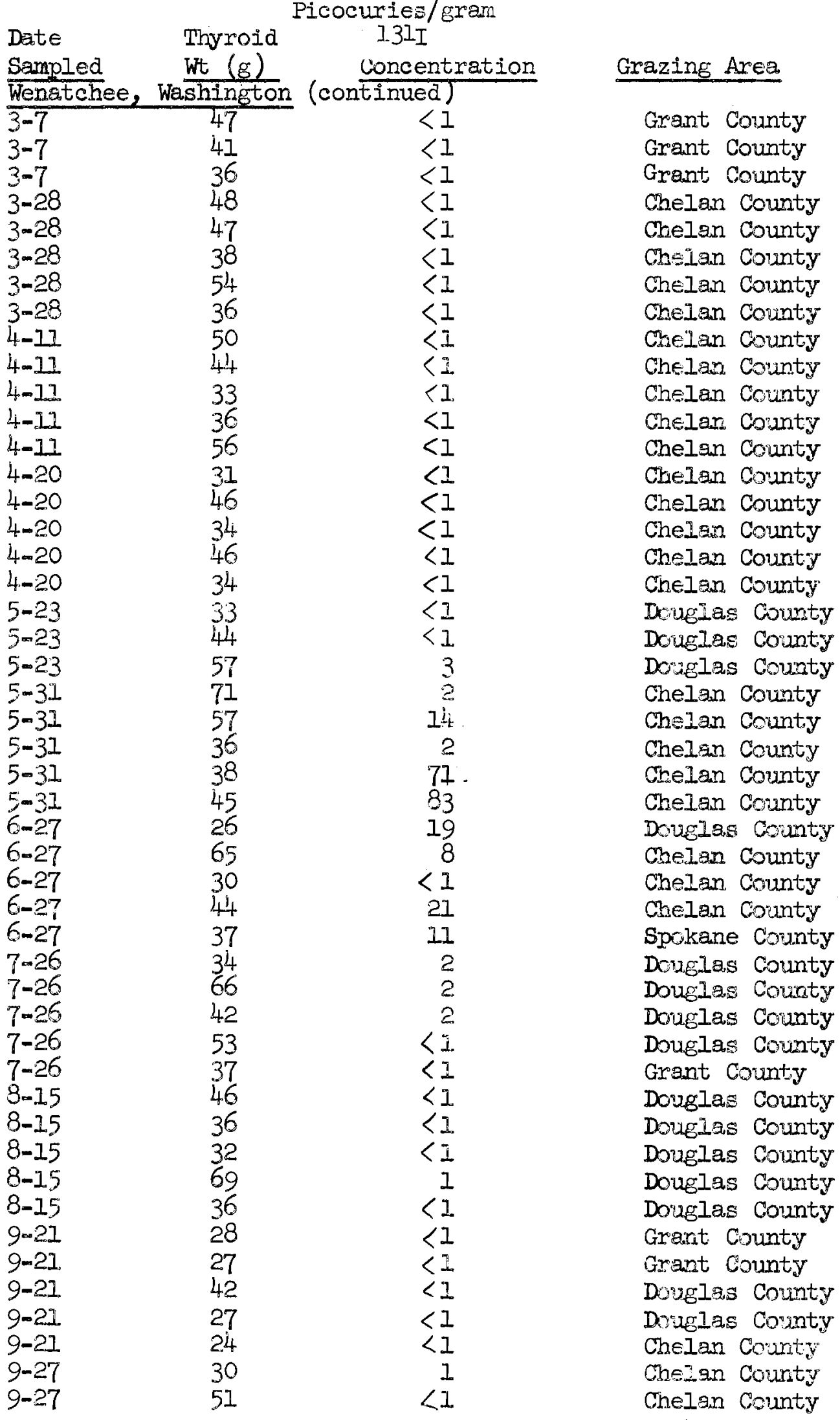




\section{APPENDIX G \\ TABLE I (Continued)}

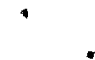

\begin{tabular}{|c|c|c|c|}
\hline \multicolumn{4}{|c|}{ Picocuries/gram } \\
\hline Date & Thyroid & $132 I$ & \\
\hline Sampled & Wt (g) & Coneentration & Grazing Area \\
\hline Wenatchee & Washington & atinued) & \\
\hline$\overline{9-27}$ & 26 & $i$ & Chelan County \\
\hline $9-27$ & 30 & $<\overline{1}$ & Chelan County \\
\hline $9-27$ & 40 & $<1$ & Chelan County \\
\hline $10-10$ & 31 & $<1$ & Chelan County \\
\hline $10-10$ & 40 & $<1$ & Melan County \\
\hline $10-10$ & 30 & $<1$ & Chelan County \\
\hline $10-10$ & 35 & $<1$ & Chelan County \\
\hline $16-10$ & 24 & $<1$ & Douglas County \\
\hline
\end{tabular}


BNWL-439 APP

\author{
APPENDIX H \\ MEASUREMENTS OF EXTERNAL RADIATION EXPOSURE
}




\section{APPENDIX H}

TABLE 1

IONIZATION CHAMBER MEASUREMENTS FOR THE HANEORD RESERVATION AND RICHLAND - 1966

\begin{tabular}{|c|c|c|}
\hline \multirow{2}{*}{$\begin{array}{l}\text { Measurement } \\
\text { Period }\end{array}$} & \multicolumn{2}{|c|}{ Exposure Rate mR/Day } \\
\hline & Hanford & Richland \\
\hline $12 / 29 / 65-1 / 3 / 66$ & Lost & 0.38 \\
\hline $1 / 3-1 / 5$ & 0.41 & 0.33 \\
\hline $1 / 5=1 / 7$ & 0.34 & 0.29 \\
\hline $1 / 7-1 / 10$ & 0.46 & 0.26 \\
\hline $1 / 10=1 / 12$ & 0.32 & 0.28 \\
\hline $1 / 12-1 / 17$ & 0.42 & 0.32 \\
\hline $1 / 17-1 / 19$ & 0.45 & 0.31 \\
\hline $1 / 19-1 / 2 i$ & 0.36 & 0.33 \\
\hline $1 / 21-1 / 24$ & 0.53 & 0.38 \\
\hline $1 / 24=1 / 26$ & 0.40 & 0.24 \\
\hline $1 / 26-1 / 28$ & 0.43 & 0.31 \\
\hline $1 / 28-1 / 31$ & 0.52 & 0.31 \\
\hline $1 / 31-2 / 2$ & 0.34 & 0.28 \\
\hline $2 / 2-2 / 4$ & 0.44 & 0.25 \\
\hline $2 / 4-2 / 7$ & 0.55 & 0.38 \\
\hline $2 / 7-2 / 9$ & Lost & Lost \\
\hline $2 / 9-2 / 10$ & 0.25 & 0.21 \\
\hline $2 / 10=2 / 14$ & 0.48 & 0.31 \\
\hline $2 / 14-2 / 16$ & 0.43 & 0.29 \\
\hline $2 / 16-2 / 18$ & 0.33 & 0.19 \\
\hline $2 / 18-2 ! 21$ & 0.47 & 0.31 \\
\hline $2 / 21-2 / 23$ & 0.41 & 0.27 \\
\hline $2 / 23-2 / 25$ & 0.44 & 0.33 \\
\hline $2 / 25-2 / 28$ & 0.37 & 0.28 \\
\hline $2 / 28-3 / 2$ & 0.36 & 0.29 \\
\hline $3 / 2-3 / 4$ & 0.39 & 0.26 \\
\hline $3 / 4=3 / 7$ & 0.45 & 0.32 \\
\hline $3 / 7-3 / 9$ & 0.33 & 0.20 \\
\hline $3 / 9-3 / 11$ & 0.32 & 0.26 \\
\hline $3 / 11-3 / 14$ & 0.39 & 0.30 \\
\hline $3 / 14-3 / 16$ & 0.42 & 0.35 \\
\hline $3 / 16-3 / 18$ & 0.37 & 0.28 \\
\hline $3 / 18-3 / 21$ & 0.31 & 0.30 \\
\hline $3 / 21-3 / 23$ & 0.30 & 0.19 \\
\hline $3 / 23-3 / 25$ & 0.33 & 0.27 \\
\hline $3 / 25-3 / 28$ & 0.37 & 0.31 \\
\hline $3 / 28-3 / 30$ & 0.37 & 0.27 \\
\hline
\end{tabular}
Measurement Period

$3 / 30-4 / 1$

$4 / 1-4 / 4$

$4 / 4-4 / 6$

$4 / 6-4 / 8$

$4 / 8-4 / 11$

$4 / 11-4 / 15$

$4 / 15-4 / 18$

$4 / 18-4 / 20$

$4 / 20-4 / 22$

$4 / 22-4 / 25$

$4 / 25-4 / 27$

$4 / 27-4 / 29$

$4 / 29-5 / 2$

$5 / 2-5 / 4$

$5 / 4-5 / 6$

$5 / 6-5 / 9$

$5 / 9-5 / 11$

$5 / 11 \cdot 5 / 13$

$5 / 13-5 / 16$

$5 / 16-5 / 18$

$5 / 18-5 / 20$

$5 / 20=5 / 23$

$5 / 23=5 / 25$

$5 / 2.5-5 / 27$

$5 / 27-5 / 31$

$5 / 31-6 / 3$

$6 / 3-6 / 6$

$6 / 6-6 / 8$

$6 / 8-6 / 10$

$6 / 10-6 / 13$

$6 / 13-6 / 15$

$6 / 15=6 / 17$

$6 / 17-6 / 20$

$6 / 20-6 / 22$

$6 / 22-6 / 24$

$6 / 24=6 / 27$

$6 / 27=6 / 29$
Exposure Rate mR/Day

Hanford Richland

0.27

0.23

0.36

0.26

0.34

0.22

0.35

0.29

0.31

0.19

0.35

0.28

0.30

0.18

0.34

0.27

0.29

0.21

0.31

0.27

0.30

0.26

0.28

0.23

0.20

0.32

0.22

0.19

0.26

0.17

0.28

Lost

0.20

Lost

0.38

0.29

0.37

0.32

0.38

0.31

0.32

0.22

0.31

0.23

0.33

0.33

0.43

0.33

0.30

0.33

0.44

0.30

0.32

0.32

0.33

Lost

0.34

0.31
0.21

0.23

0.29

0.37

0.32

0.22

0.28

0.24

0.28

0.20

0.23

0.27

Lost

0.31

0.29

0.24 


\author{
APPENDIX $\mathrm{H}$ \\ Table 1 (continued)
}

Measurement

Period

$$
6 / 29-7 / 1
$$

$7 / 1-7 / 5$

$7 / 5-7 / 15$

$7 / 15-7 / 18$

$7 / 18-7 / 20$

$7 / 20-7 / 22$

$7 / 22-7 / 25$

$7 / 25-7 / 27$

$7 / 27-7 / 29$

$7 / 29-8 / 1$

$8 / 1-8 / 3$

$8 / 3-8 / 5$

$8 / 5-8 / 10$

$8 / 10-8 / 12$

$8 / 12-8 / 15$

$8 / 15-8 / 17$

$8 / 17-8 / 19$

$8 / 19-8 / 22$

$8 / 22-8 / 24$

$8 / 24-8 / 26$

$8 / 26-8 / 29$

$8 / 29-9 / 1$

$9 / 1-9 / 2$

$9 / 2-9 / 6$

$9 / 6-9 / 9$

$9 / 9-9 / 12$

$9 / 12-9 / 14$

$9 / 14-9 / 16$

$9 / 16-9 / 19$

$9 / 19-9 / 21$

$9 / 21-9 / 26$

$9 / 26-9 / 28$

$9 / 28-9 / 30$

$9 / 30-10 / 3$
Exposure Rate mR/Day

Hanford Richland

$$
0.32
$$

Lost

Lost

0.32

0.28

0.26

0.39

0.23

0.25

0.21

0.29

0.15

0.34

Lost

0.31

0.25

0.21

0.34

0.29

0.30

0.37

0.32

0.16

0.35

0.28

0.36

0.34

Lost

0.36

0.29

0.36

0.31

0.30

0.39
0.23

Lost

0.16

0.18

0.21

0.29

0.21

0.20

0.18

0.18

0.22

0.16

0.27

Lost

0.20

0.39

Lost

0.31

0.23

0.27

0.25

0.30

0.15

0.29

0.24

0.42

0.24

Lost

0.23

0.41

0.37

0.28

0.21

0.42
Me as urement Period

$10 / 3-10 / 5$

$10 / 5-10 / 7$

$10 / 7-10 / 11$

$10 / 11-10 / 13$

$10 / 13-10 / 17$

$10 / 17-10 / 19$

$10 / 19-10 / 21$

$10 / 21-10 / 24$

$10 / 24-10 / 28$

$10 / 28-10 / 31$

$10 / 31-11 / 2$

$11 / 2-11 / 4$

$11 / 4-11 / 7$

$11 / 7-11 / 9$

$11 / 9-11 / 11$

$11 / 11-11 / 14$

$11 / 14-11 / 16$

$11 / 16-11 / 18$

$11 / 18-11 / 21$

$11 / 21-11 / 23$

$11 / 23-11 / 28$

$11 / 28-11 / 30$

$11 / 30-12 / 2$

$12 / 2-12 / 5$

$12 / 5-12 / 7$

$12 / 7-12 / 9$

$12 / 9-12 / 12$

$12 / 12-12 / 14$

$12 / 14-12 / 16$

$12 / 16-12 / 19$

$12 / 19-12 / 21$

$12 / 21-12 / 27$

$12 / 27-12 / 30$
Exposure Rate mR/Day

Hanford Richland

0.30

0.23

0.39

0.41

0.57

0.30

0.40

0.34

0.35

0.41

0.49

0.42

0.49

0.42

0.30

0.36

0.40

0.37

0.33

0.45

0.33

0.37

0.39

0.48

0.46

0.35

0.37

0.39

0.36

0.40

0.44

0.35

Lost

0.40
0.37

0.31

0.50

0.20

0.32

0.20

0.33

0.28

0.43

0.26

0.39

0.28

0.41

0.30

0.33

0.27

0.32

0.31

0.24

0.28

0.34

0.33

0.33

0.23

0.33

0.32

0.26

0.24

0.33

0.26

0.33

0.28 
APPENDTX H

Table 2

IONIZATION CHAMBER MEASUREMENTS IN THE COLUMBIA RIVER - 1966

Measurement Exposure Rate Period $\mathrm{mR} /$ Day

Measurement

Period
Exposure Rate $\mathrm{mR}$ / Day
Measurement Period
Exposure Rate $\mathrm{mR} /$ Day

Vernita Bridge

$1 / 4-1 / 11 \quad 0.31$

$1 / 11=1 / 18 \quad 0.44$

$1 / 18-1 / 25 \quad 0.83$

$1 / 25=2 / 1 \quad 1.00$

$2 / 1-2 / 22 \quad$ Lost

$2 / 22-3 / 1 \quad 0.96$

$3 / 1-3 / 8 \quad 0.28$

$3 / 8-3 / 15 \quad 0.72$

$3 / 15-3 / 22$

$3 / 22-3 / 29$
0.32

0.48

old Hanford Ferry

$12 / 28 / 65-1 / 4 / 66 \quad 4.95$

$1 / 4-1 / 11 \quad 3.95$

$1 / 11=1 / 18 \quad 7.37$

$1 / 18-1 / 25 \quad 4.67$

$1 / 25=2 / 1 \quad 5.84$

$2 / 1-2 / 10 \quad 6.45$

$2 / 10-2 / 17 \quad 4.93$

$2 / 17=2 / 22 \quad 2.11$

$2 / 22-3 / 1 \quad 4.50$

$3 / 1=3 / 8 \quad 5.28$

$3 / 8-3 / 15 \quad 4.86$

$3 / 17=3 / 22 \quad 4.56$

$3 / 22=3 / 29 \quad 5.81$

$3 / 29-4 / 5 \quad 6.19$

$4 / 5=4 / 12 \quad 7.30$

Ringold

$\begin{array}{ll}12 / 28 / 65-1 / 4 / 66 & 3.97 \\ 1 / 4-1 / 11 & 2.88 \\ 1 / 11-1 / 18 & 2.25 \\ 1 / 18=1 / 25 & 3.36 \\ 1 / 25-2 / 1 & \text { Lost } \\ 2 / 1=2 / 8 & 3.89 \\ 2 / 8=2 / 15 & 4.05 \\ 2 / 15=2 / 22 & 2.74 \\ 2 / 22=3 / 1 & 4.30\end{array}$

$$
\begin{aligned}
& 3 / 29-4 / 5 \\
& 4 / 5-4 / 12 \\
& 4 / 12=4 / 19 \\
& 4 / 19=4 / 26 \\
& 4 / 26=5 / 5 \\
& 5 / 5=5 / 17 \\
& 5 / 17=5 / 24 \\
& 5 / 24=5 / 31 \\
& 5 / 31=6 / 7 \\
& 6 / 7=6 / 14
\end{aligned}
$$

0.43

0.53

0.47

0.91

0.16

Lost

0.10

0.86

0.29

0.41
$6 / 14-6 / 21$

$6 / 21-6 / 28$

$6 / 28-7 / 5$

$7 / 5-7 / 19$

$7 / 19-8 / 29$

$8 / 29-9 / 6$

$9 / 6-9 / 12$

$9 / 19=9 / 27$

$9 / 27 \cdot 10 / 4$

$10 / 18-10 / 25$
1.28

0.35

0.09

0.60

Lost

0.61

0.87

1. 72

0.31

$2 \cdot 34$
$4 / 12=4 / 19$

$4 / 19-5 / 3$

$5 / 3-5 / 5$

$5 / 5=5 / 17$

$5 / 17-5 / 24$

$5 / 24-5 / 31$

$5 / 31-6 / 7$

$6 / 7-6 / 14$

$6 / 14-6 / 21$

$6 / 21-6 / 28$

$6 / 28=7 / 5$

$7 / 5=7 / 15$

$7 / 15-7 / 19$

$8 / 22-8 / 29$

$8 / 29-9 / 6$

6.06
Off scale
5.73
Lost
3.83
2.19
2.68
1.51
2.00
3.42
5.62
1.68
0.41
4.39
5.22

$9 / 6-9 / 12$

$9 / 12-9 / 19$

$9 / 19-9 / 27$

$9 / 27-10 / 4$

$10 / 4-10 / 11$

$10 / 11-10 / 18$

$10 / 18-10 / 25$

$10 / 25-11 / 8$

$11 / 8-11 / 15$

$11 / 15-11 / 22$

$11 / 22-11 / 29$

$11 / 29 \times 12 / 6$

$12 / 6-12 / 13$

$12 / 13-12 / 20$

$12 / 20=12 / 28$
2.70

4.20

4.33

2.62

2.31

1.94

2. 06

2. 52

6.53

2.91

5.50

1.49

2.38

2.53

3.40
$3 / 1-3 / 8$

$3 / 8=3 / 15$

$3 / 15=3 / 22$

$3 / 22-3 / 29$

$3 / 29-4 / 5$

$4 / 5-4 / 12$

$9 / 6-9 / 13$

$9 / 13=9 / 20$

$9 / 20-9 / 27$
4.29

3.98

3.88

4.63

4.81

4.51

1.17

1.65

2.77
$9 / 27=10 / 4$

$10 / 4-10 / 11$

$10 / 11-10 / 18$

$10 / 18-10 / 25$

$10 / 25-11 / 1$

$11 / 8-11 / 22$

$11 / 22-12 / 13$

$12 / 13=12 / 20$
2.26

2.09

1.90

1.67

2.55

2.16

2.02

1.68 
APPENDIX H

Table 2 ( continued)

Measurement Exposure Rate Measurement Period $\mathrm{mR} /$ Day
Exposure Rate $\mathrm{mR} /$ Day
Measurement Period
Exposure Rate • $\mathrm{mR} /$ Day

\section{Area}

$\begin{array}{ll}12 / 28 / 65-1 / 4 / 66 & 3.42 \\ 1 / 4-1 / 11 & 3.95 \\ 1 / 11-1 / 18 & 6.71 \\ 1 / 18-1 / 25 & 2.68 \\ 1 / 25=2 / 1 & 2.96 \\ 2 / 1-2 / 10 & 3.73 \\ 2 / 10=2 / 17 & 3.17 \\ 2 / 17-2 / 22 & 1.81 \\ 2 / 22=3 / 2 & 3.22 \\ 3 / 2=3 / 8 & 3.93 \\ 3 / 8=3 / 15 & 2.29 \\ 3 / 17=3 / 22 & 1.30 \\ 3 / 22=3 / 29 & 2.09 \\ 3 / 29=4 / 5 & 2.58 \\ 4 / 5-4 / 12 & 2.45 \\ 4 / 12=4 / 19 & \text { Lost } \\ 4 / 19=4 / 26 & 2.97\end{array}$

$4 / 26-5 / 3$

$5 / 3-5 / 10$

$5 / 10-5 / 17$

$5 / 17-5 / 24$

$5 / 24-5 / 31$

$5 / 31-6 / 7$

$6 / 7-6 / 14$

$6 / 14-6 / 21$

$6 / 21-6 / 28$

$6 / 28-7 / 5$

$7 / 5-7 / 15$

$7 / 15-7 / 19$

$7 / 19-7 / 26$

$7 / 26-8 / 1$

$8 / 1-8 / 10$

$8 / 10-8 / 16$
3.29

Off Scale

3.92

off Scale

3.16

1.61

1.12

1.55

1.91

1.67

1.18

0.78

0.49

0.61

3.21

0.53
$8 / 29-9 / 6$

$9 / 6-9 / 12$

$9 / 12-9 / 19$

$9 / 19-9 / 27$

$9 / 27-10 / 4$

$10 / 4-10 / 11$

$10 / 11-10 / 18$

$10 / 18=10 / 25$

$10 / 25-11 / 8$

$11 / 8-11 / 15$

$11 / 15-11 / 22$

$11 / 22-11 / 29$

$11 / 29-12 / 6$

$12 / 6=12 / 13$

$12 / 13=12 / 20$

$12 / 20-12 / 28$
0.16

1.59

1.93

2.66

1.88

2.26

2.21

1.87

3.09

2.61

3.01

2.83

2.20

2.95

2.79

3.85

Richland Marina

$\begin{array}{ll}12 / 28 / 65 \times 1 / 4 / 66 & 6.97 \\ 1 / 4=1 / 11 & 1.96 \\ 1 / 11=1 / 18 & 5.20 \\ 1 / 18=1 / 25 & 2.12 \\ 1 / 25-2 / 1 & 2.33 \\ 2 / 1=2 / 10 & 3.39 \\ 2 / 10=2 / 17 & 2.23 \\ 2 / 17=2 / 22 & 1.31 \\ 2 / 22=3 / 1 & 1.39 \\ 3 / 1=3 / 8 & 1.81 \\ 3 / 8=3 / 15 & 2.40 \\ 3 / 17=3 / 22 & 2.23 \\ 3 / 22=3 / 29 & 2.27 \\ 3 / 29=4 / 5 & 2.80\end{array}$

$\begin{array}{ll}4 / 5=4 / 12 & 2.99 \\ 4 / 12-4 / 19 & 2.09 \\ 4 / 19-4 / 26 & 1.68 \\ 4 / 26=5 / 3 & 1.16 \\ 5 / 3=5 / 10 & 6.31 \\ 5 / 10-5 / 17 & 2.58 \\ 5 / 17=5 / 24 & 2.36 \\ 5 / 24=5 / 31 & 2.08 \\ 5 / 31-6 / 7 & 1.34 \\ 6 / 7-6 / 14 & 0.55 \\ 6 / 14-6 / 21 & 1.12 \\ 6 / 21-6 / 28 & 1.61 \\ 6 / 28-7 / 5 & 1.09 \\ 9 / 6-9 / 16 & 0.96\end{array}$

$9 / 16-9 / 23$

1.23

$9 / 23-9 / 27$

1.61

$9 / 27-10 / 4$

$10 / 4=10 / 10$

0.86

$10 / 10=10 / 18$

1.60

$10 / 18-10 / 25$

1.58

$10 / 25$ - $11 / 8$

2.76

$11 / 8-11 / 15$

2.23

$11 / 15-11 / 22$

1.72

$11 / 22-11 / 29$

1.99

$11 / 29-12 / 6$

$12 / 6=12 / 13$

$12 / 13-12 / 20$

1.16

1.58

2.38

1.92

$12 / 20-12 / 28$

2.32

McNary Dam

$\begin{array}{ll}7 / 22-7 / 29 & 0.81 \\ 7 / 29=8 / 5 & 0.85 \\ 8 / 5-8 / 12 & 0.69 \\ 8 / 12=8 / 19 & 2.26 \\ 9 / 2=9 / 9 & 0.71 \\ 9 / 9-9 / 16 & 5.48\end{array}$

$9 / 16-9 / 23$

3.19

0.94

$9 / 23-9 / 30$

$9 / 30-10 / 7$

2.05

$10 / 7-10 / 14$

$10 / 14-10 / 21$

1.32

0.44

$10 / 21-10 / 28$

0.57

$10 / 28-11 / 11$

0.37

$11 / 11-11 / 18$

0.42

$11 / 18=11 / 23$

0.52

$11 / 23-12 / 16$

0.62

$12 / 16-12 / 22$

0.81

$12 / 22-12 / 31$

0.62 


\title{
DISTRIBUTION
}

\author{
Number of \\ Copies \\ Atomic Energy Commission, Washington \\ Division of Operational Safety \\ G. M. Dunning \\ A. A. Schoen \\ 2 \\ Douglas United Nuclear, Inc. \\ C. D. Corbit \\ R. G. Geier \\ 2 \\ Isochem, Inc. \\ G. E. Backman \\ D. J. Brown \\ $\underline{\text { ITT/Federal Support Services }}$ \\ R. H. Wilson \\ 1 \\ Oregon State Board of Health \\ Portland, Oregon 97201 \\ K. H. Spies \\ Oregon State Universtiy \\ Department of Oceanography \\ Corvallis, Oregon 97331 \\ C. L. Osterberg \\ 5 \\ Richland Operations Office \\ R. K. Sharp \\ R. B. St. John \\ M. W. Tiernan \\ C. N. Zangar \\ Technical Information Library \\ 1 U.S. Public Health Service \\ Radiation Surveillance Center \\ Washington, D.C. \\ 2 \\ University of Washington \\ Laboratory of Radiation Biology \\ Seattle, Washington 98105 \\ L. R. Donaldson \\ A. H. Seymour
}


Number of

Copies

1

Washington State Department of Health 1510 Smith Tower

Seattle, Washington 98104

R. L. Stockman

33

Battelle-Northwest

J. R. Boving don

L. A. Carter

J. P. Corley

T. H. Essig (13)

R. F. Foster

R. B. Hall

J. F. Honstead

W. C. Horton

R. L. Junkins

A. R. Keene

R. E. Nakatani

H. M. Parker

C. F. Smith

J. K. Soldat

Technical Publications (2)

Technical Information Files (5) 